University of Nebraska - Lincoln

DigitalCommons@University of Nebraska - Lincoln

Faculty Publications, Department of

Mathematics

Mathematics, Department of

January 2000

\title{
Boundary Controllability of Thermoelastic Plates via the Free Boundary Conditions
}

\author{
George Avalos \\ University of Nebraska-Lincoln, gavalos@math.unl.edu \\ Irena Lasiecka \\ University of Virginia
}

Follow this and additional works at: https://digitalcommons.unl.edu/mathfacpub

Part of the Mathematics Commons

Avalos, George and Lasiecka, Irena, "Boundary Controllability of Thermoelastic Plates via the Free Boundary Conditions" (2000). Faculty Publications, Department of Mathematics. 1.

https://digitalcommons.unl.edu/mathfacpub/1

This Article is brought to you for free and open access by the Mathematics, Department of at DigitalCommons@University of Nebraska - Lincoln. It has been accepted for inclusion in Faculty Publications, Department of Mathematics by an authorized administrator of DigitalCommons@University of Nebraska - Lincoln. 


\title{
BOUNDARY CONTROLLABILITY OF THERMOELASTIC PLATES VIA THE FREE BOUNDARY CONDITIONS*
}

\author{
GEORGE AVALOS ${ }^{\dagger}$ AND IRENA LASIECKA ${ }^{\ddagger}$
}

\begin{abstract}
Controllability properties of a partial differential equation (PDE) model describing a thermoelastic plate are studied. The PDE is composed of a Kirchoff plate equation coupled to a heat equation on a bounded domain, with the coupling taking place on the interior and boundary of the domain. The coupling in this PDE is parameterized by $\alpha>0$. Boundary control is exerted through the (two) free boundary conditions of the plate equation and through the Robin boundary condition of the temperature. These controls have the physical interpretation of inserted forces and moments and prescribed temperature, respectively, all of which act on the edges of the plate. The main result here is that under such boundary control, and with initial data in the basic space of well-posedness, one can simultaneously control the displacement of the plate exactly and the temperature approximately. Moreover, the thermal control may be taken to be arbitrarily smooth in time and space, and the thermal control region may be any nonempty subset of the boundary. This controllability holds for arbitrary values of the coupling parameter $\alpha$, with the optimal controllability time in line with that seen for uncoupled Kirchoff plates.
\end{abstract}

Key words. partial differential equations, exact-approximate controllability

AMS subject classification. 35B37

PII. S0363012998339836

\section{Introduction.}

1.1. Statement of the problem. Throughout, $\Omega$ will be a bounded open subset of $\mathbb{R}^{2}$ with sufficiently smooth boundary $\Gamma=\overline{\Gamma_{0}} \cup \overline{\Gamma_{1}}$, with both $\Gamma_{0}$ and $\Gamma_{1}$ being open, with $\Gamma_{0}$ being possibly empty, and satisfying $\overline{\Gamma_{0}} \cap \overline{\Gamma_{1}}=\varnothing$. Furthermore, $\Gamma_{2}$ will be any open and nonempty subset of $\Gamma_{1}$. With this geometry, we shall consider here the following thermoelastic system on finite time $(0, T)$ :

$$
\left\{\begin{array}{l}
\left\{\begin{array}{l}
\omega_{t t}-\gamma \Delta \omega_{t t}+\Delta^{2} \omega+\alpha \Delta \theta=0 \\
\beta \theta_{t}-\eta \Delta \theta+\sigma \theta-\alpha \Delta \omega_{t}=0
\end{array} \text { on }(0, T) \times \Omega ;\right. \\
\omega=\frac{\partial \omega}{\partial \nu}=0 \text { on }(0, T) \times \Gamma_{0} ; \\
\left\{\begin{array}{l}
\Delta \omega+(1-\mu) B_{1} \omega+\alpha \theta=u_{1} \\
\frac{\partial \Delta \omega}{\partial \nu}+(1-\mu) \frac{\partial B_{2} \omega}{\partial \tau}-\gamma \frac{\partial \omega_{t t}}{\partial \nu}+\alpha \frac{\partial \theta}{\partial \nu}=u_{2}
\end{array} \text { on }(0, T) \times \Gamma_{1} ;\right. \\
\frac{\partial \theta}{\partial \nu}+\lambda \theta=\left\{\begin{array}{cc}
u_{3} & \text { on }(0, T) \times \Gamma_{2}, \\
0 & \text { on }(0, T) \times \Gamma \backslash \Gamma_{2},
\end{array} \quad \lambda \geq 0 ;\right. \\
\omega(t=0)=\omega_{0}, \omega_{t}(t=0)=\omega_{1}, \theta(t=0)=\theta_{0} \quad \text { on } \Omega .
\end{array}\right.
$$

* Received by the editors June 5, 1998; accepted for publication (in revised form) April 27, 1999; published electronically January 11, 2000.

http://www.siam.org/journals/sicon/38-2/33983.html

${ }^{\dagger}$ Department of Mathematics and Statistics, Texas Tech University, Lubbock, TX 79409 (avalos@math.ttu.edu). The research of this author was partially supported by NSF grant DMS9710981.

${ }^{\ddagger}$ Department of Mathematics, Thornton Hall, University of Virginia, Charlottesville, VA 22903 (il2v@amsun.apma.virginia.edu). The research of this author was partially supported by NSF grant DMS-9804822 and Army Research Office grant DAAH04-98-1-0059. 
Here, $\alpha, \beta, \eta$, and $\sigma$ are positive constants. The positive constant $\gamma$ is proportional to the thickness of the plate and assumed to be small with $0<\gamma \leq M$. The boundary operators $B_{i}$ are given by

$B_{1} \omega \equiv 2 \nu_{1} \nu_{2} \frac{\partial^{2} \omega}{\partial x \partial y}-\nu_{1}^{2} \frac{\partial^{2} \omega}{\partial y^{2}}-\nu_{2}^{2} \frac{\partial^{2} \omega}{\partial x^{2}}$ and $B_{2} \omega \equiv\left(\nu_{1}^{2}-\nu_{2}^{2}\right) \frac{\partial^{2} \omega}{\partial x \partial y}+\nu_{1} \nu_{2}\left(\frac{\partial^{2} \omega}{\partial y^{2}}-\frac{\partial^{2} \omega}{\partial x^{2}}\right)$.

The constant $\mu \in\left(0, \frac{1}{2}\right)$ is the familiar Poisson ratio, and $\nu=\left[\nu_{1}, \nu_{2}\right]$ denotes the outward unit normal to the boundary. Here and throughout we shall make the following geometric assumption on the (uncontrolled) portion of the boundary $\Gamma_{0}$ :

$$
\text { with } \bar{h}(x, y) \equiv\left[x-x_{0}, y-y_{0}\right], \exists\left\{x_{0}, y_{0}\right\} \in \mathbb{R}^{2} \text { such that } \bar{h}(x, y) \cdot \nu \leq 0 \text { on } \Gamma_{0} \text {. }
$$

The PDE model (1.1), with boundary functions $u_{1}=u_{2}=0$ and $u_{3}=0$, mathematically describes an uncontrolled Kirchoff plate subjected to a thermal damping, with the displacement of the plate represented by the function $\omega(t, x, y)$ and the temperature given by the function $\theta(t, x, y)$ (see [11] for a derivation of this model). The given control variables $u_{1}(t, x)$ and $u_{2}(t, x)$ are defined on the portion of the boundary $(0, T) \times \Gamma_{1}$; the control $u_{3}(t, x)$ is defined on $(0, T) \times \Gamma_{2}$.

Making the denotation

$$
H_{\Gamma_{0}}^{k}(\Omega) \equiv\left\{\varpi \in H^{k}(\Omega):\left.\frac{\partial^{j} \varpi}{\partial \nu^{j}}\right|_{\Gamma_{0}}=0 \text { for } j=0, \ldots, k-1\right\},
$$

we will throughout take the initial data $\left[\omega_{0}, \omega_{1}, \theta_{0}\right]$ to be in $H_{\Gamma_{0}}^{2}(\Omega) \times H_{\Gamma_{0}}^{1}(\Omega) \times L^{2}(\Omega)$. For initial data in these spaces and controls $u_{1}=u_{2}=0$ and $u_{3}=0$, one can show the well-posedness of (1.1) with the corresponding solution $\left[\omega, \omega_{t}, \theta\right]$ being in $C\left([0, T] ; H_{\Gamma_{0}}^{2}(\Omega) \times H_{\Gamma_{0}}^{1}(\Omega) \times L^{2}(\Omega)\right)$ (see, e.g., [11] and [2]). In this paper, we will study controllability properties of solutions of (1.1) under the influence of boundary control functions in the following spaces:

$\left[u_{1}, u_{2}, u_{3}\right] \in L^{2}\left(0, T ; L^{2}\left(\Gamma_{1}\right) \times H^{-1}\left(\Gamma_{1}\right)\right) \times C^{r}\left(\Sigma_{2, T}\right)$, where $r>0$ and $\Sigma_{2, T}=(0, T) \times \Gamma_{2}$.

For arbitrary $\left[u_{1}, u_{2}, u_{3}\right]$ of such smoothness, the corresponding solution $\left[\omega, \omega_{t}, \theta\right]$ will be in the "large" space $C\left([0, T] ;\left[D\left(\mathcal{A}_{\gamma}^{*}\right)\right]^{\prime}\right)$ (see the definition of $D\left(\mathcal{A}_{\gamma}^{*}\right)$ in $(1.49)$ ). In particular, we intend to address, on the finite time interval $[0, T]$, the question of exact-approximate controllability (this term being originally coined in [6]). That is to say, for given data $\left[\omega_{0}, \omega_{1}, \theta_{0}\right]$ (initial) and $\left[\omega_{0}^{T}, \omega_{1}^{T}, \theta_{0}^{T}\right]$ (terminal) in $H_{\Gamma_{0}}^{2}(\Omega) \times$ $H_{\Gamma_{0}}^{1}(\Omega) \times L^{2}(\Omega)$, and arbitrary $\epsilon>0$, is there a suitable control triple $\left[u_{1}, u_{2}, u_{3}\right] \epsilon$ $L^{2}\left(0, T ; L^{2}\left(\Gamma_{1}\right) \times H^{-1}\left(\Gamma_{1}\right)\right) \times C^{r}\left(\Sigma_{2, T}\right)$ such that the corresponding solution $\left[\omega, \omega_{t}, \theta\right]$ of (1.1) satisfies the following steering property at terminal time $T$ :

$$
\left[\omega(T), \omega_{t}(T)\right]=\left[\omega_{0}^{T}, \omega_{1}^{T}\right] \text { and }\left\|\theta(T)-\theta_{0}^{T}\right\|_{L^{2}(\Omega)} \leq \epsilon ?
$$

In this regard, we post our main result here for which we need the number

$$
T^{*} \equiv 2 \sqrt{\gamma} \cdot \max \left\{\sqrt{\frac{2}{1-\mu}} \max _{[x, y] \in \bar{\Omega}}|\bar{h}(x, y)|, \sup _{[x, y] \in \Omega} d\left([x, y], \Gamma_{2}\right)\right\},
$$

where, above, $d\left([x, y], \Gamma_{2}\right)$ denotes the distance between $[x, y]$ and $\Gamma_{2}$. 
TheOREM 1.1. Let assumptions (1.3) and (1.6) stand. Then for $T>T^{*}$, the following controllability property holds true: For given initial data $\left[\omega_{0}, \omega_{1}, \theta_{0}\right]$ and terminal data $\left[\omega_{0}^{T}, \omega_{1}^{T}, \theta_{0}^{T}\right]$ in the space $H_{\Gamma_{0}}^{2}(\Omega) \times H_{\Gamma_{0}}^{1}(\Omega) \times L^{2}(\Omega)$, and arbitrary $\epsilon>0$, one can find control functions $\left[u_{1}^{*}, u_{2}^{*}, u_{3}^{*}\right] \in L^{2}\left(0, T ; L^{2}\left(\Gamma_{1}\right) \times H^{-1}\left(\Gamma_{1}\right)\right) \times$ $C^{r}\left(\Sigma_{2, T}\right)$ (where arbitrary $r \geq 0$ ) such that the corresponding solution $\left[\omega^{*}, \omega_{t}^{*}, \theta^{*}\right]$ to (1.1) satisfies at terminal time $T$,

$$
\begin{gathered}
{\left[\omega^{*}(T), \omega_{t}^{*}(T)\right]=\left[\omega_{0}^{T}, \omega_{1}^{T}\right]} \\
\left\|\theta^{*}(T)-\theta_{0}^{T}\right\|_{L^{2}(\Omega)}<\epsilon
\end{gathered}
$$

Theorem 1.1 is almost a corollary from the following controllability result for the mechanical variable only, which comprises the bulk of our effort here.

THEOREM 1.2. With the coupling parameter $\alpha$ in (1.1) being arbitrary, and (1.3), (1.6) in place, then for $T>T^{*}$, the following property holds true: For all initial data $\left[\omega_{0}, \omega_{1}, \theta_{0}\right] \in H_{\Gamma_{0}}^{2}(\Omega) \times H_{\Gamma_{0}}^{1}(\Omega) \times L^{2}(\Omega)$ and terminal data $\left[\omega_{0}^{T}, \omega_{1}^{T}\right] \in$ $H_{\Gamma_{0}}^{2}(\Omega) \times H_{\Gamma_{0}}^{1}(\Omega)$, there exists $\left[u_{1}, u_{2}, u_{3}\right] \in L^{2}\left(0, T ; L^{2}\left(\Gamma_{1}\right) \times H^{-1}\left(\Gamma_{1}\right)\right) \times H^{s}\left(\Sigma_{2, T}\right)$, where arbitrary $s \geq 0$, such that the corresponding solution $\left[\omega, \omega_{t}, \theta\right]$ to (1.1) satisfies $\left[\omega(T), \omega_{t}(T)\right]=\left[\omega_{0}^{T}, \omega_{1}^{T}\right]$.

Remark 1.3. Note that the point $\left[x_{0}, y_{0}\right]$ can be selected in such a way so that $2 \max _{[x, y] \in \bar{\Omega}}|\bar{h}(x, y)| \leq \operatorname{diam}(\Omega)$, and so, ultimately, $T^{*}$ in (1.6) can be rechosen as $T^{*}=2 \sqrt{\gamma} \operatorname{diam}(\Omega)$.

Remark 1.4. Note that in our statement of controllability, no geometric conditions are imposed on the controlled region of the boundary $\Gamma_{1}$, only on the (possibly void) boundary portion $\Gamma_{0}$.

1.2. Literature. To date, the only work dealing with the boundary control of thermoelastic plates, in dimension greater than one, had been that of J. Lagnese in [12] (indeed, this present paper is principally motivated by [12]). In this paper, Lagnese shows that if the coupling parameter $\alpha$ is small enough and the boundary $\Gamma$ is "star shaped," then the boundary controlled system (1.1) is (partially) exactly controllable with respect to the mechanical variables $\left[\omega, \omega_{t}\right]$. Also in [22], a boundary-controlled system of thermoelastic waves is studied, with a coupling parameter $\alpha$ likewise present therein, and a result of partial exact controllability for this PDE is cited (again, controllability with respect to the hyperbolic component). This controllability result is quoted in [22] to be valid for all sizes of $\alpha$; however, in the erratum [23], the author of [22] has acknowledged a flaw in the controllability proof, the correction of which will necessitate a smallness criterion on $\alpha$. Ultimately, then, the paper [22] produces a controllability result if the coupling parameter is small enough, a result in the style of [12]. The chief contribution of the present paper is to remove restrictions on the size of $\alpha$ (see Theorem 1.2 above). For a one-dimensional version of (1.1), S. Hansen and B. Zhang in [8], via a moment problem approach, show the system's exact null controllability with boundary control in either the plate or the thermal component.

Other controllability results for the thermoelastic system, which do not assume any "smallness" condition on the coupling parameters, involve the implementation of distributed/internal controls subject to clamped or hinged boundary conditions. These results include that in [6], in which interior control is placed in the Kirchoff plate component subject to clamped boundary conditions. 
With such control, one obtains exact controllability for the plate $\left[\omega, \omega_{t}\right]$ and approximate controllability for the temperature $\theta$ (i.e., exact-approximate controllability). In addition, the work in [19] deals with obtaining a result of null controllability for a linear system of thermoelasticity, in which both the hyperbolic and the parabolic components can be driven to zero by means of interior control placed in the hyperbolic (wave) component.

Another result of internal control for the thermoelastic PDE (1.1) is in [5], wherein interior control is placed in the heat equation only (i.e., $\beta \theta_{t}-\eta \Delta \theta+\sigma \theta-\alpha \Delta \omega_{t}=u$ ) so as to obtain exact controllability for both components $\omega$ and $\theta$. The novelty of this result is that this (total) exact controllability obtains for all values of the rotational inertia parameter $\gamma \geq 0$ : in the limiting case $\gamma=0$, one is then presented with a result of exact controllability for a PDE modeled by the generator of an analytic semigroup (see [18]). This controllability holds for all values of $\alpha$.

Again, the main contribution of this paper is that we consider boundary controls acting via the higher order free mechanical boundary conditions, and we do not assume any size restriction on the coupling parameter $\alpha$. Moreover, we do not impose any geometric "star-shaped" conditions on the controlled portion of the geometry.

At this point, we attempt to compare the degree of difficulty in obtaining controllability results for thermoelastic plates under mechanical interior control with lowerorder mechanical boundary conditions enforced (such as clamped or hinged), versus that involved in the present study, where, again, boundary control is exerted upon the second and third order free boundary conditions. This comparison is appropriate, since the novelty of our work is touted to be (mechanical) exact controllability for the PDE (1.1), whatever $\alpha$ may be; and excluding the paper [5], the only other available controllability results for thermoelastic systems, which require no size constraints on $\alpha$, concerned thermoelastic systems under (distributed) interior mechanical control and with lower mechanical boundary conditions in place.

An underlying strategy in control theoretic studies of thermoelastic plates has been to exploit, if possible, previously known controllability results for (uncoupled) Kirchoff plates. To this end, one attempts to treat the thermoelastic system as a sort of perturbation of the Kirchoff plate. It is well known that if the underlying controllability map can be decomposed into the sum of a compact map and a surjective controllability map, corresponding to a (simpler) subcomponent of the PDE system, then the exact controllability of the original problem is equivalent to its approximate controllability. This favorable scenario occurs in equations of thermoelasticity with either clamped or hinged boundary conditions and interior, distributed controls (see, e.g., [20]). Indeed, the part of the simpler component is played by the classical and much-studied Kirchoff plate, for which many results on exact controllability are already available in the literature. Taking the boundary conditions to be clamped or hinged allows for a known structural decomposition of the thermoelastic system into a group (associated with the Kirchoff plate) and a compact perturbation. Combining this decomposition with the boundedness of interior control actions immediately yields the desired decomposition of the original controllability map into the sum of a surjective controllability map (corresponding to the Kirchoff plate) and a compact perturbation. This popular strategy was used in [6], where an exact-approximate controllability result was established for the thermoelastic system with clamped homogeneous boundary conditions and internal controls.

The situation is drastically different in the present paper, involving the case of boundary controls. Here, in this case of free mechanical boundary conditions, the 
corresponding controllability operator cannot be taken to be a compact perturbation of the controllability map for the (uncoupled) boundary-controlled Kirchoff plate. In the first place, the associated input $\rightarrow$ state space map, defined explicitly in (1.42), is an inherently unbounded operator with respect to the natural energy space (see [17] for recent sharp regularity results for corresponding solutions, which are still, however, below the level of energy). Moreover, in the present case of free boundary conditions, there is a decomposition of the underlying thermoelastic semigroup, but it is into the sum of a Kirchoff plate semigroup and an unbounded - not compact - operator (see [16]). This complication is due to the fact that the Lopatinski conditions are not satisfied for the Kirchoff model under free boundary conditions, and to the intrinsic nature of the coupling between the mechanical and thermal variables within the free boundary conditions. These two complications above, again an artifact of the "free case," explain why there have been so few results regarding the boundary control of thermoelastic plates and why a "decoupling" of the thermoelastic PDE into a sole Kirchoff plate can only go so far.

Our goal here is to dispense with this smallness assumption and, in addition, show that a control can be constructed that provides exact controllability of the mechanical variables and approximate controllability of the thermal component. We note that the thermal control $u_{3}$ present in (1.1) — wholly absent in [12] — plays no part at all in the removal of the size restriction on $\alpha$; it is in place only to exploit, in a compactness-uniqueness argument, recently obtained approximate controllability properties of the thermoelastic plate under the action of boundary control in the free mechanical boundary conditions (see [10]). At this point in time, the thermoelastic system cannot be shown to be approximately controllable with control in the free boundary conditions only (and no thermal control). Therefore, the presence of the thermal boundary control here is not an artificiality; it appears to be necessary for approximate controllability. (We do not know if the future will bring a unique continuation result for the thermoelastic plate in the absence of the thermal component.) However, the result of Theorem 1.1 says that the thermal control may be taken to be very smooth and with arbitrarily small support $\Gamma_{2}$. Again, this benign situation is a consequence of our employing thermal control at the compactness-uniqueness level only; it plays no part whatsoever in generating the main observability estimate (estimate (2.5) of Theorem 2.1), this being free of any size restrictions on $\alpha$.

The strategy adopted in this paper consists of the following steps. Initially, a suitable transformation of variables is made and applied to (1.1); subsequently, a multiplier method is invoked with respect to the transformed equation. The mulitiplers employed here are the differential multipliers used in the study of exact controllability for the Kirchoff plate model (inspired by [11]), together with the nonlocal ( $\Psi D O$ ) multipliers used in the study of thermoelastic plates in [3] and [4]. The controllability time $T^{*}$ in Theorem 1.1 ultimately depends in part upon the radial vector field associated with the differential Kirchoff multipliers (see Lemma 2.5 below). This multiplier method allows the attainment of preliminary estimates for the energy of the system. However, these estimates are "polluted" by certain boundary terms that are not majorized by the energy. To cope with these, we use the sharp trace estimates established in [15] for Kirchoff plates. The use of this PDE result introduces lower order terms into the energy estimate, which are eventually eliminated with the help of a new unique continuation result in [10]. It is only at the level of invoking this uniqueness result that the thermal control $u_{3}$ on $\Gamma_{2}$ must be introduced. The controllability time $T^{*}$ in (1.6) is optimal. 


\subsection{Operator theoretic formulation and analysis.}

1.3.1. Preliminary definitions. In obtaining our controllability result Theorem 1.1, it will be useful to consider the PDE system (1.1) as an abstract evolution equation in a certain Hilbert space, to which end we introduce the following definitions and notation.

- With $H_{\Gamma_{0}}^{k}(\Omega)$ as defined in (1.4), we define $\AA$ : $L^{2}(\Omega) \supset D(\AA) \rightarrow L^{2}(\Omega)$ to be $\AA=\Delta^{2}$, with domain

$$
\begin{array}{r}
D(\AA)=\left\{\omega \in H^{4}(\Omega) \cap H_{\Gamma_{0}}^{2}(\Omega): \Delta \omega+(1-\mu) B_{1} \omega=0 \text { on } \Gamma_{1}\right. \text { and } \\
\left.\frac{\partial \Delta \omega}{\partial \nu}+(1-\mu) \frac{\partial B_{2} \omega}{\partial \tau}=0 \text { on } \Gamma_{1}\right\} .
\end{array}
$$

- $\AA$ is then positive definite and self-adjoint, and consequently from [7] we have the characterizations

$$
\begin{aligned}
& D\left(\AA^{\frac{1}{4}}\right)=H_{\Gamma_{0}}^{1}(\Omega), \\
& D\left(\AA^{\frac{1}{2}}\right)=H_{\Gamma_{0}}^{2}(\Omega), \\
& D\left(\AA^{\frac{3}{4}}\right)=\left\{\omega \in H^{3}(\Omega) \cap H_{\Gamma_{0}}^{2}(\Omega): \Delta \omega+(1-\mu) B_{1} \omega=0 \text { on } \Gamma_{1}\right\} .
\end{aligned}
$$

Note that without loss of generality, we are here taking $\Gamma_{0}$ to be nonempty in order to have the equivalence of the $H^{2}(\Omega)$ norm with that induced by the $D\left(\AA^{\frac{1}{2}}\right)$. In the case that $\Gamma_{0}=\emptyset$, we would simply modify $D(\AA)$ by enforcing $\frac{\partial \Delta \omega}{\partial \nu}+\left.(1-\mu) \frac{\partial B_{2} \omega}{\partial \tau}\right|_{\Gamma_{1}}=\left.\omega\right|_{\Gamma_{1}}$ (instead of $\frac{\partial \Delta \omega}{\partial \nu}+\left.(1-\mu) \frac{\partial B_{2} \omega}{\partial \tau}\right|_{\Gamma_{1}}=0$ in (1.7)). This modification would not change the problem.

Moreover, using Green's formula in [11], we have that for $\omega, \widehat{\omega}$ "smooth enough,"

$$
\begin{aligned}
\int_{\Omega}\left(\Delta^{2} \omega\right) \widehat{\omega} d \Omega= & a(\omega, \widehat{\omega})+\int_{\Gamma}\left[\frac{\partial \Delta \omega}{\partial \nu}+(1-\mu) \frac{\partial B_{2} \omega}{\partial \tau}\right] \widehat{\omega} d \Gamma \\
& -\int_{\Gamma}\left[\Delta \omega+(1-\mu) B_{1} \omega\right] \frac{\partial \widehat{\omega}}{\partial \nu} d \Gamma
\end{aligned}
$$

where $a(\cdot, \cdot)$ is defined by

$a(\omega, \widehat{\omega}) \equiv \int_{\Omega}\left[\omega_{x x} \widehat{\omega}_{x x}+\omega_{y y} \widehat{\omega}_{y y}+\mu\left(\omega_{x x} \widehat{\omega}_{y y}+\omega_{y y} \widehat{\omega}_{x x}\right)+2(1-\mu) \omega_{x y} \widehat{\omega}_{x y}\right] d \Omega$.

In particular, this formula and the second characterization in (1.8) give that for all $\omega, \widehat{\omega} \in D\left(\AA^{\frac{1}{2}}\right)$,

$$
\begin{array}{r}
\langle\AA \omega, \widehat{\omega}\rangle_{\left[D\left(\AA^{\frac{1}{2}}\right)\right]^{\prime} \times D\left(\AA^{\frac{1}{2}}\right)}=\left(\AA^{\frac{1}{2}} \omega, \AA^{\frac{1}{2}} \widehat{\omega}\right)_{L^{2}(\Omega)}=a(\omega, \widehat{\omega})_{L^{2}(\Omega)}, \\
\|\omega\|_{D\left(\AA^{\frac{1}{2}}\right)}^{2}=\left\|\AA^{\frac{1}{2}} \omega\right\|_{L^{2}(\Omega)}^{2}=a(\omega, \omega) .
\end{array}
$$

- We define $A_{D}: L^{2}(\Omega) \supset D\left(A_{D}\right) \rightarrow L^{2}(\Omega)$ to be $A_{D}=-\Delta$, with Dirichlet boundary conditions, viz.,

$$
D\left(A_{D}\right)=H^{2}(\Omega) \cap H_{0}^{1}(\Omega) .
$$


$A_{D}$ is also positive definite, self-adjoint, and by [7]

$$
D\left(A_{D}^{\frac{1}{2}}\right)=H_{0}^{1}(\Omega)
$$

- We denote the operator $A_{R}: L^{2}(\Omega) \supset D\left(A_{R}\right) \rightarrow L^{2}(\Omega)$ by the following second order elliptic operator:

$$
A_{R}=-\Delta+\frac{\sigma}{\eta} \mathbf{I}, \text { with } D\left(A_{R}\right)=\left\{\vartheta \in H^{2}(\Omega): \frac{\partial \vartheta}{\partial \nu}+\lambda \vartheta=0\right\}
$$

$A_{R}$ is self-adjoint, positive definite on $L^{2}(\Omega)$, with its fractional powers therefore being well defined. In particular, we have again by [7] that for $s \in\left[0, \frac{3}{4}\right)$,

$$
\begin{aligned}
& D\left(A_{R}^{s}\right)=H^{2 s}(\Omega) \\
& (\vartheta, \widetilde{\vartheta})_{H^{1}(\Omega)}=\left(A_{R}^{\frac{1}{2}} \vartheta, A_{R}^{\frac{1}{2}} \widetilde{\vartheta}\right)_{L^{2}(\Omega)} \\
& =(\nabla \vartheta, \nabla \widetilde{\vartheta})_{L^{2}(\Omega)}+\lambda(\vartheta, \widetilde{\vartheta})_{L^{2}(\Gamma)}+\frac{\sigma}{\eta}(\vartheta, \widetilde{\vartheta})_{L^{2}(\Omega)} .
\end{aligned}
$$

- We denote the operator $A_{N}: L^{2}(\Omega) \supset D\left(A_{N}\right) \rightarrow L^{2}(\Omega)$ by the following second order elliptic operator:

$$
A_{N}=-\Delta, \text { with } D\left(A_{N}\right)=\left\{\vartheta \in H^{2}(\Omega):\left.\vartheta\right|_{\Gamma_{0}}=\left.\frac{\partial \vartheta}{\partial \nu}\right|_{\Gamma_{1}}=0\right\}
$$

Once again by [7], we have for $s \in\left(\frac{1}{4}, \frac{3}{4}\right)$

$$
D\left(A_{N}^{s}\right)=\left\{\vartheta \in H^{2 s}(\Omega) \text { such that }\left.\vartheta\right|_{\Gamma_{0}}=0\right\} .
$$

- $\left(\gamma_{0}, \gamma_{1}\right)$ will denote the classical Sobolev trace maps, which yield for $f \in$ $C^{\infty}(\bar{\Omega})$

$$
\gamma_{0} f=\left.f\right|_{\Gamma} ; \gamma_{1} f=\left.\frac{\partial f}{\partial \nu}\right|_{\Gamma} .
$$

- We define the elliptic operators $G_{1}, G_{2}$, and $D$ as follows:

$$
G_{1} h=v \Longleftrightarrow\left\{\begin{array}{l}
\Delta^{2} v=0 \quad \text { on } \Omega, \\
v=\frac{\partial v}{\partial \nu}=0 \quad \text { on } \Gamma_{0}, \\
\left\{\begin{array}{l}
\Delta v+(1-\mu) B_{1} v=h \\
\frac{\partial \Delta v}{\partial \nu}+(1-\mu) \frac{\partial B_{2} v}{\partial \tau}=0
\end{array} \text { on } \Gamma_{1},\right.
\end{array}\right.
$$

$$
G_{2} h=v \Longleftrightarrow\left\{\begin{array}{l}
\Delta^{2} v=0 \quad \text { on } \Omega, \\
v=\frac{\partial v}{\partial \nu}=0 \quad \text { on } \Gamma_{0}, \\
\left\{\begin{array}{l}
\Delta v+(1-\mu) B_{1} v=0 \\
\frac{\partial \Delta v}{\partial \nu}+(1-\mu) \frac{\partial B_{2} v}{\partial \tau}=h
\end{array} \text { on } \Gamma_{1},\right.
\end{array}\right.
$$


$D h=v \Longleftrightarrow\left\{\begin{array}{cc}\Delta v=0 & \text { on } \Omega, \\ \left.v\right|_{\Gamma}=h & \text { on } \Gamma ;\end{array} \quad R h=v \Longleftrightarrow\left\{\begin{array}{l}\left(-\Delta+\frac{\sigma}{\eta} \mathbf{I}\right) v=0 \quad \text { on } \Omega, \\ \begin{cases}\frac{\partial v}{\partial \nu}+\lambda v=h & \text { on } \Gamma_{2}, \\ \frac{\partial v}{\partial \nu}+\lambda v=0 & \text { on } \Gamma \backslash \Gamma_{2} .\end{cases} \end{array}\right.\right.$

The classic regularity results of $[21$, p. 152] then provide that for all $q$ real,

$$
\left\{\begin{array}{l}
D \in \mathcal{L}\left(H^{q}(\Gamma), H^{q+\frac{1}{2}}(\Omega)\right) \\
R \in \mathcal{L}\left(H_{0}^{q}\left(\Gamma_{2}\right), H^{q+\frac{3}{2}}(\Omega)\right) \\
G_{1} \in \mathcal{L}\left(H_{0}^{q}\left(\Gamma_{1}\right), H^{q+\frac{5}{2}}(\Omega)\right) \\
G_{2} \in \mathcal{L}\left(H_{0}^{q-\frac{1}{2}}\left(\Gamma_{1}\right), H^{q+3}(\Omega)\right) .
\end{array}\right.
$$

Denoting the topological dual of $H^{q}$ as $\left[H^{q}\right]^{\prime}$ (pivotal with respect to the $L^{2}$-inner product), then with the elliptic operators $A_{R}$ and $R$ as defined above, one can show that for $q \geq-\frac{1}{2}$, the (Banach space) adjoint $R^{*} A_{R} \in$ $\mathcal{L}\left(D\left(A_{R}^{\frac{1}{2}}\right),\left[H^{q}\left(\Gamma_{2}\right)\right]^{\prime}\right)$ satisfies

$$
R^{*} A_{R} \vartheta=\left.\vartheta\right|_{\Gamma_{2}} \text { for all } \vartheta \in D\left(A_{R}^{\frac{1}{2}}\right) .
$$

Moreover, with the operators $\AA$ and $G_{i}$ as defined above, one can readily show with the use of Green's formula (1.9) that $\forall \varpi \in D\left(\AA^{\frac{1}{2}}\right)$ the (Banach space) adjoints $G_{i}^{*} \AA \in \mathcal{L}\left(D\left(\AA^{\frac{1}{2}}\right), H^{i-\frac{1}{2}}\left(\Gamma_{1}\right)\right)$ satisfy for $i=1,2$,

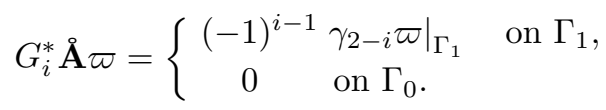

- With $A_{N}$ given by (1.16), we define the operator $P_{\gamma}: D\left(P_{\gamma}\right) \subset L^{2}(\Omega) \rightarrow$ $L^{2}(\Omega)$ by

$$
P_{\gamma} \equiv \mathbf{I}+\gamma A_{N}
$$

(i) With the parameter $\gamma>0$, we define a space $H_{\Gamma_{0}, \gamma}^{1}(\Omega)$ equivalent to $H_{\Gamma_{0}}^{1}(\Omega)$ with inner product

$$
\left(\omega_{1}, \omega_{2}\right)_{H_{\Gamma_{0}, \gamma}^{1}(\Omega)} \equiv\left(\omega_{1}, \omega_{2}\right)_{L^{2}(\Omega)}+\gamma\left(\nabla \omega_{1}, \nabla \omega_{2}\right)_{L^{2}(\Omega)} \quad \forall \omega_{1}, \omega_{2} \in H_{\Gamma_{0}}^{1}(\Omega)
$$

and with its dual denoted as $H_{\Gamma_{0}, \gamma}^{-1}(\Omega)$. After recalling that $H_{\Gamma_{0}}^{1}(\Omega)=D\left(A_{N}^{\frac{1}{2}}\right)$ (by (1.17)), two extensions by continuity will then yield that

$$
\begin{aligned}
& P_{\gamma} \in \mathcal{L}\left(H_{\Gamma_{0}, \gamma}^{1}(\Omega), H_{\Gamma_{0}, \gamma}^{-1}(\Omega)\right) \\
& \text { with }\left\langle P_{\gamma} \omega_{1}, \omega_{2}\right\rangle_{H_{\Gamma_{0}, \gamma}^{-1}(\Omega) \times H_{\Gamma_{0}, \gamma}^{1}(\Omega)}=\left(\omega_{1}, \omega_{2}\right)_{H_{\Gamma_{0}, \gamma}^{1}(\Omega)} .
\end{aligned}
$$

Furthermore, the obvious $H_{\Gamma_{0}, \gamma}^{1}(\Omega)$-ellipticity of $P_{\gamma}$ and Lax-Milgram give us that $P_{\gamma} \in \mathcal{L}\left(H_{\Gamma_{0}, \gamma}^{1}(\Omega), H_{\Gamma_{0}, \gamma}^{-1}(\Omega)\right)$ is boundedly invertible, with

$$
P_{\gamma}^{-1} \in \mathcal{L}\left(H_{\Gamma_{0}, \gamma}^{-1}(\Omega), H_{\Gamma_{0}, \gamma}^{1}(\Omega)\right) .
$$


Moreover, because $P_{\gamma}$ is positive definite and self-adjoint as an operator $P_{\gamma}$ : $L^{2}(\Omega) \supset D\left(P_{\gamma}\right) \rightarrow L^{2}(\Omega)$, the square root $P_{\gamma}^{\frac{1}{2}}$ is consequently well defined with $D\left(P_{\gamma}^{\frac{1}{2}}\right)=H_{\Gamma_{0}, \gamma}^{1}(\Omega)$, by (1.17). It then follows from (1.25) and (1.26) that for $\omega$ and $\widehat{\omega} \in H_{\Gamma_{0}, \gamma}^{1}(\Omega)$,

$$
\begin{aligned}
\left\|P_{\gamma}^{\frac{1}{2}} \omega\right\|_{L^{2}(\Omega)}^{2}=\|\omega\|_{L^{2}(\Omega)}^{2}+\gamma\|\nabla \omega\|_{L^{2}(\Omega)}^{2} & =\|\omega\|_{H_{\Gamma_{0}, \gamma}^{1}(\Omega)}^{2}, \\
\left(P_{\gamma}^{\frac{1}{2}} \omega, P_{\gamma}^{\frac{1}{2}} \widehat{\omega}\right)_{L^{2}(\Omega)} & =(\omega, \widehat{\omega})_{H_{\Gamma_{0}, \gamma}^{1}(\Omega)} .
\end{aligned}
$$

(ii) Finally, by Green's formula we have for $\omega, \widehat{\omega} \in D\left(\AA^{\frac{1}{2}}\right)$,

$$
\begin{gathered}
\gamma\left\langle\left(\Delta+\AA G_{2} \gamma_{1}\right) \omega, \widehat{\omega}\right\rangle_{H_{\Gamma_{0}, \gamma}^{-1}(\Omega) \times H_{\Gamma_{0}, \gamma}^{1}(\Omega)} \\
=-\gamma(\nabla \omega, \nabla \widehat{\omega})_{L^{2}(\Omega)}+\gamma\left(\frac{\partial \omega}{\partial \nu}, \widehat{\omega}\right)_{L^{2}\left(\Gamma_{1}\right)}+\gamma\left(\gamma_{1} \omega, G_{2}^{*} \AA \widehat{\omega}\right)_{L^{2}\left(\Gamma_{1}\right)} \\
(1.30)=-\gamma(\nabla \omega, \nabla \widehat{\omega})_{L^{2}(\Omega)}=-\gamma\left\langle A_{N} \omega, \widehat{\omega}\right\rangle_{H_{\Gamma_{0}, \gamma}^{-1}(\Omega) \times H_{\Gamma_{0}, \gamma}^{1}(\Omega)}
\end{gathered}
$$

after using (1.23). We thus obtain after two extensions by continuity to $H_{\Gamma_{0}, \gamma}^{1}(\Omega)$ that

(1.31) $P_{\gamma}=\mathbf{I}-\gamma\left(\Delta+\AA G_{2} \gamma_{1}\right)$ as elements of $\mathcal{L}\left(H_{\Gamma_{0}, \gamma}^{1}(\Omega), H_{\Gamma_{0}, \gamma}^{-1}(\Omega)\right)$.

In obtaining the equality above, we have used implicitly the fact that for every $\varpi^{*} \in H_{\Gamma_{0}, \gamma}^{-1}(\Omega)$ and $\varpi \in D\left(\AA^{\frac{1}{2}}\right)$,

$$
\left\langle\varpi^{*}, \varpi\right\rangle_{{H_{0}, \gamma}_{-1}^{-1}(\Omega) \times H_{\Gamma_{0}, \gamma}^{1}(\Omega)}=\left\langle\varpi^{*}, \varpi\right\rangle\left[D\left(\AA^{\frac{1}{2}}\right)\right]^{\prime} \times D\left(\AA^{\frac{1}{2}}\right)
$$

- We denote the Hilbert space $\mathbf{H}_{\gamma}$ to be

$$
\mathbf{H}_{\gamma} \equiv D\left(\AA^{\frac{1}{2}}\right) \times H_{\Gamma_{0}, \gamma}^{1}(\Omega) \times L^{2}(\Omega)
$$

with the inner product

$$
\begin{aligned}
\left(\left[\begin{array}{l}
\omega_{1} \\
\omega_{2} \\
\theta
\end{array}\right],\left[\begin{array}{l}
\widehat{\omega}_{1} \\
\widehat{\omega}_{2} \\
\widehat{\theta}
\end{array}\right]\right)_{\mathbf{H}_{\gamma}}=\left(\AA^{\frac{1}{2}} \omega_{1}, \AA^{\frac{1}{2}} \widehat{\omega}_{1}\right)_{L^{2}(\Omega)} \\
+\left(P_{\gamma}^{\frac{1}{2}} \omega_{2}, P_{\gamma}^{\frac{1}{2}} \widehat{\omega}_{2}\right)_{L^{2}(\Omega)}+\beta(\theta, \widehat{\theta})_{L^{2}(\Omega)} .
\end{aligned}
$$

- With the above definitions, and making the denotation

$$
\text { (a) } \equiv A_{R}-\frac{\sigma}{\eta}-\AA G_{1} \gamma_{0}+\lambda \AA \AA G_{2} \gamma_{0},
$$

we then set $\mathcal{A}_{\gamma}: \mathbf{H}_{\gamma} \supset D\left(\mathcal{A}_{\gamma}\right) \rightarrow \mathbf{H}_{\gamma}$ to be

$$
\begin{array}{r}
\mathcal{A}_{\gamma} \equiv\left(\begin{array}{ccc}
\mathbf{I} & \mathbf{0} & \mathbf{0} \\
\mathbf{0} & P_{\gamma}^{-1} & \mathbf{0} \\
\mathbf{0} & \mathbf{0} & \mathbf{I}
\end{array}\right)\left(\begin{array}{ccc}
0 & \mathbf{I} & 0 \\
-\AA & 0 & \alpha(\mathbf{A}) \\
0 & -\frac{\alpha}{\beta} A_{D}\left(\mathbf{I}-D \gamma_{0}\right) & -\frac{\eta}{\beta} A_{R}
\end{array}\right) \\
\text { with } D\left(\mathcal{A}_{\gamma}\right)=\left\{\left[\omega_{0}, \omega_{1}, \theta_{0}\right] \in D\left(\AA^{\frac{1}{2}}\right) \times D\left(\AA^{\frac{1}{2}}\right) \times D\left(A_{R}\right)\right) \\
\text { such that } \left.\AA \omega_{0}+\alpha \AA G_{1} \gamma_{0} \theta_{0} \in H_{\Gamma_{0}, \gamma}^{-1}(\Omega)\right\}
\end{array}
$$


- We make the following denotations for the space of controllability:

$$
\begin{aligned}
U_{s} & =L^{2}\left(\Gamma_{1}\right) \times H^{-1}\left(\Gamma_{1}\right) \times H^{s}\left(\Gamma_{2}\right), \\
\mathcal{U}_{s} & =L^{2}\left(0, T ; L^{2}\left(\Gamma_{1}\right) \times H^{-1}\left(\Gamma_{1}\right)\right) \times H^{s}\left((0, T) \times \Gamma_{2}\right),
\end{aligned}
$$

where $s \geq 0$. We define the control operator $\mathcal{B}$ on $U_{s}$ by having for every $\bar{u}=\left[u_{1}, u_{2}, u_{3}\right] \in U_{s}$,

$$
\mathcal{B} \bar{u}=\left[\begin{array}{c}
0 \\
P_{\gamma}^{-1}\left[\AA G_{1} u_{1}+\AA G_{2} u_{2}\right] \\
\frac{\eta}{\beta} A_{R} R u_{3}
\end{array}\right] .
$$

Note that a priori the mapping $\mathcal{B}$ only makes sense as an element of $\mathcal{L}\left(U_{s}\right.$, $\left.\left[D\left(\mathcal{A}_{\gamma}^{*}\right)\right]^{\prime}\right)$, where $\mathbf{H}_{\gamma} \subset\left[D\left(\mathcal{A}_{\gamma}^{*}\right)\right]^{\prime}$. Indeed, for fixed $\bar{u}=\left[u_{1}, u_{2}, u_{3}\right] \in U_{s}$ one has, upon using the expression for the inverse $\mathcal{A}_{\gamma}^{-1}$ given in (4.2) below, and the definition of the elliptic operators $G_{1}, G_{2}$, and $R$ in (1.19) and (1.20) above, that

$$
\begin{aligned}
\mathcal{B} \bar{u} & =\mathcal{A}_{\gamma} \mathcal{A}_{\gamma}^{-1}\left[\begin{array}{c}
0 \\
P_{\gamma}^{-1}\left[\AA G_{1} u_{1}+\AA G_{2} u_{2}\right] \\
\frac{\eta}{\beta} A_{R} R u_{3}
\end{array}\right] \\
& =\mathcal{A}_{\gamma}\left[\begin{array}{c}
-G_{1} u_{1}-G_{2} u_{2}-\alpha \AA^{-1}(\boldsymbol{\beta}) R u_{3} \\
0 \\
-R u_{3}
\end{array}\right] \in\left[D\left(\mathcal{A}_{\gamma}^{*}\right)\right]^{\prime},
\end{aligned}
$$

where (\$) is as defined in (1.35).

- By duality, we have

$$
\begin{aligned}
U_{s}^{*} & =L^{2}\left(\Gamma_{1}\right) \times H^{1}\left(\Gamma_{1}\right) \times\left[H^{s}\left(\Gamma_{2}\right)\right]^{\prime}, \\
\mathcal{U}_{s}^{*} & =L^{2}\left(0, T ; L^{2}\left(\Gamma_{1}\right) \times H^{1}\left(\Gamma_{1}\right)\right) \times\left[H^{s}\left(0, T ; L^{2}\left(\Gamma_{2}\right)\right)\right]^{\prime},
\end{aligned}
$$

and $\mathcal{B}^{*} \in \mathcal{L}\left(D\left(\mathcal{A}_{\gamma}^{*}\right), U_{s}^{*}\right)$.

1.3.2. Abstract operator formulation. If we take the initial data $\left[\omega_{0}, \omega_{1}, \theta_{0}\right]$ to be in $\mathbf{H}_{\gamma}$, and control $\bar{u} \in \mathcal{U}_{s}$, where $\mathcal{U}_{s}$ is as defined in (1.37), then considering the operator definitions above, the coupled system (1.1) can be rewritten a fortiori as the operator theoretic model

$$
\frac{d}{d t}\left[\begin{array}{c}
\omega(t) \\
\omega_{t}(t) \\
\theta(t)
\end{array}\right]=\mathcal{A}_{\gamma}\left[\begin{array}{c}
\omega(t) \\
\omega_{t}(t) \\
\theta(t)
\end{array}\right]+\mathcal{B} \bar{u}(t), \quad\left[\begin{array}{c}
\omega(0) \\
\omega_{t}(0) \\
\theta(0)
\end{array}\right]=\left[\begin{array}{c}
\omega_{0} \\
\omega_{1} \\
\theta_{0}
\end{array}\right],
$$

with this equation having sense in $\left[D\left(\mathcal{A}_{\gamma}^{*}\right)\right]^{\prime}$ (a space strictly larger than $\mathbf{H}_{\gamma}$ ). Given the operator definitions for $\mathcal{A}_{\gamma}$ and $\mathcal{B}$ above, the solution $\left[\omega, \omega_{t}, \theta\right]$ to the ODE (1.41) (and so to the PDE (1.1)) is given by

$$
\left[\begin{array}{r}
\omega(\cdot) \\
\omega_{t}(\cdot) \\
\theta(\cdot)
\end{array}\right]=e^{\mathcal{A}_{\gamma}(\cdot)}\left[\begin{array}{c}
\omega_{0} \\
\omega_{1} \\
\theta_{0}
\end{array}\right]+\int_{0}^{(\cdot)} e^{\mathcal{A}_{\gamma}(\cdot-s)} \mathcal{B} \bar{u}(s) d s
$$


which by (1.39) and the convolution theorem is an element of $C\left([0, T] ;\left[D\left(\mathcal{A}_{\gamma}^{*}\right)\right]^{\prime}\right)$. With this representation of the solution $\left[\omega, \omega_{t}, \theta\right]$ in mind, we define the input $\rightarrow$ terminal state map $\mathcal{L}_{T} \in \mathcal{L}\left(\mathcal{U}_{s},\left[D\left(\mathcal{A}_{\gamma}^{*}\right)\right]^{\prime}\right)$ as

$$
\mathcal{L}_{T} \bar{u}=\int_{0}^{T} e^{\mathcal{A}_{\gamma}(T-s)} \mathcal{B} \bar{u}(s) d s .
$$

Taken as an unbounded operator from $\mathcal{U}_{s}$ into $\mathbf{H}_{\gamma}$, then $\mathcal{L}_{T}: D\left(\mathcal{L}_{T}\right) \subset \mathcal{U}_{s} \rightarrow \mathbf{H}_{\gamma}$ is closed and densely defined, with its domain of definition $D\left(\mathcal{L}_{T}\right)$ given to be

$$
D\left(\mathcal{L}_{T}\right)=\left\{\bar{u} \in \mathcal{U}_{s}: \mathcal{L}_{T} \bar{u} \in \mathbf{H}_{\gamma}\right\} .
$$

Its adjoint $\mathcal{L}_{T}^{*}: D\left(\mathcal{L}_{T}^{*}\right) \subset \mathbf{H}_{\gamma} \rightarrow \mathcal{U}_{s}^{*}$, where $\mathcal{U}_{s}^{*}$ is as given in (1.40), is likewise closed and densely defined, with

$$
D\left(\mathcal{L}_{T}^{*}\right)=\left\{\left[\phi_{0}, \phi_{1}, \psi_{0}\right] \in \mathbf{H}_{\gamma}: \mathcal{L}_{T}^{*}\left[\begin{array}{l}
\phi_{0} \\
\phi_{1} \\
\psi_{0}
\end{array}\right] \in \mathcal{U}_{s}^{*}\right\} .
$$

As we are concerned with obtaining exact controllability of the displacement $\left[\omega, \omega_{t}\right]$ only, we accordingly define the projection operator $\Pi: \mathbf{H}_{\gamma} \rightarrow D\left(\AA^{\frac{1}{2}}\right) \times$ $H_{\Gamma_{0}, \gamma}^{1}(\Omega)$ by

$$
\Pi\left[\begin{array}{c}
\varpi_{0} \\
\varpi_{1} \\
\vartheta_{0}
\end{array}\right]=\left[\begin{array}{l}
\varpi_{0} \\
\varpi_{1}
\end{array}\right] .
$$

Henceforth, the work here will be concerned with determining the surjectivity of the closed operator $\Pi \mathcal{L}_{T}, D\left(\Pi \mathcal{L}_{T}\right) \subset \mathcal{U}_{s} \rightarrow D\left(\AA^{\frac{1}{2}}\right) \times H_{\Gamma_{0}, \gamma}^{1}(\Omega)$, with

$$
\Pi \mathcal{L}_{T} \bar{u}=\Pi \int_{0}^{T} e^{\mathcal{A}_{\gamma}(T-s)} \mathcal{B} \bar{u}(s) d s,
$$

and with $D\left(\Pi \mathcal{L}_{T}\right)=D\left(\mathcal{L}_{T}\right)$. Determining the surjectivity of the operator $\Pi \mathcal{L}_{T}$ for some $T>0$ becomes our concern here, since it is equivalent to showing the exact controllability of the mechanical component $\left[\omega, \omega_{t}\right]$ to (1.1) (Theorem 1.2). This surjectivity for $\Pi \mathcal{L}_{T}$ is in turn equivalent to the existence of a certain observability inequality pertaining to the range of the adjoint $\mathcal{L}_{T}^{*} \Pi^{*}$ (the inequality (2.1) below), where $\mathcal{L}_{T}^{*} \Pi^{*}: D\left(\mathcal{L}_{T}^{*} \Pi^{*}\right) \subset D\left(\AA^{\frac{1}{2}}\right) \times H_{\Gamma_{0}, \gamma}^{1}(\Omega) \rightarrow \mathbf{H}_{\gamma}$ is likewise a closed densely defined operator (as $\mathcal{L}_{T}^{*}$ is), with its domain given by

$$
D\left(\mathcal{L}_{T}^{*} \Pi^{*}\right)=\left\{\left[\phi_{0}, \phi_{1}\right] \in D\left(\AA^{\frac{1}{2}}\right) \times H_{\Gamma_{0}}^{1}(\Omega):\left[\phi_{0}, \phi_{1}, 0\right] \in D\left(\mathcal{L}_{T}^{*}\right)\right\} .
$$

It is the injectivity condition (2.1) that we intend to directly verify. In order to rewrite this abstract inequality in "PDE form" (i.e., as the inequality (2.2) below), we need the following two propositions, the first of which is proved in the appendix below.

Proposition 1.5. The Hilbert space adjoint $\mathcal{A}_{\gamma}^{*}$ of $\mathcal{A}_{\gamma}$, as defined in (1.36), is given to be

$$
\begin{aligned}
& \mathcal{A}_{\gamma}^{*}=\left(\begin{array}{ccc}
\mathbf{I} & \mathbf{0} & \mathbf{0} \\
\mathbf{0} & P_{\gamma}^{-1} & \mathbf{0} \\
\mathbf{0} & \mathbf{0} & \mathbf{I}
\end{array}\right)\left(\begin{array}{ccc}
\mathbf{0} & -\mathbf{I} & \mathbf{0} \\
\AA & \mathbf{0} & -\alpha(\mathbf{o}) \\
\mathbf{0} & \frac{\alpha}{\beta} A_{D}\left(\mathbf{I}-D \gamma_{0}\right) & -\frac{\eta}{\beta} A_{R}
\end{array}\right), \\
& \text { with } D\left(\mathcal{A}_{\gamma}^{*}\right)=\left\{\left[\phi_{0}, \phi_{1}, \psi_{0}\right] \in D\left(\AA^{\frac{1}{2}}\right) \times D\left(\AA^{\frac{1}{2}}\right) \times D\left(A_{R}\right)\right. \\
& \text { such that } \left.\AA \phi_{0}+\alpha \AA G_{1} \gamma_{0} \psi_{0} \in H_{\Gamma_{0}, \gamma}^{-1}(\Omega)\right\}
\end{aligned}
$$


(above, (\$) is the same denotation made in (1.35)).

Remark 1.6. Using the semigroup $\left\{e^{\mathcal{A}_{\gamma}^{*} t}\right\}_{t \geq 0}$ generated by $\mathcal{A}_{\gamma}^{*}$, then for terminal data $\left[\phi_{0}, \phi_{1}, \psi_{0}\right] \in \mathbf{H}_{\gamma}$,

$$
\left[\begin{array}{c}
\phi(t) \\
\phi_{t}(t) \\
\psi(t)
\end{array}\right]=e^{\mathcal{A}_{\gamma}^{*}(T-t)}\left[\begin{array}{c}
\phi_{0} \\
\phi_{1} \\
\psi_{0}
\end{array}\right] \in C\left([0, T] ; \mathbf{H}_{\gamma}\right)
$$

is the solution to the following backward problem:

$$
\left\{\begin{array}{l}
\left\{\begin{array}{l}
\phi_{t t}-\gamma \Delta \phi_{t t}+\Delta^{2} \phi+\alpha \Delta \psi=0 \\
\beta \psi_{t}+\eta \Delta \psi-\sigma \psi-\alpha \Delta \phi_{t}=0
\end{array} \text { on }(0, \infty) \times \Omega,\right. \\
\phi=\frac{\partial \phi}{\partial \nu}=0 \text { on }(0, \infty) \times \Gamma_{0} \\
\left\{\begin{array}{l}
\Delta \phi+(1-\mu) B_{1} \phi+\alpha \psi=0 \\
\frac{\partial \Delta \phi}{\partial \nu}+(1-\mu) \frac{\partial B_{2} \phi}{\partial \tau}-\gamma \frac{\partial \phi_{t t}}{\partial \nu}+\alpha \frac{\partial \psi}{\partial \nu}=0
\end{array} \text { on }(0, \infty) \times \Gamma_{1},\right. \\
\frac{\partial \psi}{\partial \nu}+\lambda \psi=0 \text { on }(0, \infty) \times \Gamma, \lambda \geq 0 \\
{\left[\phi(T), \phi_{t}(T), \psi(T)\right]=\left[\phi_{0}, \phi_{1}, \psi_{0}\right] .}
\end{array}\right.
$$

Remark 1.7. For terminal data $\left[\phi_{0}, \phi_{1}, \psi_{0}\right]$ in $D\left(\mathcal{A}_{\gamma}^{*}\right)$, the two equations of $(1.51)$ may be written pointwise as

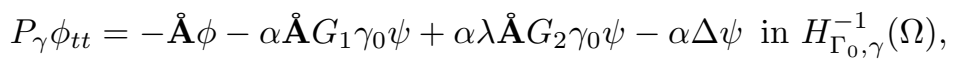

$$
\begin{aligned}
& \beta \psi_{t}=-\eta \Delta \psi+\sigma \psi+\alpha \Delta \phi_{t} \text { in } L^{2}(\Omega) \\
& {\left[\phi(T), \phi_{t}(T), \psi(T)\right]=\left[\phi_{0}, \phi_{1}, \psi_{0}\right] .}
\end{aligned}
$$

Remark 1.8. Since $\bar{\Gamma}_{0} \cap \bar{\Gamma}_{1}=\emptyset$, and $\Gamma$ is smooth, we can assume throughout that $D\left(\mathcal{A}_{\gamma}^{*}\right)$ is dense in the graph topology of $D\left(\mathcal{L}_{T}^{*}\right)$. be

Proposition 1.9. The adjoint $\mathcal{L}_{T}^{*}: D\left(\mathcal{L}_{T}^{*}\right) \subset \mathbf{H}_{\gamma} \rightarrow \mathcal{U}_{s}^{*}$ of $\mathcal{L}_{T}$ is computed to

$$
\mathcal{L}_{T}^{*}\left[\begin{array}{c}
\phi_{0} \\
\phi_{1} \\
\psi_{0}
\end{array}\right]=\left[\left.\frac{\partial \phi_{t}}{\partial \nu}\right|_{\Gamma_{1}},-\left.\phi_{t}\right|_{\Gamma_{1}},\left.\eta \psi\right|_{\Gamma_{2}}\right] \text { for all }\left[\begin{array}{c}
\phi_{0} \\
\phi_{1} \\
\psi_{0}
\end{array}\right] \in D\left(\mathcal{L}_{T}^{*}\right)
$$

where $\left[\left.\frac{\partial \phi_{t}}{\partial \nu}\right|_{\Gamma_{1}},\left.\phi_{t}\right|_{\Gamma_{1}},\left.\psi\right|_{\Gamma_{2}}\right]$ are boundary "traces" of the solution $\left[\phi, \phi_{t}, \psi\right]$ to the coupled system (1.51).

Proof. By Remark 1.8, it is enough to show the characterization in (1.55) for $\left[\phi_{0}, \phi_{1}, \psi_{0}\right] \in D\left(\mathcal{A}_{\gamma}^{*}\right)$. With this in mind, one has readily the classic representation

$$
\mathcal{L}_{T}^{*}\left[\begin{array}{c}
\phi_{0} \\
\phi_{1} \\
\psi_{0}
\end{array}\right]=\mathcal{B}^{*} e^{\mathcal{A}_{\gamma}^{*}(T-t)}\left[\begin{array}{c}
\phi_{0} \\
\phi_{1} \\
\psi_{0}
\end{array}\right] \text { for every }\left[\begin{array}{c}
\phi_{0} \\
\phi_{1} \\
\psi_{0}
\end{array}\right] \in D\left(\mathcal{A}_{\gamma}^{*}\right),
$$

where again, $\mathcal{B}^{*} \in \mathcal{L}\left(D\left(\mathcal{A}_{\gamma}^{*}\right), U_{s}^{*}\right)$ is the adjoint of $\mathcal{B}$. We must show that the righthand side of this equality may be written explicitly in "PDE form" as (1.55). To this end, for every $\left[u_{1}, u_{2}, u_{3}\right] \in \mathcal{U}_{s}$ and $\left[\phi_{0}, \phi_{1}, \psi_{0}\right] \in D\left(\mathcal{A}_{\gamma}^{*}\right)$, we have 


$$
\begin{aligned}
& \left\langle\mathcal{L}_{T}\left[\begin{array}{l}
u_{1} \\
u_{2} \\
u_{3}
\end{array}\right],\left[\begin{array}{l}
\phi_{0} \\
\phi_{1} \\
\psi_{0}
\end{array}\right]\right\rangle_{\left[D\left(\mathcal{A}_{\gamma}^{*}\right)\right]^{\prime} \times D\left(\mathcal{A}_{\gamma}^{*}\right)} \\
= & \left\langle\int_{0}^{T} e^{\mathcal{A}_{\gamma}(T-s)} \mathcal{B}\left[\begin{array}{l}
u_{1}(s) \\
u_{2}(s) \\
u_{3}(s)
\end{array}\right] d s,\left[\begin{array}{l}
\phi_{0} \\
\phi_{1} \\
\psi_{0}
\end{array}\right]\right\rangle_{\left[D\left(\mathcal{A}_{\gamma}^{*}\right)\right]^{\prime} \times D\left(\mathcal{A}_{\gamma}^{*}\right)} d s \\
= & \int_{0}^{T}\left\langle e^{\mathcal{A}_{\gamma}(T-s)} \mathcal{A}_{\gamma} \mathcal{A}_{\gamma}^{-1} \mathcal{B}\left[\begin{array}{l}
u_{1}(s) \\
u_{2}(s) \\
u_{3}(s)
\end{array}\right],\left[\begin{array}{l}
\phi_{0} \\
\phi_{1} \\
\psi_{0}
\end{array}\right]\right\rangle_{\left[D\left(\mathcal{A}_{\gamma}^{*}\right)\right]^{\prime} \times D\left(\mathcal{A}_{\gamma}^{*}\right)} d s \\
= & \int_{0}^{T}\left(\mathcal{A}_{\gamma}^{-1} \mathcal{B}\left[\begin{array}{l}
u_{1}(s) \\
u_{2}(s) \\
u_{3}(s)
\end{array}\right], e^{\mathcal{A}_{\gamma}^{*}(T-s)} \mathcal{A}_{\gamma}^{*}\left[\begin{array}{l}
\phi_{0} \\
\phi_{1} \\
\psi_{0}
\end{array}\right]\right)_{\mathbf{H}_{\gamma}} d s \\
= & \int_{0}^{T}\left(\left[\begin{array}{c}
-G_{1} u_{1}(s)-G_{2} u_{2}(s)-\alpha \AA^{-1}(\boldsymbol{\alpha}) R u_{3} \\
0
\end{array}\right], \mathcal{A}_{\gamma}^{*} e^{\mathcal{A}_{\gamma}^{*}(T-s)}\left[\begin{array}{c}
\phi_{0} \\
\phi_{1} \\
\psi_{0}
\end{array}\right]\right)_{\mathbf{H}_{\gamma}} d s .
\end{aligned}
$$

Noting that

$$
\left[\begin{array}{c}
\phi(t) \\
\phi_{t}(t) \\
\psi(t)
\end{array}\right] \equiv e^{\mathcal{A}_{\gamma}^{*}(T-t)}\left[\begin{array}{c}
\phi_{0} \\
\phi_{1} \\
\psi_{0}
\end{array}\right]
$$

gives the solution to the backward problem (1.51), we then use this relation, the definition of the adjoint $\mathcal{A}_{\gamma}^{*}$ in (1.49), and Proposition 4.1 of the appendix to obtain

$$
\begin{aligned}
& \left\langle\mathcal{L}_{T}\left[\begin{array}{l}
u_{1}(s) \\
u_{2}(s) \\
u_{3}(s)
\end{array}\right],\left[\begin{array}{l}
\phi_{0} \\
\phi_{1} \\
\psi_{0}
\end{array}\right]\right\rangle_{\left[D\left(\mathcal{A}_{\gamma}^{*}\right)\right]^{\prime} \times D\left(\mathcal{A}_{\gamma}^{*}\right)} \\
& =\int_{0}^{T}\left(\left[\begin{array}{c}
-G_{1} u_{1}(s)-G_{2} u_{2}(s)-\alpha \AA^{-1}(\boldsymbol{\beta}) R u_{3} \\
0 \\
-R u_{3}
\end{array}\right]\right. \text {, } \\
& \left.\left[\begin{array}{c}
-\phi_{t} \\
P_{\gamma}^{-1} \AA \mathbf{A} \phi-\alpha P_{\gamma}^{-1}(\boldsymbol{中}) \psi \\
\frac{\alpha}{\beta} A_{D}\left(\mathbf{I}-D \gamma_{0}\right) \phi_{t}-\frac{\eta}{\beta} A_{R} \psi
\end{array}\right]\right)_{\mathbf{H}_{\gamma}} d s \\
& =\int_{0}^{T}\left[\left(\AA^{\frac{1}{2}} G_{1} u_{1}, \AA^{\frac{1}{2}} \phi_{t}\right)_{L^{2}(\Omega)}+\left(\AA^{\frac{1}{2}} G_{2} u_{2}, \AA^{\frac{1}{2}} \phi_{t}\right)_{L^{2}(\Omega)}+\eta\left(R u_{3}, A_{R} \psi\right)_{L^{2}(\Omega)}\right] d t \\
& =\int_{0}^{T}\left[\left(u_{1}, G_{1}^{*} \AA \phi_{t}\right)_{L^{2}\left(\Gamma_{1}\right)}+\left\langle u_{2}, G_{2}^{*} \AA \phi_{t}\right\rangle_{H^{-1}\left(\Gamma_{1}\right) \times H^{1}\left(\Gamma_{1}\right)}\right] d t+\int_{0}^{T} \eta\left(u_{3}, \psi\right)_{L^{2}\left(\Gamma_{2}\right)} d t \\
& =\int_{0}^{T}\left[\left(u_{1}, \frac{\partial \phi_{t}}{\partial \nu}\right)_{L^{2}\left(\Gamma_{1}\right)}-\left\langle u_{2}, \phi_{t}\right\rangle_{H^{-1}\left(\Gamma_{1}\right) \times H^{1}\left(\Gamma_{1}\right)}\right] d t+\eta\left\langle u_{3}, \psi\right\rangle_{H^{s}\left((0, T) \Gamma_{2}\right) \times\left[H^{s}\left((0, T) \times \Gamma_{2}\right)\right]^{\prime}},
\end{aligned}
$$

thereby completing the proof of Proposition 1.9.

Immediately, we have Corollary 1.10. 
Corollary 1.10. The adjoint operator $\mathcal{L}_{T}^{*} \Pi^{*}: D\left(\mathcal{L}_{T}^{*} \Pi^{*}\right) \subset D\left(\AA^{\frac{1}{2}}\right) \times H_{\Gamma_{0}, \gamma}^{1}(\Omega) \rightarrow$ $\mathcal{U}_{s}^{*}$ is given by

$$
\mathcal{L}_{T}^{*} \Pi^{*}\left[\begin{array}{c}
\phi_{0} \\
\phi_{1}
\end{array}\right]=\left[\left.\frac{\partial \phi_{t}}{\partial \nu}\right|_{\Gamma_{1}},-\left.\phi_{t}\right|_{\Gamma_{1}},\left.\eta \psi\right|_{\Gamma_{2}}\right]
$$

for all $\left[\phi_{0}, \phi_{1}\right] \in D\left(\mathcal{L}_{T}^{*} \Pi^{*}\right)$, where $\left[\left.\frac{\partial \phi_{t}}{\partial \nu}\right|_{\Gamma_{1}},\left.\phi_{t}\right|_{\Gamma_{1}},\left.\psi\right|_{\Gamma_{2}}\right]$ are boundary traces of the solution $\left[\phi, \phi_{t}, \psi\right]$ to the following (backward) system:

$$
\left\{\begin{array}{l}
\left\{\begin{array}{l}
\phi_{t t}-\gamma \Delta \phi_{t t}+\Delta^{2} \phi+\alpha \Delta \psi=0 \\
\beta \psi_{t}+\eta \Delta \psi-\sigma \psi-\alpha \Delta \phi_{t}=0
\end{array} \quad \text { on }(0, \infty) \times \Omega,\right. \\
\phi=\frac{\partial \phi}{\partial \nu}=0 \text { on }(0, \infty) \times \Gamma_{0} \\
\left\{\begin{array}{l}
\Delta \phi+(1-\mu) B_{1} \phi+\alpha \psi=0 \\
\frac{\partial \Delta \phi}{\partial \nu}+(1-\mu) \frac{\partial B_{2} \phi}{\partial \tau}-\gamma \frac{\partial \phi_{t t}}{\partial \nu}+\alpha \frac{\partial \psi}{\partial \nu}=0
\end{array} \text { on }(0, \infty) \times \Gamma_{1},\right. \\
\frac{\partial \psi}{\partial \nu}+\lambda \psi=0 \text { on }(0, \infty) \times \Gamma, \lambda \geq 0 \\
{\left[\phi(T), \phi_{t}(T), \psi(T)\right]=\left[\phi_{0}, \phi_{1}, 0\right] .}
\end{array}\right.
$$

We conclude this section with a regularity result for the thermal component of the solution $\left[\phi, \phi_{t}, \psi\right]$ to (1.51), this being originally derived in [11] and [2] for the forward problem (1.1). Assuming terminal data $\left[\phi_{0}, \phi_{1}, \psi_{0}\right] \in D\left(\mathcal{A}_{\gamma}^{*}\right)$, we have, by using (1.50), the equality

$$
\frac{d}{d t}\left\|\left[\begin{array}{c}
\phi(t) \\
\phi_{t}(t) \\
\psi(t)
\end{array}\right]\right\|_{\mathbf{H}_{\gamma}}^{2}=-2\left(\mathcal{A}_{\gamma}^{*}\left[\begin{array}{c}
\phi(t) \\
\phi_{t}(t) \\
\psi(t)
\end{array}\right],\left[\begin{array}{c}
\phi(t) \\
\phi_{t}(t) \\
\psi(t)
\end{array}\right]\right)_{\mathbf{H}_{\gamma}} .
$$

Integrating this equation from 0 to $T$, performing computations similar to those performed for the proof of Proposition 1.9, recalling the characterization (1.15), and subsequently invoking a density argument, we have the following proposition.

Proposition 1.11. With terminal data $\left[\phi_{0}, \phi_{1}, \psi_{0}\right] \in \mathbf{H}_{\gamma}$, we have that the component $\psi$ of the solution of $(1.51)$ is an element of $L^{2}\left(0, \infty ; D\left(A_{R}^{\frac{1}{2}}\right)\right)$. Indeed, we have the following relation valid for all $T>0$ :

$$
\left\|\left[\begin{array}{l}
\phi_{0} \\
\phi_{1} \\
\psi_{0}
\end{array}\right]\right\|_{\mathbf{H}_{\gamma}}^{2}-\left\|\left[\begin{array}{c}
\phi(0) \\
\phi_{t}(0) \\
\psi(0)
\end{array}\right]\right\|_{\mathbf{H}_{\gamma}}^{2}=2 \eta \int_{0}^{T}\left\|A_{R}^{\frac{1}{2}} \psi\right\|_{L^{2}(\Omega)}^{2} d t .
$$

\section{Proof of Theorem 1.2.}

2.1. The necessary inequality. As stated above, showing the partial exact controllability of the system (1.1) for some time $T>0$ is equivalent to showing the surjectivity of the operator $\Pi \mathcal{L}_{T}: D\left(\mathcal{L}_{T}\right) \subset \mathcal{U}_{s} \rightarrow D\left(\AA^{\frac{1}{2}}\right) \times H_{\Gamma_{0}, \gamma}^{1}(\Omega)$, where $\Pi \mathcal{L}_{T}$ is as defined in (1.47) and with $D\left(\mathcal{L}_{T}\right)$ as defined in (1.44). Using the classical functional 
analysis (e.g., couple Lemma 3.8.18(i) and Theorem 6.5.10(ii) of [9]), the surjectivity of $\Pi \mathcal{L}_{T}$ for some time $T>0$ is tantamount to the existence of a constant $C_{T}>0$ such that following inequality is satisfied for all $\left[\phi_{0}, \phi_{1}\right] \in D\left(\mathcal{L}_{T}^{*} \Pi^{*}\right)$, where $D\left(\mathcal{L}_{T}^{*} \Pi^{*}\right)$ is as defined in (1.48):

$$
\left\|\mathcal{L}_{T}^{*}\left[\begin{array}{c}
\phi_{0} \\
\phi_{1} \\
0
\end{array}\right]\right\|_{\mathcal{U}_{s}^{*}} \geq C_{T}\left\|\left[\begin{array}{l}
\phi_{0} \\
\phi_{1}
\end{array}\right]\right\|_{D\left(\AA^{\frac{1}{2}}\right) \times H_{\Gamma_{0}, \gamma}^{1}(\Omega)} .
$$

Corollary 1.10 then gives that this abstract inequality above may be rewritten by having for all $\left[\phi_{0}, \phi_{1}\right] \in D\left(\mathcal{L}_{T}^{*} \Pi^{*}\right)$,

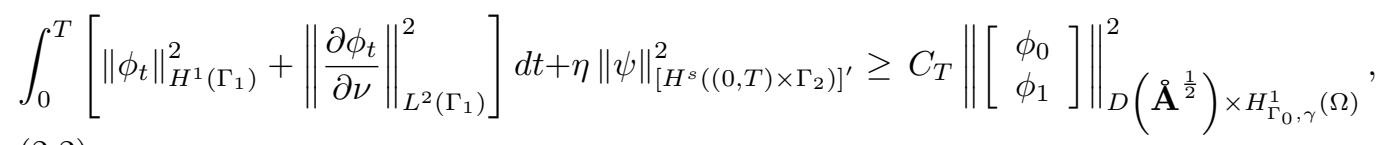

where $\left[\left.\frac{\partial \phi_{t}}{\partial \nu}\right|_{\Gamma_{1}},\left.\phi_{t}\right|_{\Gamma_{1}},\left.\psi\right|_{\Gamma_{2}}\right]$ are traces of the solution $\left[\phi, \phi_{t}, \psi\right]$ to the backward system (1.60) (this being "adjoint" with respect to (1.1)). So to prove the statement of partial exact controllability of the thermoelastic system (Theorem 1.2), it will hence suffice to establish the inequality (2.2) for $T>0$ large enough. With this end in mind, we make the following denotation for the mechanical "energy" of the system for $0 \leq t \leq T$ :

$$
E_{\phi}(t)=\frac{1}{2}\left[\left\|\AA^{\frac{1}{2}} \phi(t)\right\|_{L^{2}(\Omega)}^{2}+\left\|P_{\gamma}^{\frac{1}{2}} \phi_{t}(t)\right\|_{L^{2}(\Omega)}^{2}\right],
$$

where again $\left[\phi, \phi_{t}, \psi\right]$ solve the backward system (1.60). In addition, we will denote by I.o.t. $\left(\phi, \phi_{t}, \psi\right)$ ("lower order terms") any sum of terms that obey the following estimate for some constant $C_{T}$ :

$$
\begin{aligned}
& \text { I.o.t. }\left(\phi, \phi_{t}, \psi\right) \leq C_{T}\left(\|\phi\|_{L^{\infty}\left(0, T ; H^{\frac{3}{2}+\epsilon}(\Omega)\right.}^{2}+\left\|\phi_{t}\right\|_{L^{\infty}\left(0, T ; H^{\frac{1}{2}+\epsilon}(\Omega)\right.}^{2}\right) \\
& \left.\left.+\|\psi\|_{L^{2}\left(0, T ; H^{\frac{1}{2}+\epsilon}(\Omega)\right)}^{2}+\|\psi\|_{L^{\infty}\left(0, T ; H^{-\frac{1}{2}+\epsilon}(\Omega)\right.}^{2}\right)\right) .
\end{aligned}
$$

By way of establishing (2.2), the bulk of the work will entail the derivation of the following estimate.

TheOREM 2.1. For $T>0$ large enough, the solution $\left[\phi, \phi_{t}, \psi\right]$ to (1.51) with terminal data $\left[\phi_{0}, \phi_{1}, \psi_{0}\right] \in D\left(\mathcal{L}_{T}^{*}\right)$ satisfies the following inequality:

$$
\begin{array}{r}
\int_{0}^{T}\left[E_{\phi}(t)+\left\|A_{R}^{\frac{1}{2}} \psi\right\|_{L^{2}(\Omega)}^{2}\right] d t+E_{\phi}(0) \\
\leq C_{T}\left(\left\|\psi_{0}\right\|_{L^{2}(\Omega)}^{2}+\int_{0}^{T}\left\|\nabla \phi_{t}\right\|_{L^{2}\left(\Gamma_{1}\right)}^{2} d t+\text { I.o.t. }\left(\phi, \phi_{t}, \psi\right)\right) .
\end{array}
$$

This theorem will follow from a chain of results. Given the density of $D\left(\mathcal{A}_{\gamma}^{*}\right)$ in $D\left(\mathcal{L}_{T}^{*}\right)$ (see Remark 1.8) and the fact that the solution of (1.51) has the representation

$$
\left[\begin{array}{c}
\phi(t) \\
\phi_{t}(t) \\
\psi(t)
\end{array}\right]=e^{\mathcal{A}_{\gamma}^{*}(T-t)}\left[\begin{array}{c}
\phi_{0} \\
\phi_{1} \\
\psi_{0}
\end{array}\right]
$$


it will be enough to show inequality (2.5) for solutions $\left[\phi, \phi_{t}, \psi\right]$ to $(1.51)$ corresponding to terminal data in $D\left(\left[\mathcal{A}_{\gamma}^{*}\right]^{2}\right)$. Taking $\left[\phi_{0}, \phi_{1}, \psi_{0}\right] \in D\left(\left[\mathcal{A}_{\gamma}^{*}\right]^{2}\right)$, we then have that $\left[\phi, \phi_{t}, \psi\right]$ is an element of $C^{2}\left([0, T] ; \mathbf{H}_{\gamma}\right) \cap C^{1}\left([0, T] ; D\left(\mathcal{A}_{\gamma}^{*}\right)\right) \cap C\left([0, T] ; D\left(\left[\mathcal{A}_{\gamma}^{*}\right]^{2}\right)\right)$ and as such has the additional regularity (see [3, Theorem 2] and also [12]):

$$
\begin{aligned}
& \phi \in C\left([0, T] ; H^{4}(\Omega)\right) ; \phi_{t} \in C\left([0, T] ; H^{3}(\Omega)\right) ; \phi_{t t} \in C\left([0, T] ; D\left(\AA^{\frac{1}{2}}\right)\right), \\
& \psi_{t} \in C\left([0, T] ; D\left(A_{R}\right)\right), \\
& \phi-\gamma G_{2} \gamma_{1} \phi_{t t}+\alpha G_{1} \gamma_{0} \psi-\alpha \lambda G_{2} \gamma_{0} \psi \in C([0, T] ; D(\AA)) .
\end{aligned}
$$

This extra regularity of $\left[\phi, \phi_{t}, \psi\right]$, corresponding to smooth initial data, will justify the computations to be done below.

2.2. Proof of Theorem 2.1. As mentioned above, the terminal data $\left[\phi_{0}, \phi_{1}, \psi_{0}\right]$ will be considered to be in $D\left(\left[\mathcal{A}_{\gamma}^{*}\right]^{2}\right)$; accordingly the corresponding solution $\left[\phi, \phi_{t}, \psi\right]$ of (1.51) will be a classical one, with the regularity posted in (2.7). With the end in mind of deriving the estimate (2.2), we start by making the substitution

$$
\widehat{\phi}(t)=e^{-\xi t} \phi(t) \text { and } \widehat{\psi}(t)=e^{-\xi t} \psi(t),
$$

where parameter $\xi \in \mathbb{R}$ is to be determined. Necessarily then $\left[\widehat{\phi}, \widehat{\phi}_{t}, \widehat{\psi}\right]$ solves the coupled (backward) system

$$
\left\{\begin{array}{l}
\left\{\begin{array}{l}
\left(\xi^{2} \widehat{\phi}+2 \xi \widehat{\phi}_{t}+\widehat{\phi}_{t t}\right)-\gamma \Delta\left(\xi^{2} \widehat{\phi}+2 \xi \widehat{\phi}_{t}+\widehat{\phi}_{t t}\right)+\Delta^{2} \widehat{\phi}+\alpha \Delta \widehat{\psi}=0 \\
\beta\left(\xi \widehat{\psi}+\widehat{\psi}_{t}\right)+\eta \Delta \widehat{\psi}-\sigma \widehat{\psi}-\alpha \Delta\left(\xi \widehat{\phi}+\widehat{\phi}_{t}\right)=0
\end{array} \quad \text { on }(0, \infty) \times \Omega,\right. \\
\widehat{\phi}=\frac{\partial \widehat{\phi}}{\partial \nu}=0 \text { on }(0, \infty) \times \Gamma_{0}, \\
\left\{\begin{array}{l}
\Delta \widehat{\phi}+(1-\mu) B_{1} \widehat{\phi}+\alpha \widehat{\psi}=0 \\
\frac{\partial \Delta \widehat{\phi}}{\partial \nu}+(1-\mu) \frac{\partial B_{2} \widehat{\phi}}{\partial \tau}-\gamma \frac{\partial}{\partial \nu}\left(\xi^{2} \widehat{\phi}+2 \xi \widehat{\phi}_{t}+\widehat{\phi}_{t t}\right)+\alpha \frac{\partial \widehat{\psi}}{\partial \nu}=0
\end{array} \quad \text { on }(0, \infty) \times \Gamma_{1},\right. \\
\frac{\partial \widehat{\psi}}{\partial \nu}+\lambda \widehat{\psi}=0 \text { on }(0, \infty) \times \Gamma, \lambda \geq 0, \\
{\left[\widehat{\phi}(T), \widehat{\phi}_{t}(T), \widehat{\psi}(T)\right]=\left[e^{-\xi T} \phi_{0},-\xi e^{-\xi T} \phi_{0}+e^{-\xi T} \phi_{1}, e^{-\xi T} \psi_{0}\right] .}
\end{array}\right.
$$

Since $\left[\phi_{0}, \phi_{1}, \psi_{0}\right] \in D\left(\left[\mathcal{A}_{\gamma}^{*}\right]^{2}\right)$, the extra regularity in $(2.7)$ gives that $[\widehat{\phi}, \widehat{\phi} t, \widehat{\psi}]$ is a classical (not just weak) solution of (2.9); accordingly, we can rewrite (2.9) abstractly as (see Remark 1.7 and (1.31))

$$
\begin{aligned}
& \left(\xi^{2} \widehat{\phi}+2 \xi \widehat{\phi}_{t}+\widehat{\phi}_{t t}\right)-\gamma \Delta\left(\xi^{2} \widehat{\phi}+2 \xi \widehat{\phi}_{t}+\widehat{\phi}_{t t}\right)-\gamma \AA G_{2} \gamma_{1}\left(\xi^{2} \widehat{\phi}+2 \xi \widehat{\phi}_{t}+\widehat{\phi}_{t t}\right) \\
& +\AA \widehat{\AA} \widehat{\phi}+\alpha \AA G_{1} \gamma_{0} \widehat{\psi}-\alpha \lambda \AA G_{2} \gamma_{0} \widehat{\psi}+\alpha \Delta \widehat{\psi}=0 \text { in } H_{\Gamma_{0}, \gamma}^{-1}(\Omega), \\
& \text { (2.11) } \beta\left(\xi \widehat{\psi}+\widehat{\psi}_{t}\right)+\eta \Delta \widehat{\psi}-\sigma \widehat{\psi}-\alpha \Delta\left(\xi \widehat{\phi}+\widehat{\phi}_{t}\right)=0 \text { in } L^{2}(\Omega) \text {, } \\
& {\left[\widehat{\phi}(T), \widehat{\phi}_{t}(T), \widehat{\psi}(T)\right]=\left[e^{-\xi T} \phi_{0},-\xi e^{-\xi T} \phi_{0}+e^{-\xi T} \phi_{1}, e^{-\xi T} \psi_{0}\right] .}
\end{aligned}
$$


Now multiplying the heat equation (2.11) by $\frac{\alpha}{\eta}$ and adding it to the Kirchoff plate (2.10), and subsequently taking the parameter $\xi$ to be $\xi \equiv \frac{\alpha^{2}}{2 \gamma \eta}$, we obtain the single equation

$$
\begin{gathered}
\widehat{\phi}_{t t}-\gamma \Delta \widehat{\phi}_{t t}+\AA \widehat{\mathbf{A}}-\gamma \AA G_{2} \gamma_{1}\left(\xi^{2} \widehat{\phi}+2 \xi \widehat{\phi}_{t}+\widehat{\phi}_{t t}\right)+\alpha \AA G_{1} \gamma_{0} \widehat{\psi}-\alpha \lambda \AA G_{2} \gamma_{0} \widehat{\psi} \\
=c_{0} \widehat{\psi}+c_{1} \widehat{\psi}+c_{2} \widehat{\phi}+c_{3} \widehat{\phi}_{t}+c_{4} \Delta \widehat{\phi}, \\
{\left[\widehat{\phi}(T), \widehat{\phi}_{t}(T), \widehat{\psi}(T)\right]=\left[e^{-\xi T} \phi_{0},-\xi e^{-\xi T} \phi_{0}+e^{-\xi T} \phi_{1}, e^{-\xi T} \psi_{0}\right],}
\end{gathered}
$$

where the constants $c_{0}=\frac{\alpha^{3} \beta}{2 \gamma \eta^{2}}-\frac{\alpha \sigma}{\eta}, c_{1}=\frac{\alpha \beta}{\eta}, c_{2}=-\frac{\alpha^{4}}{4 \gamma^{2} \eta^{2}}, c_{3}=-\frac{\alpha^{2}}{\gamma \eta}$, and $c_{4}=$ $-\frac{\alpha^{4}}{4 \gamma \eta^{2}}$. (Note that the particular choice of $\xi$ made here eliminates the higher order term $\Delta \widehat{\phi}_{t}$.) System (2.13)-(2.14) may be rewritten in PDE form as the Kirchoff plate equation

$$
\left\{\begin{array}{l}
\widehat{\phi}_{t t}-\gamma \Delta \widehat{\phi}_{t t}+\Delta^{2} \widehat{\phi}=c_{0} \widehat{\psi}+c_{1} \widehat{\psi}_{t}+c_{2} \widehat{\phi}+c_{3} \widehat{\phi}_{t}+c_{4} \Delta \widehat{\phi} \quad \text { on }(0, \infty) \times \Omega, \\
\widehat{\phi}=\frac{\partial \widehat{\phi}}{\partial \nu}=0 \text { on }(0, \infty) \times \Gamma_{0}, \\
\left\{\begin{array}{l}
\Delta \widehat{\phi}+(1-\mu) B_{1} \widehat{\phi}=-\alpha \widehat{\psi} \\
\frac{\partial \Delta \widehat{\phi}}{\partial \nu}+(1-\mu) \frac{\partial B_{2} \widehat{\phi}}{\partial \tau}-\gamma \frac{\partial \widehat{\phi}_{t t}}{\partial \nu}=\gamma \frac{\partial}{\partial \nu}\left(\xi^{2} \widehat{\phi}+2 \xi \widehat{\phi}_{t}\right)-\alpha \frac{\partial \widehat{\psi}}{\partial \nu} \quad \text { on }(0, \infty) \times \Gamma_{1}, \\
{\left[\widehat{\phi}(T), \widehat{\phi}_{t}(T), \widehat{\psi}(T)\right]=\left[e^{-\xi T} \phi_{0},-\xi e^{-\xi T} \phi_{0}+e^{-\xi T} \phi_{1}, e^{-\xi T} \psi_{0}\right] .}
\end{array}\right.
\end{array}\right.
$$

As $\widehat{\phi}-\gamma G_{2} \gamma_{1}\left(\xi^{2} \widehat{\phi}+2 \xi \widehat{\phi}_{t}+\widehat{\phi}_{t t}\right)+\alpha G_{1} \gamma_{0} \widehat{\psi}-\alpha \lambda G_{2} \gamma_{0} \widehat{\psi} \in C([0, T] ; D(\AA))$ (using the last containment in $(2.7))$, then $\left[\widehat{\phi}, \widehat{\phi}_{t}\right]$ is a classical solution of $(2.15)$.

We note at this point that one can readily derive the trace estimate Lemma 4.5 (of the appendix below) for the plate component $\left.\Delta \widehat{\phi}\right|_{\Gamma_{0}}$ of the solution $\left[\widehat{\phi}, \widehat{\phi}_{t}, \widehat{\psi}\right]$ of (2.9). The proof of this is relegated to the appendix, since it is entirely analogous to that shown for the forward problem in [3] and [4]. This estimate will be critical in the proof of the following lemma, which gives an energy relation for the mechanical variable.

Lemma 2.2. (a) The solution $\left[\widehat{\phi}, \widehat{\phi}_{t}, \widehat{\psi}\right]$ to (2.9) satisfies the following relation for all $s$ and $\tau \in[0, T]$ :

$$
\left.E_{\widehat{\phi}}(t)\right|_{t=s} ^{t=\tau}=\mathcal{F}(s, \tau),
$$

where $E_{\widehat{\phi}}(\tau)$ is the mechanical energy function defined in $(2.3)$ and $\mathcal{F}(\cdot, \cdot)$ is a function (defined below in (2.34)) that obeys the following estimate for all $s$ and $\tau \in[0, T]$ and $\epsilon>0$ :

$$
\begin{aligned}
& \mathcal{F}(s, \tau) \leq C_{\epsilon} \int_{0}^{T}\left\|\nabla \widehat{\phi}_{t}\right\|_{L^{2}\left(\Gamma_{1}\right)}^{2} d t+\epsilon \int_{0}^{T}\left[E_{\widehat{\phi}}(t)+\left\|A_{R}^{\frac{1}{2}} \widehat{\psi}(t)\right\|_{L^{2}(\Omega)}^{2}\right] d t \\
& +\epsilon\left(E_{\widehat{\phi}}(s)+E_{\widehat{\phi}}(\tau)\right)+\text { l.o.t. }\left(\widehat{\phi}, \widehat{\phi}_{t} \widehat{\psi}\right) .
\end{aligned}
$$

(b) For $\epsilon>0$ small enough, the solution $\left[\widehat{\phi}, \widehat{\phi}_{t}, \widehat{\psi}\right]$ to (2.9) satisfies the following 
estimate for all $s$ and $\tau \in[0, T]$ :

$$
\begin{aligned}
& E_{\widehat{\phi}}(\tau) \leq\left(\frac{1+\epsilon}{1-\epsilon}\right) E_{\widehat{\phi}}(s)+C_{\epsilon} \int_{0}^{T}\left\|\nabla \widehat{\phi}_{t}\right\|_{L^{2}\left(\Gamma_{1}\right)}^{2} d t \\
& +\frac{\epsilon}{1-\epsilon} \int_{0}^{T}\left[E_{\widehat{\phi}}(t)+\left\|A_{R}^{\frac{1}{2}} \widehat{\psi}(t)\right\|_{L^{2}(\Omega)}^{2}\right] d t+\text { I.o.t. }(\widehat{\phi}, \widehat{\phi} t \widehat{\psi}) .
\end{aligned}
$$

Above, the constant $C_{\epsilon}$ is independent of time.

Proof. We take the duality pairing of the abstract equation (2.13) with $\widehat{\phi}_{t}$ and integrate in time and space so as to get

$$
\begin{aligned}
& \int_{s}^{\tau}\left[\left\langle\widehat{\phi}_{t t}-\gamma \Delta \widehat{\phi}_{t t}-\gamma \AA G_{2} \gamma_{1} \widehat{\phi}_{t t}, \widehat{\phi}_{t}\right\rangle_{H_{\Gamma_{0}}^{-1}(\Omega) \times H_{\Gamma_{0}}^{1}(\Omega)}+\left\langle\AA \widehat{\mathbf{\phi}}, \widehat{\phi}_{t}\right\rangle\left[D\left(\AA^{\frac{1}{2}}\right)\right]^{\prime} \times D\left(\AA^{\frac{1}{2}}\right)\right] d t \\
& =\int_{s}^{\tau}\left\langle\gamma \AA G_{2} \gamma_{1}\left(\xi^{2} \widehat{\phi}+2 \xi \widehat{\phi}_{t}\right)-\alpha \AA G_{1} \gamma_{0} \widehat{\psi}+\alpha \lambda \AA G_{2} \gamma_{0} \widehat{\psi}, \widehat{\phi}_{t}\right\rangle\left[D\left(\AA^{\frac{1}{2}}\right)\right]^{\prime} \times D\left(\AA^{\frac{1}{2}}\right) d t \\
& +\int_{s}^{\tau}\left(c_{0} \widehat{\psi}+c_{1} \widehat{\psi}_{t}+c_{2} \widehat{\phi}+c_{3} \widehat{\phi}_{t}+c_{4} \Delta \widehat{\phi}, \widehat{\phi}_{t}\right)_{L^{2}(\Omega)} d t .
\end{aligned}
$$

(Note that here we are using implicitly the fact that the terminal data $\left[\phi_{0}, \phi_{1}, \psi_{0}\right]$ being in $D\left(\mathcal{A}_{\gamma}^{*}\right)$ implies that $\AA \widehat{\phi}+\gamma \AA G_{2} \gamma_{1}\left(\xi^{2} \widehat{\phi}+2 \xi \widehat{\phi}_{t}+\widehat{\phi}_{t t}\right)-\alpha \AA G_{1} \gamma_{0} \widehat{\psi}+\alpha \lambda \AA G_{2} \gamma_{0} \widehat{\psi}$ is an element of $C\left([0, T] ; H_{\Gamma_{0}}^{-1}(\Omega)\right)$.) Second, denoting $A_{D}^{-1}$ to be the inverse of the elliptic operator defined in (1.12), we multiply the $\operatorname{PDE}(2.15)$ by $-\frac{c_{1}}{\gamma} A_{D}^{-1} \widehat{\psi}$, and subsequently integrate in time and space so as to get

$-\frac{c_{1}}{\gamma} \int_{s}^{\tau}\left(\widehat{\phi}_{t t}-\gamma \Delta \widehat{\phi}_{t t}+\Delta^{2} \widehat{\phi}-\left[c_{0} \widehat{\psi}+c_{1} \widehat{\psi}_{t}+c_{2} \widehat{\phi}+c_{3} \widehat{\phi}_{t}+c_{4} \Delta \widehat{\phi}\right], A_{D}^{-1} \widehat{\psi}\right)_{L^{2}(\Omega)} d t=0$.

(A1) Rewriting (2.19). Using equality (1.31) and the characterizations in (1.23), we have upon the taking of adjoints that (2.19) may be rewritten as

$$
\begin{aligned}
& \left.E_{\widehat{\phi}}(t)\right|_{t=s} ^{t=\tau}=\int_{s}^{\tau}\left(c_{0} \widehat{\psi}+c_{2} \widehat{\phi}+c_{3} \widehat{\phi}_{t}+c_{4} \Delta \widehat{\phi}, \widehat{\phi}_{t}\right)_{L^{2}(\Omega)} d t+\int_{s}^{\tau} c_{1}\left(\widehat{\psi}_{t}, \widehat{\phi}_{t}\right)_{L^{2}(\Omega)} d t \\
& -\int_{s}^{\tau}\left[\left(\gamma \xi^{2} \frac{\partial \widehat{\phi}}{\partial \nu}+2 \gamma \xi \frac{\partial \widehat{\phi}_{t}}{\partial \nu}+\alpha \lambda \gamma_{0} \widehat{\psi}, \widehat{\phi}_{t}\right)_{L^{2}\left(\Gamma_{1}\right)}+\alpha\left(\widehat{\psi}, \frac{\partial \widehat{\phi}_{t}}{\partial \nu}\right)_{L^{2}\left(\Gamma_{1}\right)}\right] d t .
\end{aligned}
$$

(A2) Rewriting (2.20). (i) An integration by parts, the use of the heat equation (2.11), and the fact that $A_{R} \widehat{\psi}=-\Delta \widehat{\psi}+\Delta D \gamma_{0} \widehat{\psi}+\frac{\sigma}{\eta} \widehat{\psi}=A_{D}\left(\mathbf{I}-D \gamma_{0}\right) \widehat{\psi}+\frac{\sigma}{\eta} \widehat{\psi}$ yield

$$
\begin{aligned}
& \int_{s}^{\tau}-\frac{c_{1}}{\gamma}\left(\widehat{\phi}_{t t}, A_{D}^{-1} \widehat{\psi}\right)_{L^{2}(\Omega)} d t=\left[-\frac{c_{1}}{\gamma}\left(\widehat{\phi}_{t}, A_{D}^{-1} \widehat{\psi}\right)_{L^{2}(\Omega)}\right]_{s}^{\tau} \\
& +\frac{c_{1}}{\gamma} \int_{s}^{\tau}\left(\widehat{\phi}_{t}, A_{D}^{-1} \widehat{\psi}_{t}\right)_{L^{2}(\Omega)} d t
\end{aligned}
$$




$$
\begin{aligned}
= & {\left[-\frac{c_{1}}{\gamma}\left(\widehat{\phi}_{t}, A_{D}^{-1} \widehat{\psi}\right)_{L^{2}(\Omega)}\right]_{s}^{\tau} } \\
& +\frac{c_{1}}{\gamma} \int_{s}^{\tau}\left(\widehat{\phi}_{t},\left(\frac{\sigma}{\beta}-\xi\right) A_{D}^{-1} \widehat{\psi}+\frac{\eta}{\beta}\left(\mathbf{I}-D \gamma_{0}\right) \widehat{\psi}\right)_{L^{2}(\Omega)} d t \\
& -\frac{c_{1}}{\gamma} \int_{s}^{\tau}\left(\widehat{\phi}_{t}, \frac{\alpha \xi}{\beta}\left(\mathbf{I}-D \gamma_{0}\right) \widehat{\phi}+\frac{\alpha}{\beta}\left(\mathbf{I}-D \gamma_{0}\right) \widehat{\phi}_{t}\right)_{L^{2}(\Omega)} d t
\end{aligned}
$$

(ii) An integration by parts and employment of Green's theorem yield

$$
\begin{aligned}
& \int_{s}^{\tau} c_{1}\left(\Delta \widehat{\phi}_{t t}, A_{D}^{-1} \widehat{\psi}\right)_{L^{2}(\Omega)} d t=-\int_{s}^{\tau} c_{1}\left(\nabla \widehat{\phi}_{t t}, \nabla A_{D}^{-1} \widehat{\psi}\right)_{L^{2}(\Omega)} d t \\
= & -c_{1}\left[\left(\nabla \widehat{\phi}_{t}, \nabla A_{D}^{-1} \widehat{\psi}\right)_{L^{2}(\Omega)}\right]_{s}^{\tau}+c_{1} \int_{s}^{\tau}\left(\nabla \widehat{\phi}_{t}, \nabla A_{D}^{-1} \widehat{\psi}_{t}\right)_{L^{2}(\Omega)} d t \\
= & -c_{1}\left[\left(\nabla \widehat{\phi}_{t}, \nabla A_{D}^{-1} \widehat{\psi}\right)_{L^{2}(\Omega)}\right]_{s}^{\tau}+c_{1} \int_{s}^{\tau}\left(\widehat{\phi}_{t}, A_{D} A_{D}^{-1} \widehat{\psi}_{t}\right)_{L^{2}(\Omega)} d t \\
& +c_{1} \int_{s}^{\tau}\left(\widehat{\phi}_{t}, \frac{\partial A_{D}^{-1} \widehat{\psi}_{t}}{\partial \nu}\right)_{L^{2}\left(\Gamma_{1}\right)} d t \\
= & -c_{1}\left[\left(\nabla \widehat{\phi}_{t}, \nabla A_{D}^{-1} \widehat{\psi}\right)_{L^{2}(\Omega)}\right]_{s}^{\tau}+c_{1} \int_{s}^{\tau}\left(\widehat{\phi}_{t}, \widehat{\psi}_{t}\right)_{L^{2}(\Omega)} d t \\
& +c_{1} \int_{s}^{\tau}\left(\widehat{\phi}_{t},\left(\frac{\sigma}{\beta}-\xi\right) \frac{\partial A_{D}^{-1} \widehat{\psi}}{\partial \nu}+\frac{\eta}{\beta} \frac{\partial\left(\mathbf{I}-D \gamma_{0}\right) \widehat{\psi}}{\partial \nu}\right)_{L^{2}\left(\Gamma_{1}\right)} d t \\
& -c_{1} \int_{s}^{\tau}\left(\widehat{\phi}_{t}, \frac{\alpha \xi}{\beta} \frac{\partial\left(\mathbf{I}-D \gamma_{0}\right) \widehat{\phi}}{\partial \nu}+\frac{\alpha}{\beta} \frac{\partial\left(\mathbf{I}-D \gamma_{0}\right) \widehat{\phi}_{t}}{\partial \nu}\right)_{L^{2}\left(\Gamma_{1}\right)} d t .
\end{aligned}
$$

(iii) Through the use of Green's theorem (1.9) and the boundary conditions in (2.15), we obtain

$$
\begin{aligned}
& -\int_{s}^{\tau} \frac{c_{1}}{\gamma}\left(\Delta^{2} \widehat{\phi}, A_{D}^{-1} \widehat{\psi}\right)_{L^{2}(\Omega)} d t \\
= & -\frac{c_{1}}{\gamma} \int_{s}^{\tau} a\left(\widehat{\phi}, A_{D}^{-1} \widehat{\psi}\right) d t-\frac{\alpha c_{1}}{\gamma} \int_{s}^{\tau}\left(\widehat{\psi}, \frac{\partial A_{D}^{-1} \widehat{\psi}}{\partial \nu}\right)_{L^{2}\left(\Gamma_{1}\right)} d t \\
& +\frac{c_{1}}{\gamma} \int_{s}^{\tau}\left(\Delta \widehat{\phi}, \frac{\partial A_{D}^{-1} \widehat{\psi}}{\partial \nu}\right)_{L^{2}\left(\Gamma_{0}\right)} d t
\end{aligned}
$$

(where we have used the fact that $\left.\widehat{\phi}\right|_{\Gamma_{0}}=\left.\frac{\partial \widehat{\phi}}{\partial \nu}\right|_{\Gamma_{0}}=0$ implies $\left.B_{1} \widehat{\phi}\right|_{\Gamma_{0}}=0$; see [11]).

Jointly then, equalities (2.20) and (2.22)-(2.24) give the relation 


$$
\begin{aligned}
& 0=-c_{1} \int_{s}^{\tau}\left(\widehat{\phi}_{t}, \widehat{\psi}_{t}\right)_{L^{2}(\Omega)} d t-\frac{c_{1}}{\gamma} \int_{s}^{\tau}\left(\widehat{\phi}_{t},\left(\frac{\sigma}{\beta}-\xi\right) A_{D}^{-1} \widehat{\psi}+\frac{\eta}{\beta}\left(\mathbf{I}-D \gamma_{0}\right) \widehat{\psi}\right)_{L^{2}(\Omega)} d t \\
& +\frac{c_{1}}{\gamma} \int_{s}^{\tau}\left(\widehat{\phi}_{t}, \frac{\alpha \xi}{\beta}\left(\mathbf{I}-D \gamma_{0}\right) \widehat{\phi}+\frac{\alpha}{\beta}\left(\mathbf{I}-D \gamma_{0}\right) \widehat{\phi}_{t}\right)_{L^{2}(\Omega)} d t \\
& -c_{1} \int_{s}^{\tau}\left(\widehat{\phi}_{t},\left(\frac{\sigma}{\beta}-\xi\right) \frac{\partial A_{D}^{-1} \widehat{\psi}}{\partial \nu}+\frac{\eta}{\beta} \frac{\partial\left(\mathbf{I}-D \gamma_{0}\right) \widehat{\psi}}{\partial \nu}\right)_{L^{2}\left(\Gamma_{1}\right)} d t \\
& +c_{1} \int_{s}^{\tau}\left(\widehat{\phi}_{t}, \frac{\alpha \xi}{\beta} \frac{\partial\left(\mathbf{I}-D \gamma_{0}\right) \widehat{\phi}}{\partial \nu}+\frac{\alpha}{\beta} \frac{\partial\left(\mathbf{I}-D \gamma_{0}\right) \widehat{\phi}_{t}}{\partial \nu}\right)_{L^{2}\left(\Gamma_{1}\right)} d t \\
& +\frac{c_{1}}{\gamma} \int_{s}^{\tau} a\left(\widehat{\phi}, A_{D}^{-1} \widehat{\psi}\right) d t+\frac{\alpha c_{1}}{\gamma} \int_{s}^{\tau}\left(\widehat{\psi}, \frac{\partial A_{D}^{-1} \widehat{\psi}}{\partial \nu}\right)_{L^{2}\left(\Gamma_{1}\right)} d t \\
& -\frac{c_{1}}{\gamma} \int_{s}^{\tau}\left(\Delta \widehat{\phi}, \frac{\partial A_{D}^{-1} \widehat{\psi}}{\partial \nu}\right)_{L^{2}\left(\Gamma_{0}\right)} d t \\
& -\int_{s}^{\tau}\left(c_{0} \widehat{\psi}+c_{1} \widehat{\psi}+c_{2} \widehat{\phi}+c_{3} \widehat{\phi}_{t}+c_{4} \Delta \widehat{\phi}, \frac{c_{1}}{\gamma} A_{D}^{-1} \widehat{\psi}\right)_{L^{2}(\Omega)} d t \\
& +c_{1}\left[\left(\nabla \widehat{\phi}_{t}, \nabla A_{D}^{-1} \widehat{\psi}\right)_{L^{2}(\Omega)}+\frac{1}{\gamma}\left(\widehat{\phi}_{t}, A_{D}^{-1} \widehat{\psi}\right)_{L^{2}(\Omega)}\right]_{s} \cdot
\end{aligned}
$$

Summing the relations (2.21) and (2.25), we obtain

$$
\begin{aligned}
& \left.E_{\widehat{\phi}}(t)\right|_{t=s} ^{t=\tau}=\int_{s}^{\tau}\left(c_{0} \widehat{\psi}+c_{2} \widehat{\phi}+c_{3} \widehat{\phi}_{t}+c_{4} \Delta \widehat{\phi}, \widehat{\phi}_{t}-\frac{c_{1}}{\gamma} A_{D}^{-1} \widehat{\psi}\right)_{L^{2}(\Omega)} d t \\
& -\int_{s}^{\tau}\left[\left(\gamma \xi^{2} \frac{\partial \widehat{\phi}}{\partial \nu}+2 \gamma \xi \frac{\partial \widehat{\phi}_{t}}{\partial \nu}+\alpha \lambda \gamma_{0} \widehat{\psi}, \widehat{\phi}_{t}\right)_{L^{2}\left(\Gamma_{1}\right)}+\alpha\left(\widehat{\psi}, \frac{\partial \widehat{\phi}_{t}}{\partial \nu}\right)_{L^{2}\left(\Gamma_{1}\right)}\right] d t \\
& -\frac{c_{1}}{\gamma} \int_{s}^{\tau}\left(\widehat{\phi}_{t},\left(\frac{\sigma}{\beta}-\xi\right) A_{D}^{-1} \widehat{\psi}+\frac{\eta}{\beta}\left(\mathbf{I}-D \gamma_{0}\right) \widehat{\psi}\right)_{L^{2}(\Omega)} d t+c_{1}\left[\left(\nabla \widehat{\phi}_{t}, \nabla A_{D}^{-1} \widehat{\psi}\right)_{L^{2}(\Omega)}\right]_{s}^{\tau} \\
& +c_{1} \int_{s}^{\tau}\left[\left(\frac{\widehat{\phi}_{t}}{\gamma}, \frac{\alpha \xi}{\beta}\left(\mathbf{I}-D \gamma_{0}\right) \widehat{\phi}+\frac{\alpha}{\beta}\left(\mathbf{I}-D \gamma_{0}\right) \widehat{\phi}_{t}\right)_{L^{2}(\Omega)}\right. \\
& \left.-\left(\widehat{\phi},\left(\frac{\sigma}{\beta}-\xi\right) \frac{\partial A_{D}^{-1} \widehat{\psi}}{\partial \nu}+\frac{\eta}{\beta} \frac{\partial\left(\mathbf{I}-D \gamma_{0}\right) \widehat{\psi}}{\partial \nu}\right)_{L^{2}\left(\Gamma_{1}\right)}\right] d t \\
& +c_{1} \int_{s}^{\tau}\left(\widehat{\phi}_{t}, \frac{\alpha \xi}{\beta} \frac{\partial\left(\mathbf{I}-D \gamma_{0}\right) \widehat{\phi}}{\partial \nu}+\frac{\alpha}{\beta} \frac{\partial\left(\mathbf{I}-D \gamma_{0}\right) \widehat{\phi}_{t}}{\partial \nu}\right)_{L^{2}\left(\Gamma_{1}\right)} d t+\frac{c_{1}}{\gamma} \int_{s}^{\tau} a\left(\widehat{\phi}, A_{D}^{-1} \widehat{\psi}\right)_{L^{2}(\Omega)} d t \\
& +\frac{c_{1}}{\gamma} \int_{s}^{\tau}\left[\left(\alpha \widehat{\psi}, \frac{\partial A_{D}^{-1} \widehat{\psi}}{\partial \nu}\right)_{L^{2}\left(\Gamma_{1}\right)}-\left(\Delta \widehat{\phi}, \frac{\partial A_{D}^{-1} \widehat{\psi}}{\partial \nu}\right)_{L^{2}\left(\Gamma_{0}\right)} d t+\frac{c_{1}}{\gamma}\left[\left(\widehat{\phi}_{t}, A_{D}^{-1} \widehat{\psi}\right)_{L^{2}(\Omega)}\right]_{s}^{\tau}\right.
\end{aligned}
$$

(note the cancellation of the high order term $\int_{s}^{\tau}\left(\widehat{\psi}_{t}, \widehat{\phi}_{t}\right)_{L^{2}(\Omega)} d t$ ).

We now proceed to estimate the right-hand side of this relation. In so doing, we will be using implicitly, in (B1)-(B7) below, the inequality $a b \leq \epsilon a^{2}+C_{\epsilon} b^{2}$. 
(B1) We have by trace theory

$$
\begin{aligned}
-\int_{s}^{\tau}\left[\left(\gamma \xi^{2} \frac{\partial \widehat{\phi}}{\partial \nu}+2 \gamma \xi \frac{\partial \widehat{\phi}_{t}}{\partial \nu}+\alpha \lambda \gamma_{0} \widehat{\psi}, \widehat{\phi}_{t}\right)_{L^{2}\left(\Gamma_{1}\right)}+\alpha\left(\widehat{\psi}, \frac{\partial \widehat{\phi}_{t}}{\partial \nu}\right)_{L^{2}\left(\Gamma_{1}\right)}\right] d t \\
(2.27) \leq C \int_{0}^{T}\left\|\frac{\partial \widehat{\phi}_{t}}{\partial \nu}\right\|_{L^{2}\left(\Gamma_{1}\right)}^{2} d t+\text { l.o.t. }\left(\widehat{\phi}, \widehat{\phi}_{t}, \widehat{\psi}\right) .
\end{aligned}
$$

(B2) As $A_{D}^{-1}$ is a bounded operator, we have

$$
\begin{aligned}
& \int_{s}^{\tau}\left(c_{0} \widehat{\psi}+c_{2} \widehat{\phi}+c_{3} \widehat{\phi}_{t}+c_{4} \Delta \widehat{\phi}, \widehat{\phi}_{t}-\frac{c_{1}}{\gamma} A_{D}^{-1} \widehat{\psi}\right) d t \\
\leq & \frac{\epsilon}{6} \int_{0}^{T}\left\|\AA^{\frac{1}{2}} \widehat{\phi}\right\|_{L^{2}(\Omega)}^{2} d t+\text { I.o.t. }\left(\widehat{\phi}, \widehat{\phi}_{t}, \widehat{\psi}\right) .
\end{aligned}
$$

(B3) As $D \gamma_{0} \in \mathcal{L}\left(H^{s}(\Omega)\right)$ for $s>\frac{1}{2}$ (by standard elliptic theory), and $A_{D}^{-1}$ $\in \mathcal{L}\left(L^{2}(\Omega), D\left(A_{D}\right)\right)$, we then have in conjunction with trace theory

$$
\begin{aligned}
& -\frac{c_{1}}{\gamma} \int_{s}^{\tau}\left(\widehat{\phi}_{t},\left(\frac{\sigma}{\beta}-\xi\right) A_{D}^{-1} \widehat{\psi}+\frac{\eta}{\beta}\left(\mathbf{I}-D \gamma_{0}\right) \widehat{\psi}\right)_{L^{2}(\Omega)} d t \\
& +\frac{c_{1}}{\gamma} \int_{s}^{\tau}\left(\widehat{\phi}_{t}, \frac{\alpha \xi}{\beta}\left(\mathbf{I}-D \gamma_{0}\right) \widehat{\phi}+\frac{\alpha}{\beta}\left(\mathbf{I}-D \gamma_{0}\right) \widehat{\phi}_{t}\right)_{L^{2}(\Omega)} d t \\
& -c_{1} \int_{s}^{\tau}\left(\widehat{\phi}_{t},\left(\frac{\sigma}{\beta}-\xi\right) \frac{\partial A_{D}^{-1} \widehat{\psi}}{\partial \nu}\right)_{L^{2}\left(\Gamma_{1}\right)} d t \\
& +\frac{c_{1}}{\gamma} \int_{s}^{\tau} a\left(\widehat{\phi}, A_{D}^{-1} \widehat{\psi}\right) d t+\frac{\alpha c_{1}}{\gamma} \int_{s}^{\tau}\left(\widehat{\psi}, \frac{\partial A_{D}^{-1} \widehat{\psi}}{\partial \nu}\right)_{L^{2}\left(\Gamma_{1}\right)} d t \\
& \leq \frac{\epsilon}{6} \int_{0}^{T}\left\|\AA^{\frac{1}{2}} \widehat{\phi}\right\|_{L^{2}(\Omega)}^{2} d t+\text { I.o.t. }\left(\widehat{\phi}, \widehat{\phi}_{t}, \widehat{\psi}\right) .
\end{aligned}
$$

(B4) Using the fact that $D \gamma_{0} \in \mathcal{L}\left(H^{s}(\Omega)\right)$ for $s>\frac{1}{2}$, and $\left.\frac{\partial \widehat{\psi}}{\partial \nu}(t)\right|_{\Gamma}=-\left.\lambda \widehat{\psi}(t)\right|_{\Gamma}$, we have along with trace theory that

$$
\begin{aligned}
& c_{1} \int_{s}^{\tau}\left(\widehat{\phi}_{t},-\frac{\eta}{\beta} \frac{\partial \widehat{\psi}}{\partial \nu}+\frac{\alpha \xi}{\beta} \frac{\partial\left(\mathbf{I}-D \gamma_{0}\right) \widehat{\phi}}{\partial \nu}+\frac{\alpha}{\beta} \frac{\partial \widehat{\phi}_{t}}{\partial \nu}\right)_{L^{2}\left(\Gamma_{1}\right)} d t \\
\leq & C \int_{0}^{T}\left\|\frac{\partial \widehat{\phi}_{t}}{\partial \nu}\right\|_{L^{2}\left(\Gamma_{1}\right)}^{2} d t+\text { l.o.t. }\left(\widehat{\phi}, \widehat{\phi}_{t}, \widehat{\psi}\right) .
\end{aligned}
$$

(B5) By [1, p. 311, Theorem 3] and trace theory we deduce that $\frac{\partial}{\partial \nu} D \gamma_{0} \in$ $\mathcal{L}\left(H^{1}(\Omega), H^{-\frac{1}{2}}(\Gamma)\right)$, and so accordingly we have

$$
\begin{aligned}
& c_{1} \int_{s}^{\tau}\left(\widehat{\phi}_{t}, \frac{\eta}{\beta} \frac{\partial D \gamma_{0} \widehat{\psi}}{\partial \nu}-\frac{\alpha}{\beta} \frac{\partial D \gamma_{0} \widehat{\phi}_{t}}{\partial \nu}\right)_{L^{2}\left(\Gamma_{1}\right)} d t \leq C_{\epsilon} \int_{0}^{T}\left\|\widehat{\phi}_{t}\right\|_{H^{\frac{1}{2}\left(\Gamma_{1}\right)}}^{2} d t \\
& \quad+\frac{\epsilon}{6} \int_{0}^{T}\left[\left\|P_{\gamma}^{\frac{1}{2}} \widehat{\phi}_{t}\right\|_{L^{2}(\Omega)}^{2}+\left\|A_{R}^{\frac{1}{2}} \widehat{\psi}\right\|_{L^{2}(\Omega)}^{2}\right] d t .
\end{aligned}
$$


(B6) As $A_{D}^{-1} \in \mathcal{L}\left(H^{-1}(\Omega), H_{0}^{1}(\Omega)\right)$, by the characterizations of elliptic operators given in $[7]$, we then have for all $t \in[0, T]$

$$
\begin{aligned}
\left(\nabla \widehat{\phi}_{t}(t), \nabla A_{D}^{-1} \widehat{\psi}(t)\right)_{L^{2}(\Omega)} & \leq C\left\|\nabla \widehat{\phi}_{t}(t)\right\|_{L^{2}(\Omega)}\left\|\nabla A_{D}^{-1} \widehat{\psi}(t)\right\|_{L^{2}(\Omega)} \\
& \leq \frac{\epsilon}{6}\left\|P_{\gamma}^{\frac{1}{2}} \widehat{\phi}_{t}(t)\right\|_{L^{2}(\Omega)}^{2}+\text { I.o.t. }\left(\widehat{\phi}, \widehat{\phi}_{t}, \widehat{\psi}\right) .
\end{aligned}
$$

We thus have

$$
\begin{aligned}
& c_{1}\left[\left(\nabla \widehat{\phi}_{t}(t), \nabla A_{D}^{-1} \widehat{\psi}\right)_{L^{2}(\Omega)}\right]_{t=s}^{t=\tau}+\left[\frac{c_{1}}{\gamma}\left(\widehat{\phi}_{t}(t), A_{D}^{-1} \widehat{\psi}(t)\right)_{L^{2}(\Omega)}\right]_{t=s}^{t=\tau} \\
\leq & \frac{\epsilon}{6}\left(\left\|P_{\gamma}^{\frac{1}{2}} \widehat{\phi}_{t}(\tau)\right\|_{L^{2}(\Omega)}^{2}+\left\|P_{\gamma}^{\frac{1}{2}} \widehat{\phi}_{t}(s)\right\|_{L^{2}(\Omega)}^{2}\right)+\text { I.o.t. }\left(\widehat{\phi}, \widehat{\phi}_{t}, \widehat{\psi}\right) .
\end{aligned}
$$

(B7) Finally, we can use the trace result Lemma 4.5 of the appendix and the fact that $A_{D}^{-1} \in \mathcal{L}\left(H^{-\frac{1}{2}+\epsilon}(\Omega), H^{\frac{3}{2}+\epsilon}(\Omega)\right.$ (again by [7]) to have

$$
\begin{aligned}
& -\frac{c_{1}}{\gamma} \int_{s}^{\tau}\left(\Delta \widehat{\phi}, \frac{\partial A_{D}^{-1} \widehat{\psi}}{\partial \nu}\right)_{L^{2}\left(\Gamma_{0}\right)} d t \\
\leq & C \int_{s}^{\tau}\|\Delta \widehat{\phi}\|_{L^{2}\left(\Gamma_{0}\right)}\|\widehat{\psi}\|_{H^{-\frac{1}{2}+\epsilon}(\Omega)} d t \leq \frac{\epsilon}{6 C_{0}} \int_{s}^{\tau}\|\Delta \widehat{\phi}\|_{L^{2}\left(\Gamma_{0}\right)}^{2} d t+C_{\epsilon} \int_{0}^{T}\|\widehat{\psi}\|_{H^{-\frac{1}{2}+\epsilon}(\Omega)}^{2} d t
\end{aligned}
$$

(where the constant $C_{0}$ above is the very same as that in (4.13))

$\leq \frac{\epsilon}{3} \int_{0}^{T} E_{\widehat{\phi}}(t) d t+\frac{\epsilon}{3}\left[E_{\widehat{\phi}}(s)+E_{\widehat{\phi}}(\tau)\right]+$ l.o.t. $\left(\widehat{\phi}, \widehat{\phi}_{t}, \widehat{\psi}\right)$.

Therefore, if we define $\mathcal{F}(s, \tau)$ to be

$$
\mathcal{F}(s, \tau) \equiv \text { right-hand side of }(2.26),
$$

estimates (2.27)-(2.33), then we have

$$
\begin{aligned}
\mathcal{F}(s, \tau) & \leq C_{\epsilon} \int_{0}^{T}\left\|\nabla \widehat{\phi}_{t}\right\|_{L^{2}\left(\Gamma_{1}\right)}^{2} d t+\epsilon \int_{0}^{T}\left[E_{\widehat{\phi}}(t)+\left\|A_{R}^{\frac{1}{2}} \widehat{\psi}(t)\right\|_{L^{2}(\Omega)}^{2}\right] d t \\
& +\epsilon\left[E_{\widehat{\phi}}(s)+E_{\widehat{\phi}}(\tau)\right]+\text { l.o.t. }\left(\widehat{\phi}, \widehat{\phi}_{t}, \widehat{\psi}\right)
\end{aligned}
$$

where the constant $C_{\epsilon}$ does not depend on time $T$. This and equality (2.26) prove (a).

To prove (b), we combine (2.16) and (2.17) and subsequently take $\epsilon>0$ small enough. The proof of Lemma 2.2 is concluded.

With the radial vector field $\bar{h}$ defined in (1.3), one has the following relation, which is essentially demonstrated in [12] (the complete proof is carried out in Proposition 4.6 of the appendix below).

Proposition 2.3. With the vector field $\bar{h}$ as defined in (1.3), the solution $\left[\widehat{\phi}, \widehat{\phi}_{t}, \widehat{\psi}\right]$ to $(2.15)$, corresponding to terminal data $\left[\phi_{0}, \phi_{1}, \psi_{0}\right] \in D\left(\left[\mathcal{A}_{\gamma}^{*}\right]^{2}\right)$, satisfies the following equality for arbitrary $\epsilon_{0} \in[0, T)$ : 


$$
\begin{aligned}
& \int_{\epsilon_{0}}^{T-\epsilon_{0}} E_{\widehat{\phi}}(t) d t=\int_{\epsilon_{0}}^{T-\epsilon_{0}}\left(c_{0} \widehat{\psi}+c_{2} \widehat{\phi}+c_{3} \widehat{\phi}_{t}+c_{4} \Delta \widehat{\phi}, \bar{h} \cdot \nabla \widehat{\phi}-\frac{1}{2} \widehat{\phi}\right)_{L^{2}(\Omega)} d t \\
& -c_{1} \int_{\epsilon_{0}}^{T-\epsilon_{0}}\left(\widehat{\psi}, \bar{h} \cdot \nabla \widehat{\phi}_{t}-\frac{1}{2} \widehat{\phi}_{t}\right)_{L^{2}(\Omega)} d t+\frac{1}{2} \int_{\epsilon_{0}}^{T-\epsilon_{0}} \int_{\Gamma_{1}} \bar{h} \cdot \nu\left(\widehat{\phi}_{t}^{2}+\gamma\left|\nabla \widehat{\phi}_{t}\right|^{2}\right) d \Gamma d t \\
& -\int_{\epsilon_{0}}^{T-\epsilon_{0}}\left\|\widehat{\phi}_{t}\right\|_{L^{2}(\Omega)}^{2} d t+\frac{1}{2} \int_{\epsilon_{0}}^{T-\epsilon_{0}} \int_{\Gamma_{0}} \bar{h} \cdot \nu(\Delta \widehat{\phi})^{2} d \Gamma d t \\
& -\int_{\epsilon_{0}}^{T-\epsilon_{0}}\left[\alpha\left(\widehat{\psi}, \frac{\partial}{\partial \nu}\left(\bar{h} \cdot \nabla \widehat{\phi}-\frac{1}{2} \widehat{\phi}\right)\right)_{L^{2}\left(\Gamma_{1}\right)}\right. \\
& \left.-\left[\gamma \frac{\partial}{\partial \nu}\left(\xi^{2} \widehat{\phi}+2 \xi \widehat{\phi} t\right)-\alpha \frac{\partial \hat{\psi}}{\partial \nu}, \bar{h} \cdot \nabla \widehat{\phi}-\frac{1}{2} \widehat{\phi}\right)_{L^{2}\left(\Gamma_{1}\right)}\right] d t \\
& +\left[\frac{\gamma}{2}(\nabla \widehat{\phi} t, \nabla \widehat{\phi})_{L^{2}(\Omega)}+c_{1}\left(\widehat{\psi}, \bar{h} \cdot \nabla \widehat{\phi}-\frac{1}{2} \widehat{\phi}\right)_{L^{2}(\Omega)}\right]_{t=\epsilon_{0}}^{t=T-\epsilon_{0}} \\
& -\int_{\epsilon_{0}}^{T-\epsilon_{0}} \int_{\Gamma_{1}} \frac{\bar{h} \cdot \nu}{2}\left[\left(\frac{\partial^{2} \widehat{\phi}}{\partial x^{2}}\right)^{2}+\left(\frac{\partial^{2} \widehat{\phi}}{\partial y^{2}}\right)^{2}\right. \\
& \left.+2 \mu\left(\frac{\partial^{2} \widehat{\phi}}{\partial x^{2}}\right)\left(\frac{\partial^{2} \widehat{\phi}}{\partial y^{2}}\right)+2(1-\mu)\left(\frac{\partial^{2} \widehat{\phi}}{\partial x \partial y}\right)^{2}\right] d t d \Gamma .
\end{aligned}
$$

So as to derive another intermediate energy inequality, we will now estimate the right-hand side of the relation (2.36). In the course of this work, we will make critical use of the following trace estimate for (uncoupled ) Kirchoff plates, which was derived in [15]. It is this regularity result that allows the controlled portion $\Gamma_{1}$ of the boundary to be free of geometric constraints.

Trace theOrem (see [15]). Let the function $\varphi(t, x)$ satisfy the following Kirchoff equation on an open, bounded domain $\Omega \subset \mathbb{R}^{n}$, with smooth boundary $\Gamma, \Gamma=\Gamma_{0} \cup \Gamma_{1}$, where each $\Gamma_{i}$ is open and nonempty, with $\bar{\Gamma}_{0} \cap \bar{\Gamma}_{1}=\emptyset$ :

$$
\left\{\begin{array}{l}
\varphi_{t t}-\gamma \Delta \varphi_{t t}+\Delta^{2} \varphi=f \quad \text { on }(0, T) \times \Omega \\
\varphi=\frac{\partial \varphi}{\partial \nu}=0 \text { on }(0, T) \times \Gamma_{0} \\
\left\{\begin{array}{l}
\Delta \varphi+(1-\mu) B_{1} \varphi=g_{1} \\
\frac{\partial \Delta \varphi}{\partial \nu}+(1-\mu) \frac{\partial B_{2} \varphi}{\partial \tau}-\gamma \frac{\partial \varphi_{t t}}{\partial \nu}=g_{2}
\end{array} \text { on }(0, T) \times \Gamma_{1}\right.
\end{array}\right.
$$

(here the boundary operators $B_{1}$ and $B_{2}$ are as given in (1.2)). Let $0<\epsilon_{0}<\frac{T}{2}$ and $\epsilon>0$ be arbitrary. Then the following inequality holds true for the solution $\varphi$ :

$$
\int_{\epsilon_{0}}^{T-\epsilon_{0}}\left[\left\|\frac{\partial^{2} \varphi}{\partial \tau^{2}}\right\|_{L^{2}\left(\Gamma_{1}\right)}^{2}+\left\|\frac{\partial^{2} \varphi}{\partial \nu^{2}}\right\|_{L^{2}\left(\Gamma_{1}\right)}^{2}+\left\|\frac{\partial^{2} \varphi}{\partial \tau \partial \nu}\right\|_{L^{2}\left(\Gamma_{1}\right)}^{2}\right] d t
$$




$$
\begin{aligned}
\leq C_{T, \epsilon_{0}, \gamma}\{ & {\left[\int_{0}^{T}\|f\|_{\left[H^{\frac{3}{2}-\epsilon}(\Omega)\right]^{\prime}}^{2}+\left\|g_{1}\right\|_{L^{2}\left(\Gamma_{1}\right)}^{2}+\|\varphi\|_{H^{\frac{3}{2}+\epsilon}\left(\Gamma_{1}\right)}^{2}+\left\|\left|\nabla \varphi_{t}\right|\right\|_{L^{2}\left(\Gamma_{1}\right)}^{2}\right.} \\
& \left.\left.+\left\|\varphi_{t}\right\|_{L^{2}\left(\Gamma_{1}\right)}^{2}\right] d t+\left\|g_{2}\right\|_{H^{-1}\left(0, T \times \Gamma_{1}\right)}^{2}\right\} .
\end{aligned}
$$

Remark 2.4. In the original statement of this theorem (see Theorem 2.1 in [15]), the term $\int_{0}^{T}\|f\|_{\left[H^{\frac{3}{2}-\epsilon}(\Omega)\right]^{\prime}}^{2} d t$ in the inequality (2.38) is replaced by $\|f\|_{\left[H^{q}(0, T \times \Omega)\right]^{\prime}}^{2}$, where $q<\frac{1}{2}$. However, if one replaces the $H^{-q}(0, T \times \Omega)$ spaces with $L^{2}\left(0, T ;\left[H^{q}(\Omega)\right]^{\prime}\right)$, the values of allowed parameters extend to $q<3 / 2+\epsilon$. This is in line with elliptic theory corresponding to free boundary conditions.

By the use of this trace result in part, we have the following energy estimate.

Lemma 2.5. For all $\epsilon_{0} \in\left(0, \frac{T}{2}\right)$ and $\widetilde{\epsilon}>0$ arbitrary, the solution $\left[\widehat{\phi}, \widehat{\phi}_{t}, \widehat{\psi}\right]$ to (2.15) satisfies

$$
\begin{aligned}
& \int_{\epsilon_{0}}^{T-\epsilon_{0}} E_{\widehat{\phi}}(t) d t \leq C^{*}\left(E_{\widehat{\phi}}\left(T-\epsilon_{0}\right)+E_{\widehat{\phi}}\left(\epsilon_{0}\right)\right) \\
+ & C_{T} \int_{0}^{T}\left\|\nabla \widehat{\phi}_{t}\right\|_{L^{2}\left(\Gamma_{1}\right)}^{2}+\widetilde{\epsilon} \int_{0}^{T}\left\|A_{R}^{\frac{1}{2}} \widehat{\psi}\right\|_{L^{2}(\Omega)}^{2}+\text { l.o.t. }\left(\widehat{\phi}, \widehat{\phi}_{t}, \widehat{\psi}\right),
\end{aligned}
$$

where the (time independent) constant $C^{*} \geq \sqrt{\frac{2 \gamma}{1-\mu}} \max _{[x, y] \in \bar{\Omega}}|\bar{h}(x, y)|$ (where, again, $\mu$ is Poisson's ratio and $\bar{h}$ satisfies 1.3 ).

Proof. We proceed to majorize the right-hand side of (2.36).

(A.1) Handling the term $\int_{\epsilon_{0}}^{T-\epsilon_{0}}\left(c_{0} \widehat{\psi}+c_{2} \widehat{\phi}+c_{3} \widehat{\phi}_{t}+c_{4} \Delta \widehat{\phi}, \bar{h} \cdot \nabla \widehat{\phi}-\frac{1}{2} \widehat{\phi}\right)_{L^{2}(\Omega)} d t$ : First, by Green's theorem and the fact that $\nabla \in \mathcal{L}\left(H^{s}(\Omega), H^{s-1}(\Omega)\right)$ and $\nabla(\bar{h} \cdot \nabla) \in$ $\mathcal{L}\left(H^{s}(\Omega), H^{s-2}(\Omega)\right)$, we obtain

$$
\begin{aligned}
&\left(\Delta \widehat{\phi}, \bar{h} \cdot \nabla \widehat{\phi}-\frac{1}{2} \widehat{\phi}\right)_{L^{2}(\Omega)}=-\left(\nabla \widehat{\phi}, \nabla(\bar{h} \cdot \nabla \widehat{\phi})-\frac{1}{2} \widehat{\nabla \phi}\right)_{L^{2}(\Omega)} \\
&+\left(\frac{\partial \widehat{\phi}}{\partial \nu}, \nabla \widehat{\phi}-\frac{1}{2} \widehat{\phi}\right)_{L^{2}\left(\Gamma_{1}\right)} \\
&=-\left(\nabla \widehat{\phi}, \nabla(\bar{h} \cdot \nabla \widehat{\phi})-\frac{1}{2} \widehat{\nabla \phi}\right)_{H^{\epsilon}(\Omega) \times H^{-\epsilon}(\Omega)}+\left(\frac{\partial \hat{\phi}}{\partial \nu}, \nabla \widehat{\phi}-\frac{1}{2} \widehat{\phi}\right)_{L^{2}\left(\Gamma_{1}\right)} \\
& \text { < I.o.t. }\left(\widehat{\phi}, \widehat{\phi}_{t}, \widehat{\psi}\right),
\end{aligned}
$$

where in the last step we have also used Cauchy-Schwarz and the trace theory. We thus have

$$
\int_{\epsilon_{0}}^{T-\epsilon_{0}}\left(c_{0} \widehat{\psi}+c_{2} \widehat{\phi}+c_{3} \widehat{\phi}_{t}+c_{4} \Delta \widehat{\phi}, \bar{h} \cdot \nabla \widehat{\phi}-\frac{1}{2} \widehat{\phi}\right)_{L^{2}(\Omega)} d t \leq \text { I.o.t. }\left(\widehat{\phi}, \widehat{\phi}_{t}, \widehat{\psi}\right) .
$$

(A.2) Likewise using Sobolev trace theory, the fact that $\frac{\partial \widehat{\psi}}{\partial \nu}=-\lambda \widehat{\psi}$, and the divergence theorem, we have 


$$
\begin{aligned}
& -\int_{\epsilon_{0}}^{T-\epsilon_{0}}\left[c_{1}\left(\widehat{\psi}, \bar{h} \cdot \nabla \widehat{\phi}_{t}-\frac{1}{2} \widehat{\phi}_{t}\right)_{L^{2}(\Omega)}+\left\|\widehat{\phi}_{t}\right\|_{L^{2}(\Omega)}^{2}\right] d t \\
& +\int_{\epsilon_{0}}^{T-\epsilon_{0}}\left[-\left(\gamma \frac{\partial}{\partial \nu}\left(\xi^{2} \widehat{\phi}+2 \xi \widehat{\phi}_{t}\right)-\alpha \frac{\partial \widehat{\psi}}{\partial \nu}, \bar{h} \cdot \nabla \widehat{\phi}-\frac{1}{2} \widehat{\phi}\right)_{L^{2}\left(\Gamma_{1}\right)}+\frac{\alpha}{2}\left(\widehat{\psi}, \frac{\partial \widehat{\phi}}{\partial \nu}\right)_{L^{2}\left(\Gamma_{1}\right)}\right] d t \\
\leq & c_{1} \int_{\epsilon_{0}}^{T-\epsilon_{0}}\left(\widehat{\psi}, h_{1} \widehat{\phi}_{t x}+h_{2} \widehat{\phi}_{t y}\right)_{L^{2}(\Omega)} d t+C \int_{0}^{T}\left\|\nabla \widehat{\phi}_{t}\right\|_{L^{2}\left(\Gamma_{1}\right)}^{2} d t+\text { I.o.t. }\left(\widehat{\phi}, \widehat{\phi}_{t}, \widehat{\psi}\right) \\
= & -c_{1} \int_{\epsilon_{0}}^{T-\epsilon_{0}} \int_{\Omega} \operatorname{div}(\widehat{\psi} h) \widehat{\phi}_{t} d \Omega d t \\
& +c_{1} \int_{\epsilon_{0}}^{T-\epsilon_{0}} \int_{\Gamma_{1}} \bar{h} \cdot \nu \widehat{\psi} \widehat{\phi}_{t} d \Gamma d t+C \int_{0}^{T}\left\|\nabla \widehat{\phi}_{t}\right\|_{L^{2}\left(\Gamma_{1}\right)}^{2} d t+\text { I.o.t. }(\widehat{\phi}, \widehat{\phi} t, \widehat{\psi}) \\
\leq & C \int_{0}^{T}\left\|\nabla \widehat{\phi}_{t}\right\|_{L^{2}\left(\Gamma_{1}\right)}^{2} d t+\widetilde{\epsilon} \int_{0}^{T}\left\|A_{R}^{\frac{1}{2}} \widehat{\psi}\right\|_{L^{2}(\Omega)}^{2}+\text { I.o.t. }\left(\widehat{\phi}, \widehat{\phi}_{t}, \widehat{\psi}\right) .
\end{aligned}
$$

(A.3) Using (1.3), we have

$$
\frac{1}{2} \int_{\epsilon_{0}}^{T-\epsilon_{0}} \int_{\Gamma_{0}} \bar{h} \cdot \nu(\Delta \widehat{\phi})^{2} \leq 0
$$

(A.4) We now estimate the terms

$$
\begin{aligned}
- & {\left[\left(\widehat{\phi}_{t}, \bar{h} \cdot \nabla \widehat{\phi}\right)_{L^{2}(\Omega)}+\gamma\left(\nabla \widehat{\phi}_{t}, \nabla(\bar{h} \cdot \nabla \widehat{\phi})\right)_{L^{2}(\Omega)}-\frac{1}{2}\left(\widehat{\phi}_{t}, \widehat{\phi}\right)_{L^{2}(\Omega)}\right]_{t=\epsilon_{0}}^{t=T-\epsilon_{0}} } \\
(2.43)+ & {\left[\frac{\gamma}{2}\left(\nabla \widehat{\phi}_{t}, \nabla \widehat{\phi}\right)_{L^{2}(\Omega)}+c_{1}\left(\widehat{\psi}, \bar{h} \cdot \nabla \widehat{\phi}-\frac{1}{2} \widehat{\phi}\right)_{L^{2}(\Omega)}\right]_{t=\epsilon_{0}}^{t=T-\epsilon_{0}} }
\end{aligned}
$$

First, as $\bar{h} \cdot \nabla \widehat{\phi}(t) \in H^{\frac{1}{2}-\epsilon}(\Omega)$ for all $t \in[0, T]$, we have

$$
\begin{aligned}
& \left(\widehat{\psi}(t), \bar{h} \cdot \nabla \widehat{\phi}(t)-\frac{1}{2} \widehat{\phi}(t)\right)_{L^{2}(\Omega)}=\left\langle\widehat{\psi}(t), \bar{h} \cdot \nabla \widehat{\phi}(t)-\frac{1}{2} \widehat{\phi}(t)\right\rangle_{H^{-\frac{1}{2}+\epsilon}(\Omega) \times H^{\frac{1}{2}-\epsilon}(\Omega)} \\
\leq & \text { I.o.t. }\left(\widehat{\phi}, \widehat{\phi}_{t}, \widehat{\psi}\right) .
\end{aligned}
$$

Second, we have pointwise in time

$$
\begin{aligned}
& \gamma\left(\nabla \widehat{\phi}_{t}, \nabla(\bar{h} \cdot \nabla \widehat{\phi})\right)_{L^{2}(\Omega)}+\frac{\gamma}{2}\left(\nabla \widehat{\phi}_{t}, \nabla \widehat{\phi}\right)_{L^{2}(\Omega)} \\
&= \sqrt{\gamma} \int_{\Omega} \sqrt{\gamma} \nabla \widehat{\phi}_{t}(x, y) \cdot\left[\left(x-x_{0}\right) \phi_{x x}+\left(y-y_{0}\right) \phi_{x y},\left(x-x_{0}\right) \phi_{x y}+\left(y-y_{0}\right) \phi_{y y}\right] d x d y \\
&(2.45) \quad+\frac{3 \gamma}{2}\left(\nabla \widehat{\phi}_{t}, \nabla \widehat{\phi}\right)_{L^{2}(\Omega)} .
\end{aligned}
$$

Now, to handle the first term on the right-hand side of (2.45), we use the inequality $a b \leq \frac{\delta}{2} a^{2}+\frac{1}{2 \delta} b^{2}$ with $\delta \equiv \sqrt{2(1-\mu)}$ (where, again, Poisson's ratio $\left.\mu \in\left(0, \frac{1}{2}\right)\right)$ 


$$
\begin{gathered}
\int_{\Omega} \sqrt{\gamma} \nabla \widehat{\phi}_{t}(x, y) \cdot\left[\left(x-x_{0}\right) \phi_{x x}+\left(y-y_{0}\right) \phi_{x y},\left(x-x_{0}\right) \phi_{x y}+\left(y-y_{0}\right) \phi_{y y}\right] d x d y \\
=\int_{\Omega}\left(x-x_{0}\right) \sqrt{\gamma} \nabla \widehat{\phi}_{t} \cdot\left[\phi_{x x}, \phi_{x y}\right] d x d y+\int_{\Omega}\left(y-y_{0}\right) \sqrt{\gamma} \nabla \widehat{\phi}_{t} \cdot\left[\phi_{x y}, \phi_{y y}\right] d x d y \\
\leq \max _{[x, y] \in \bar{\Omega}}|\bar{h}(x, y)|\left\{\frac{\gamma}{\sqrt{2(1-\mu)}} \int_{\Omega}\left|\nabla \phi_{t}\right|^{2} d \Omega+\frac{\sqrt{(1-\mu)}}{\sqrt{2}} \int_{\Omega}\left[\phi_{x x}^{2}+\phi_{y y}^{2}\right] d \Omega\right. \\
\left.+\sqrt{2(1-\mu)} \int_{\Omega} \phi_{x y}^{2} d \Omega\right\} \\
\leq \frac{1}{\sqrt{2(1-\mu)}} \max _{[x, y] \in \bar{\Omega}}|\bar{h}(x, y)|\left\{\left\|P_{\gamma}^{\frac{1}{2}} \widehat{\phi}_{t}\right\|_{L^{2}(\Omega)}^{2}+(1-\mu) \int_{\Omega}\left[\phi_{x x}^{2}+\phi_{y y}^{2}\right] d \Omega\right. \\
\left.\quad+2(1-\mu) \int_{\Omega} \phi_{x y}^{2} d \Omega\right\} .
\end{gathered}
$$

From this inequality, the definition of $a(\cdot, \cdot)$ in (1.10), and the characterization in (1.11), we obtain

$$
\begin{aligned}
& \int_{\Omega} \sqrt{\gamma} \nabla \widehat{\phi}_{t}(x, y) \cdot\left[\left(x-x_{0}\right) \phi_{x x}+\left(y-y_{0}\right) \phi_{x y},\left(x-x_{0}\right) \phi_{x y}+\left(y-y_{0}\right) \phi_{y y}\right] d x d y \\
\leq & \frac{1}{\sqrt{2(1-\mu)}} \max _{[x, y] \in \bar{\Omega}}|\bar{h}(x, y)|\left\{\left\|P_{\gamma}^{\frac{1}{2}} \widehat{\phi}_{t}\right\|_{L^{2}(\Omega)}^{2}+\left\|\AA^{\frac{1}{2}} \widehat{\phi}\right\|_{L^{2}(\Omega)}^{2}\right\} .
\end{aligned}
$$

To deal with the second term on the right-hand side of (2.45), we can use the fact that $\nabla \in \mathcal{L}\left(H^{s}(\Omega), H^{1-s}(\Omega)\right)$ for all real $s$, so as to have

$$
\begin{aligned}
& \left(\nabla \widehat{\phi}_{t}(t), \nabla \widehat{\phi}(t)\right)_{L^{2}(\Omega)}=\left\langle\nabla \widehat{\phi}_{t}(t), \nabla \widehat{\phi}(t)\right\rangle_{H^{-\epsilon}(\Omega) \times H^{\epsilon}(\Omega)} \\
\leq & C\left\|\widehat{\phi}_{t}(t)\right\|_{H^{1-\epsilon}(\Omega)}\|\widehat{\phi}(t)\|_{H^{1+\epsilon}(\Omega} \leq \text { I. o.t. }\left(\widehat{\phi}, \widehat{\phi}_{t}, \widehat{\psi}\right) .
\end{aligned}
$$

Combining (2.45), (2.47), and (2.48) with the definition of $E_{\widehat{\phi}}$ in (2.3), we then obtain

$$
\begin{aligned}
& \left.\gamma\left(\nabla \widehat{\phi}_{t}(t), \nabla(\bar{h} \cdot \nabla \widehat{\phi}(t))\right)\right)_{L^{2}(\Omega)}+\frac{\gamma}{2}\left(\nabla \widehat{\phi}_{t}(t), \nabla \widehat{\phi}(t)\right)_{L^{2}(\Omega)} \\
\leq & \sqrt{\frac{2 \gamma}{1-\mu}} \max _{(x, y) \in \bar{\Omega}}|\bar{h}(x, y)| E_{\widehat{\phi}}(t)+\text { l.o.t. }\left(\widehat{\phi}, \widehat{\phi}_{t}, \widehat{\psi}\right) .
\end{aligned}
$$

Coupling (2.44) and (2.49) in turn, we arrive at the estimate

$(2.43) \leq \sqrt{\frac{2 \gamma}{1-\mu}}\left\{\max _{(x, y) \in \bar{\Omega}}|\bar{h}(x, y)|\right\}\left(E_{\widehat{\phi}}\left(T-\epsilon_{0}\right)+E_{\widehat{\phi}}\left(\epsilon_{0}\right)\right)+$ l.o.t. $\left(\widehat{\phi}, \widehat{\phi}_{t}, \widehat{\psi}\right)$.

(A.5) Handling the term $-\int_{\epsilon_{0}}^{T-\epsilon_{0}} \alpha\left(\widehat{\psi}, \frac{\partial}{\partial \nu}(\bar{h} \cdot \nabla \widehat{\phi})\right)$ and noting that

$$
\begin{aligned}
\frac{\partial}{\partial \nu}(\bar{h} \cdot \nabla \hat{\phi})= & \nu_{1} \widehat{\phi}_{x}+\nu_{1}\left(x-x_{0}\right) \widehat{\phi}_{x x}+\nu_{1}\left(y-y_{0}\right) \widehat{\phi}_{x y}+\nu_{2}\left(x-x_{0}\right) \widehat{\phi}_{x y} \\
& +\nu_{2} \widehat{\phi}_{y}+\nu_{2}\left(y-y_{0}\right) \widehat{\phi}_{y y}
\end{aligned}
$$


we then have by Cauchy-Schwarz, the trace estimate (2.38) for the Kirchoff plates above, the use of the forcing data in (2.15), and the standard Sobolev trace theory that

$$
\begin{aligned}
& -\int_{\epsilon_{0}}^{T-\epsilon_{0}} \alpha\left(\widehat{\psi}, \frac{\partial}{\partial \nu}(\bar{h} \cdot \nabla \widehat{\phi})\right)_{L^{2}\left(\Gamma_{1}\right)} d t \\
& \leq C \int_{\epsilon_{0}}^{T-\epsilon_{0}}\left[\|\widehat{\psi}\|_{H^{\frac{1}{2}+\epsilon}(\Omega)}^{2}+\left\|\widehat{\phi}_{x x}\right\|_{L^{2}\left(\Gamma_{1}\right)}^{2}+\left\|\widehat{\phi}_{y y}\right\|_{L^{2}\left(\Gamma_{1}\right)}^{2}+2\left\|\widehat{\phi}_{x y}\right\|_{L^{2}\left(\Gamma_{1}\right)}^{2}\right. \\
& \left.+\left\|\widehat{\phi}_{x}\right\|_{H^{\frac{1}{2}+\epsilon}(\Omega)}^{2}+\left\|\widehat{\phi}_{y}\right\|_{H^{\frac{1}{2}+\epsilon}(\Omega)}^{2}\right] d t \\
& =C \int_{\epsilon_{0}}^{T-\epsilon_{0}}\left[\|\widehat{\psi}\|_{H^{\frac{1}{2}+\epsilon}(\Omega)}^{2}+\left\|\frac{\partial^{2} \widehat{\phi}}{\partial \tau^{2}}\right\|_{L^{2}\left(\Gamma_{1}\right)}^{2}+\left\|\frac{\partial^{2} \widehat{\phi}}{\partial \nu^{2}}\right\|_{L^{2}\left(\Gamma_{1}\right)}^{2}+2\left\|\frac{\partial^{2} \widehat{\phi}}{\partial \tau \partial \nu}\right\|_{L^{2}\left(\Gamma_{1}\right)}^{2}\right. \\
& \left.+\left\|\widehat{\phi}_{x}\right\|_{H^{\frac{1}{2}+\epsilon}(\Omega)}^{2}+\left\|\widehat{\phi}_{y}\right\|_{H^{\frac{1}{2}+\epsilon}(\Omega)}^{2}\right] d t \\
& \leq C_{T}\left(\int_{0}^{T}\left[\left\|c_{0} \widehat{\psi}+c_{1} \widehat{\psi}_{t}+c_{2} \widehat{\phi}+c_{3} \widehat{\phi}_{t}+c_{4} \Delta \widehat{\phi}\right\|_{\left[H^{\frac{3}{2}-\epsilon}(\Omega)\right.}^{2}\right]^{\prime}+\|\widehat{\psi}\|_{H^{\frac{1}{2}+\epsilon}(\Omega)}^{2}\right. \\
& \left.+\left\|\gamma \frac{\partial}{\partial \nu}\left(\xi^{2} \widehat{\phi}+2 \xi \widehat{\phi}_{t}\right)\right\|_{L^{2}\left(\Gamma_{1}\right)}^{2}\right] d t \\
& \left.+\int_{0}^{T}\left[\left\|\nabla \widehat{\phi}_{t}\right\|_{L^{2}\left(\Gamma_{1}\right)}^{2}+\|\hat{\phi}\|_{H^{\frac{3}{2}+\epsilon}(\Omega)}^{2}+\left\|\widehat{\phi}_{t}\right\|_{H^{\frac{1}{2}+\epsilon}(\Omega)}^{2}\right] d t\right) \\
& \left.\leq C_{T} \int_{0}^{T}\left[\left\|\nabla \widehat{\phi}_{t}\right\|_{L^{2}\left(\Gamma_{1}\right)}^{2}+\left\|c_{1} \widehat{\psi}_{t}+c_{4} \Delta \widehat{\phi}\right\|_{\left[H^{\frac{3}{2}-\epsilon}(\Omega)\right.}^{2}\right]^{\prime}+\right] d t+\text { I.o.t. }\left(\widehat{\phi}, \widehat{\phi}_{t}, \widehat{\psi}\right) \text {. }
\end{aligned}
$$

To handle the term $\int_{0}^{T}\left\|c_{1} \widehat{\psi}_{t}+c_{4} \Delta \widehat{\phi}\right\|_{\left[H^{\frac{3}{2}-\epsilon}(\Omega)\right]^{\prime}}^{2} d t$, we use Proposition 4.4 in the appendix below and the fact that $\widehat{\psi}_{t}=-\xi \widehat{\psi}(t)+e^{-\xi t} \psi_{t}(t)$ and $\widehat{\phi}(t)=e^{-\xi t} \phi(t)$ to have

$$
\begin{aligned}
& \left.\int_{0}^{T}\left\|c_{1} \widehat{\psi}_{t}+c_{4} \Delta \widehat{\phi}\right\|_{\left[H^{\frac{3}{2}-\epsilon}(\Omega)\right.}^{2}\right]^{\prime} d t \\
= & \left.\int_{0}^{T}\left\|-\xi c_{1} \widehat{\psi}(t)+c_{1} e^{-\xi t} \psi_{t}(t)+c_{4} e^{-\xi t} \Delta \phi(t)\right\|_{\left[H^{\frac{3}{2}-\epsilon}(\Omega)\right.}^{2}\right]^{\prime} d t \\
\leq & C \int_{0}^{T}\left[\|\widehat{\psi}\|_{L^{2}(\Omega)}^{2}+\|\phi\|_{H^{\frac{3}{2}+\epsilon}(\Omega)}^{2}+\|\psi\|_{H^{\frac{1}{2}+\epsilon}(\Omega)}^{2}+\left\|\phi_{t}\right\|_{H^{\frac{1}{2}+\epsilon}(\Omega)}^{2}+\left\|\frac{\partial \phi_{t}}{\partial \nu}\right\|_{L^{2}\left(\Gamma_{1}\right)}^{2}\right] d t \\
\leq & C_{T} \int_{0}^{T}\left\|\nabla \widehat{\phi}_{t}\right\|_{L^{2}\left(\Gamma_{1}\right)}^{2} d t+\text { l.o.t. }\left(\widehat{\phi}, \widehat{\phi}_{t}, \widehat{\psi}\right) .
\end{aligned}
$$


Collectively, estimates (2.52) and (2.53) then give

$$
\begin{aligned}
& -\int_{\epsilon_{0}}^{T-\epsilon_{0}} \alpha\left(\widehat{\psi}, \frac{\partial}{\partial \nu}(\bar{h} \cdot \nabla \widehat{\phi})\right)_{L^{2}\left(\Gamma_{1}\right)} d t \\
& \quad \leq C_{T} \int_{0}^{T}\left\|\nabla \widehat{\phi}_{t}\right\|_{L^{2}\left(\Gamma_{1}\right)}^{2} d t+\text { l.o.t. }\left(\widehat{\phi}, \widehat{\phi}_{t}, \widehat{\psi}\right) .
\end{aligned}
$$

(A.6) In the same way as in (A.5) we have

$$
\begin{gathered}
-\int_{\epsilon_{0}}^{T-\epsilon_{0}} \int_{\Gamma_{1}} \frac{\bar{h} \cdot \nu}{2}\left[\left(\frac{\partial^{2} \widehat{\phi}}{\partial x^{2}}\right)^{2}+\left(\frac{\partial^{2} \widehat{\phi}}{\partial y^{2}}\right)^{2}+2 \mu\left(\frac{\partial^{2} \widehat{\phi}}{\partial x^{2}}\right)\left(\frac{\partial^{2} \widehat{\phi}}{\partial y^{2}}\right)\right. \\
\left.+2(1-\mu)\left(\frac{\partial^{2} \widehat{\phi}}{\partial x \partial y}\right)^{2}\right] d t d \Gamma \\
\leq C_{T} \int_{0}^{T}\left\|\nabla \widehat{\phi}_{t}\right\|_{L^{2}\left(\Gamma_{1}\right)}^{2}+\text { l.o.t. }\left(\widehat{\phi}, \widehat{\phi}_{t}, \widehat{\psi}\right) .
\end{gathered}
$$

(A.7) Finally,

$$
\frac{1}{2} \int_{\epsilon_{0}}^{T-\epsilon_{0}} \int_{\Gamma_{1}} \bar{h} \cdot \nu\left(\widehat{\phi}_{t}^{2}+\gamma\left|\nabla \widehat{\phi}_{t}\right|^{2}\right) d \Gamma d t \leq C \int_{0}^{T}\left\|\nabla \widehat{\phi}_{t}\right\|_{L^{2}\left(\Gamma_{1}\right)}^{2} d t+\text { I.o.t. }\left(\widehat{\phi}, \widehat{\phi}_{t}, \widehat{\psi}\right) .
$$

Estimate (2.39) now comes about by stringing together (2.36), (2.40)-(2.42), (2.50), and (2.56), and taking $\epsilon>0$ small enough.

Lemma 2.6. For $T>T_{0} \equiv 2 \sqrt{\frac{2 \gamma}{1-\mu}} \max _{(x, y) \in \bar{\Omega}}|\bar{h}(x, y)|$, the solution $\left[\widehat{\phi}, \widehat{\phi}_{t}, \widehat{\psi}\right]$ of (2.15) satisfies the following estimate:

$$
\begin{array}{r}
\int_{0}^{T} E_{\widehat{\phi}}(t) d t+E_{\widehat{\phi}}(T)+\int_{0}^{T}\left\|A_{R}^{\frac{1}{2}} \widehat{\psi}\right\|_{L^{2}(\Omega)}^{2} d t \\
\leq C_{T}\left(\left\|\psi_{0}\right\|_{L^{2}(\Omega)}^{2}+\int_{0}^{T}\left\|\nabla \widehat{\phi}_{t}\right\|_{L^{2}\left(\Gamma_{1}\right)}^{2} d t\right)+\text { I.o.t. }\left(\widehat{\phi}, \widehat{\phi}_{t}, \widehat{\psi}\right) .
\end{array}
$$

Proof. We have for any $\epsilon_{0} \in(0, T)$,

$$
\begin{aligned}
& \int_{0}^{T} E_{\widehat{\phi}}(t) d t=\int_{0}^{\epsilon_{0}} E_{\widehat{\phi}}(t) d t+\int_{T-\epsilon_{0}}^{T} E_{\widehat{\phi}}(t) d t+\int_{\epsilon_{0}}^{T-\epsilon_{0}} E_{\widehat{\phi}}(t) d t \\
\leq & \frac{2 \epsilon_{0}(1+\epsilon)}{1-\epsilon} E_{\widehat{\phi}}(T)+\frac{2 \epsilon_{0} \epsilon}{1-\epsilon} \int_{0}^{T}\left[E_{\widehat{\phi}}(t)+\left\|A_{R}^{\frac{1}{2}} \widehat{\psi}(t)\right\|_{L^{2}(\Omega)}^{2}\right] d t+\int_{\epsilon_{0}}^{T-\epsilon_{0}} E_{\widehat{\phi}}(t) d t \\
& +C_{\epsilon} \int_{0}^{T}\left\|\nabla \widehat{\phi}_{t}\right\|_{L^{2}\left(\Gamma_{1}\right)}^{2} d t+\text { I.o.t. }\left(\widehat{\phi}, \widehat{\phi}_{t}, \widehat{\psi}\right) \\
& \text { (after applying Lemma 2.2(b) twice) } \\
\leq & C^{*}\left(E_{\widehat{\phi}}\left(T-\epsilon_{0}\right)+E_{\widehat{\phi}}\left(\epsilon_{0}\right)\right)+\frac{2 \epsilon_{0}(1+\epsilon)}{1-\epsilon} E_{\widehat{\phi}}(T)+\frac{2 \epsilon_{0} \epsilon}{1-\epsilon} \int_{0}^{T} E_{\widehat{\phi}}(t) d t \\
(2.58)+ & \frac{2 \epsilon\left(1+\epsilon_{0}\right)}{1-\epsilon} \int_{0}^{T}\left\|A_{R}^{\frac{1}{2}} \widehat{\psi}\right\|_{L^{2}(\Omega)}^{2} d t+C_{T, \epsilon} \int_{0}^{T}\left\|\nabla \widehat{\phi}_{t}\right\|_{L^{2}\left(\Gamma_{1}\right)}^{2} d t+\text { I.o.t. }\left(\widehat{\phi}, \widehat{\phi}_{t}, \widehat{\psi}\right),
\end{aligned}
$$


after applying Lemma 2.5 with $C^{*} \geq \sqrt{\frac{2 \gamma}{1-\mu}} \max _{[x, y] \in \bar{\Omega}}|\bar{h}(x, y)|$, and $\tilde{\epsilon} \equiv \frac{2 \epsilon}{1-\epsilon}$ therein. Applying Lemma 2.2(b) twice more to the right-hand side of (2.58) yields now

$$
\begin{aligned}
& \int_{0}^{T} E_{\widehat{\phi}}(t) d t \leq 2\left(\epsilon_{0}+C^{*}\right) \frac{1+\epsilon}{1-\epsilon} E_{\widehat{\phi}}(T)+\frac{2 \epsilon\left(\epsilon_{0}+C^{*}\right)}{1-\epsilon} \int_{0}^{T} E_{\widehat{\phi}}(t) d t \\
& \quad+\frac{2 \epsilon\left(1+\epsilon_{0}+C^{*}\right)}{1-\epsilon} \int_{0}^{T}\left\|A_{R}^{\frac{1}{2}} \widehat{\psi}\right\|_{L^{2}(\Omega)}^{2} d t+C_{T, \epsilon} \int_{0}^{T}\left\|\nabla \widehat{\phi}_{t}\right\|_{L^{2}\left(\Gamma_{1}\right)}^{2} d t \\
& \quad+\text { I.o.t. }(\widehat{\phi}, \widehat{\phi}, \widehat{\psi}) .
\end{aligned}
$$

Moreover, we have by (2.16)

$$
\int_{0}^{T} E_{\widehat{\phi}}(t) d t=T E_{\widehat{\phi}}(T)+\int_{0}^{T} \mathcal{F}(T, t) d t
$$

where the function $\mathcal{F}$ is as defined in (2.34). Combining (2.59) and (2.60) yields

$$
\begin{aligned}
& T E_{\widehat{\phi}}(T)+\int_{0}^{T} \mathcal{F}(T, t) d t \leq 2\left(\epsilon_{0}+C^{*}\right) \frac{1+\epsilon}{1-\epsilon} E_{\widehat{\phi}}(T)+\frac{2 \epsilon\left(\epsilon_{0}+C^{*}\right)}{1-\epsilon} \int_{0}^{T} E_{\widehat{\phi}}(t) d t \\
& \quad+\frac{2 \epsilon\left(1+\epsilon_{0}+C^{*}\right)}{1-\epsilon} \int_{0}^{T}\left\|A_{R}^{\frac{1}{2}} \widehat{\psi}\right\|_{L^{2}(\Omega)}^{2} d t+C_{T, \epsilon} \int_{0}^{T}\left\|\nabla \widehat{\phi}_{t}\right\|_{L^{2}\left(\Gamma_{1}\right)}^{2} d t+\text { I.o.t. }\left(\widehat{\phi}, \widehat{\phi}_{t}, \widehat{\psi}\right) .
\end{aligned}
$$

To use this inequality, we integrate both sides of (2.17) (with $s=T$ therein) so as to have

$$
\begin{aligned}
\int_{0}^{T} \mathcal{F}(T, t) d t \leq & \epsilon(T+1) \int_{0}^{T} E_{\widehat{\phi}}(t) d t+\epsilon T E_{\widehat{\phi}}(T)+\epsilon T \int_{0}^{T}\left\|A_{R}^{\frac{1}{2}} \widehat{\psi}\right\|_{L^{2}(\Omega)}^{2} d t \\
& +C_{T, \epsilon} \int_{0}^{T}\left\|\nabla \widehat{\phi}_{t}\right\|_{L^{2}\left(\Gamma_{1}\right)}^{2} d t+\text { I.o.t. }\left(\widehat{\phi}, \widehat{\phi}_{t}, \widehat{\psi}\right) .
\end{aligned}
$$

Combining (2.61) and (2.62), we thus obtain

$$
\begin{aligned}
T E_{\widehat{\phi}}(T) \leq & {\left[\frac{2\left(\epsilon_{0}+C^{*}\right)(1+\epsilon)}{1-\epsilon}+\epsilon T\right] E_{\widehat{\phi}}(T) } \\
& +\epsilon\left[\frac{2\left(\epsilon_{0}+C^{*}\right)}{1-\epsilon}+(T+1)\right] \int_{0}^{T} E_{\widehat{\phi}}(t) d t \\
& +\epsilon\left[\frac{2\left(1+\epsilon_{0}+C^{*}\right)}{1-\epsilon}+T\right] \int_{0}^{T}\left\|A_{R}^{\frac{1}{2}} \widehat{\psi}\right\|_{L^{2}(\Omega)}^{2} d t+C_{T, \epsilon} \int_{0}^{T}\left\|\nabla \widehat{\phi}_{t}\right\|_{L^{2}\left(\Gamma_{1}\right)}^{2} d t \\
& + \text { l.o.t. }\left(\widehat{\phi}, \widehat{\phi}_{t}, \widehat{\psi}\right) .
\end{aligned}
$$

Taking now $T>\frac{2\left(\epsilon_{0}+C^{*}\right)(1+\epsilon)}{(1-\epsilon)^{2}}$, or what is the same, $T>2 C^{*}$ for $\epsilon$ and $\epsilon_{0}$ small enough, we then have

$$
\begin{aligned}
E_{\widehat{\phi}}(T) \leq & C_{T, \epsilon} \int_{0}^{T}\left\|\nabla \widehat{\phi}_{t}\right\|_{L^{2}\left(\Gamma_{1}\right)}^{2} d t+\epsilon \widetilde{C}_{T}\left[\int_{0}^{T} E_{\widehat{\phi}}(t) d t+\int_{0}^{T}\left\|A_{R}^{\frac{1}{2}} \widehat{\psi}\right\|_{L^{2}(\Omega)}^{2} d t\right] \\
& + \text { I.o.t. }\left(\widehat{\phi}, \widehat{\phi}_{t}, \widehat{\psi}\right),
\end{aligned}
$$


where throughout $\widetilde{C}_{T}$ will denote a constant independent of $\epsilon$ and $\epsilon_{0}$ (small enough).

In turn, applying this to (2.59), we have

$$
\begin{gathered}
\int_{0}^{T} E_{\widehat{\phi}}(t) d t \leq C_{T, \epsilon} \int_{0}^{T}\left\|\nabla \widehat{\phi}_{t}\right\|_{L^{2}\left(\Gamma_{1}\right)}^{2} d t+\frac{2 \epsilon\left(\epsilon_{0}+C^{*}\right)\left[(1+\epsilon) \widetilde{C}_{T}+1\right]}{1-\epsilon} \int_{0}^{T} E_{\widehat{\phi}}(t) d t \\
+\frac{2 \epsilon\left[\left(\epsilon_{0}+C^{*}\right)(1+\epsilon) \widetilde{C}_{T}+\left(1+\epsilon_{0}+C^{*}\right)\right]}{1-\epsilon} \int_{0}^{T}\left\|A_{R}^{\frac{1}{2}} \widehat{\psi}\right\|_{L^{2}(\Omega)}^{2} d t+\text { I.o.t. }\left(\widehat{\phi}, \widehat{\phi}_{t}, \widehat{\psi}\right)
\end{gathered}
$$

from which follows the estimate, for $\epsilon, \epsilon_{0}>0$ small enough,

$$
\int_{0}^{T} E_{\widehat{\phi}}(t) d t \leq C_{T, \epsilon} \int_{0}^{T}\left\|\nabla \widehat{\phi}_{t}\right\|_{L^{2}\left(\Gamma_{1}\right)}^{2} d t+\epsilon \widetilde{C}_{T} \int_{0}^{T}\left\|A_{R}^{\frac{1}{2}} \widehat{\psi}\right\|_{L^{2}(\Omega)}^{2} d t+\text { I.o.t. }\left(\widehat{\phi}, \widehat{\phi}_{t}, \widehat{\psi}\right)
$$

Coupling together (2.64) and (2.65), we have the following preliminary inequality for the mechanical energy, again for $T>2 C^{*}$ :

$$
\begin{aligned}
& \int_{0}^{T} E_{\widehat{\phi}}(t) d t+E_{\widehat{\phi}}(T) \\
& \quad \leq C_{T, \epsilon} \int_{0}^{T}\left\|\nabla \widehat{\phi}_{t}\right\|_{L^{2}\left(\Gamma_{1}\right)}^{2} d t+\epsilon \widetilde{C}_{T} \int_{0}^{T}\left\|A_{R}^{\frac{1}{2}} \widehat{\psi}\right\|_{L^{2}(\Omega)}^{2} d t+\text { I.o.t. }\left(\widehat{\phi}, \widehat{\phi}_{t}, \widehat{\psi}\right) .
\end{aligned}
$$

It remains to estimate the thermal component. To this end, we can multiply (2.11) by $\widehat{\psi}$, integrate in time and space, use the characterization (1.15) and (2.8) to have

$$
\begin{aligned}
& \eta \int_{0}^{T}\left\|A_{R}^{\frac{1}{2}} \widehat{\psi}\right\|_{L^{2}(\Omega)}^{2} d t=\left[\frac{\beta}{2}\left\|e^{-\xi t} \psi(t)\right\|_{L^{2}(\Omega)}^{2}\right]_{t=0}^{t=T}+\xi \int_{0}^{T}(\beta \widehat{\psi}-\alpha \Delta \widehat{\phi}, \widehat{\psi})_{L^{2}(\Omega)} d t \\
& \quad+\alpha \int_{0}^{T}\left[\left(\nabla \widehat{\phi}_{t}, \nabla \widehat{\psi}\right)_{L^{2}(\Omega)}-\left(\frac{\partial \widehat{\phi}_{t}}{\partial \nu}, \widehat{\psi}\right)_{L^{2}\left(\Gamma_{1}\right)}\right] d t .
\end{aligned}
$$

Majorizing this expression results in

$$
\begin{aligned}
& \eta \int_{0}^{T}\left\|A_{R}^{\frac{1}{2}} \widehat{\psi}\right\|_{L^{2}(\Omega)}^{2} d t \leq C_{\widetilde{\epsilon}}\left(\left\|\psi_{0}\right\|_{L^{2}(\Omega)}^{2}+\int_{0}^{T}\|\nabla \widehat{\phi} t\|_{L^{2}\left(\Gamma_{1}\right)}^{2} d t+\int_{0}^{T} E_{\widehat{\phi}}(t) d t\right) \\
& +\widetilde{\epsilon} \int_{0}^{T}\left\|A_{R}^{\frac{1}{2}} \widehat{\psi}\right\|_{L^{2}(\Omega)}^{2} d t+\text { l.o.t. }\left(\widehat{\phi}, \widehat{\phi}_{t}, \widehat{\psi}\right),
\end{aligned}
$$

and taking $\tilde{\epsilon}>0$ small enough above, this becomes

$$
\int_{0}^{T}\left\|A_{R}^{\frac{1}{2}} \widehat{\psi}\right\|_{L^{2}(\Omega)}^{2} d t \leq C_{1}\left(\left\|\psi_{0}\right\|_{L^{2}(\Omega)}^{2}+\int_{0}^{T}\left\|\nabla \widehat{\phi}_{t}\right\|_{L^{2}\left(\Gamma_{1}\right)}^{2} d t+\int_{0}^{T} E_{\widehat{\phi}}(t) d t\right)
$$

$(2.68)+$ I.o.t. $\left(\widehat{\phi}, \widehat{\phi}_{t}, \widehat{\psi}\right)$,

where $C_{1}=\frac{C_{\tilde{\epsilon}}}{\eta-\tilde{\epsilon}}$.

Combining (2.66) and (2.68), we have 


$$
\begin{aligned}
& \int_{0}^{T} E_{\widehat{\phi}}(t) d t+E_{\widehat{\phi}}(T) \\
& \quad \leq C_{T, \epsilon} \int_{0}^{T}\left\|\nabla \widehat{\phi}_{t}\right\|_{L^{2}\left(\Gamma_{1}\right)}^{2} d t+\epsilon \widetilde{C}_{T} C_{1}\left(\left\|\psi_{0}\right\|_{L^{2}(\Omega)}^{2}+\int_{0}^{T} E_{\widehat{\phi}}(t) d t\right)+\text { I.o.t. }\left(\widehat{\phi}, \widehat{\phi}_{t}, \widehat{\psi}\right),
\end{aligned}
$$

from which we obtain for $\epsilon>0$ small enough

$$
\int_{0}^{T} E_{\widehat{\phi}}(t) d t+E_{\widehat{\phi}}(T) \leq C_{T, \epsilon}\left(\left\|\psi_{0}\right\|_{L^{2}(\Omega)}^{2}+\int_{0}^{T}\left\|\nabla \widehat{\phi}_{t}\right\|_{L^{2}\left(\Gamma_{1}\right)}^{2} d t\right)+\text { I.o.t. }\left(\widehat{\phi}, \widehat{\phi}_{t}, \widehat{\psi}\right) \text {. }
$$

The final estimate (2.57) finally comes about by combining (2.70) and (2.68).

Conclusion of the proof of Theorem 2.1. Assume initially that $\left[\phi_{0}, \phi_{1}, \psi_{0}\right] \in$ $D\left(\mathcal{A}_{\gamma}^{*}\right)$. Through the change of variable $\widehat{\phi}(t)=e^{-\xi t} \phi(t)$ and $\widehat{\psi}(t)=e^{-\xi t} \psi(t)$, where again $\left[\widehat{\phi}, \widehat{\phi}_{t}, \widehat{\psi}\right]$ solves $(2.15)$ and $\xi \equiv \frac{\alpha^{2}}{2 \gamma \eta}>0$, we have for $T>T_{0} \equiv$ $2 \sqrt{\frac{2 \gamma}{1-\mu}} \max _{[x, y] \in \bar{\Omega}}|\bar{h}(x, y)|$

$$
\begin{aligned}
& \int_{0}^{T} E_{\phi}(t) d t+\int_{0}^{T}\left\|A_{R}^{\frac{1}{2}} \psi(t)\right\|_{L^{2}(\Omega)}^{2} d t+E_{\phi}(T) \\
= & \int_{0}^{T}\left[\left\|\AA^{\frac{1}{2}} e^{\xi t} \widehat{\phi}(t)\right\|_{L^{2}(\Omega)}^{2}+\left\|P_{\gamma}^{\frac{1}{2}}\left(e^{\xi t} \widehat{\phi}_{t}(t)+\xi e^{\xi t} \widehat{\phi}(t)\right)\right\|_{L^{2}(\Omega)}^{2}+\left\|A_{R}^{\frac{1}{2}} e^{\xi t} \widehat{\psi}(t)\right\|_{L^{2}(\Omega)}^{2}\right] d t \\
& +\left\|\AA^{\frac{1}{2}} e^{\xi T} \widehat{\phi}(T)\right\|_{L^{2}(\Omega)}^{2}+\left\|P_{\gamma}^{\frac{1}{2}}\left(e^{\xi T} \widehat{\phi}_{t}(T)+\xi e^{T} \widehat{\phi}(T)\right)\right\|_{L^{2}(\Omega)}^{2} \\
\leq & C_{T}\left(\left\|\psi_{0}\right\|_{L^{2}(\Omega)}^{2}+\int_{0}^{T}\left\|\nabla \widehat{\phi}_{t}\right\|_{L^{2}\left(\Gamma_{1}\right)}^{2} d t\right)+\text { l.o.t. }\left(\widehat{\phi}, \widehat{\phi}_{t}, \widehat{\psi}\right)
\end{aligned}
$$

(after using estimate (2.57))

$$
\begin{aligned}
\leq & C_{T}\left(\left\|\psi_{0}\right\|_{L^{2}(\Omega)}^{2}+\int_{0}^{T}\left\|\nabla\left(e^{-\xi t} \phi_{t}(t)-\xi e^{-\xi t} \phi(t)\right)\right\|_{L^{2}\left(\Gamma_{1}\right)}^{2} d t\right) \\
& + \text { I.o.t. }\left(e^{-\xi t} \phi, e^{-\xi t} \phi_{t}-\xi e^{-\xi t} \phi, e^{-\xi t} \psi\right) \\
\leq & C_{T}\left(\left\|\psi_{0}\right\|_{L^{2}(\Omega)}^{2}+\int_{0}^{T}\left\|\nabla \phi_{t}(t)\right\|_{L^{2}\left(\Gamma_{1}\right)}^{2} d t\right)+\text { I.o.t. }\left(\phi, \phi_{t}, \psi\right) .
\end{aligned}
$$

This gives the desired inequality (2.5).

2.3. Conclusion of the proof of Theorem 1.2. For $\left[\phi_{0}, \phi_{1}\right] \in D\left(\mathcal{L}_{T}^{*} \Pi^{*}\right)$, we immediately have from Theorem 2.1 the following corollary.

Corollary 2.7. For $\left[\phi_{0}, \phi_{1}\right] \in D\left(\mathcal{L}_{T}^{*} \Pi^{*}\right)$ and $T>T_{0} \equiv 2 \sqrt{\frac{2 \gamma}{1-\mu}} \max _{[x, y] \in \bar{\Omega}}|\bar{h}(x, y)|$, the corresponding solution $\left[\phi, \phi_{t}, \psi\right]$ of (1.60) satisfies the following inequality:

$$
\int_{0}^{T} E_{\phi}(t) d t+E_{\phi}(T)+\int_{0}^{T}\left\|A_{R}^{\frac{1}{2}} \psi\right\|_{L^{2}(\Omega)}^{2} d t \leq C_{T} \int_{0}^{T}\left\|\nabla \phi_{t}\right\|_{L^{2}\left(\Gamma_{1}\right)}^{2} d t+\text { I.o.t. }\left(\phi, \phi_{t}, \psi\right) \text {. }
$$


We will have the desired inequality (2.2) upon the elimination of the tainting lower order terms in (2.72). To this end, we invoke a (by now) classical compactnessuniqueness argument (see, e.g., [13] and [2]), which makes crucial use of the new Holmgren-type uniqueness result for the thermoelastic system recently derived by Isakov in [10]. It is at this point that the boundary trace $\left.\psi\right|_{\Gamma_{2}}$, corresponding to the control $u_{3}$, comes into play.

Lemma 2.8. Let $T^{*}$ be as defined in (1.6). Then for $T>T^{*}$ and initial data $\left[\phi_{0}, \phi_{1}\right] \in D\left(\mathcal{L}_{T}^{*} \Pi^{*}\right)$, there exists a $C_{T}$ such that the following estimate holds true for the solution of (1.60):

$$
\begin{aligned}
\|\phi\|_{L^{\infty}\left(0, T ; H^{\frac{3}{2}+\epsilon}(\Omega)\right)}^{2} & +\left\|\phi_{t}\right\|_{L^{\infty}\left(0, T ; H^{\frac{1}{2}+\epsilon}(\Omega)\right)}^{2}+\|\psi\|_{L^{\infty}\left(0, T ; H^{-\frac{1}{2}+\epsilon}(\Omega)\right)}^{2}+\int_{0}^{T}\|\psi\|_{H^{\frac{1}{2}+\epsilon}(\Omega)}^{2} d t \\
& \leq C_{T}\left(\int_{0}^{T}\left\|\nabla \phi_{t}\right\|_{L^{2}\left(\Gamma_{1}\right)}^{2} d t+\|\psi\|_{\left[H^{s}\left((0, T) \times \Gamma_{2}\right)\right]^{\prime}}^{2}\right) .
\end{aligned}
$$

Proof. If the proposition is false, then there exists a sequence $\left\{\left[\phi_{0}^{(n)}, \phi_{1}^{(n)}\right]\right\}_{n=1}^{\infty} \subseteq$ $D\left(\mathcal{L}_{T}^{*} \Pi^{*}\right)$, and a corresponding solution sequence $\left\{\left[\phi^{(n)}, \phi_{t}^{(n)}, \psi^{(n)}\right]\right\}_{n=1}^{\infty}$ to $(1.60)$, which satisfies

$$
\begin{gathered}
\left.\left\|\phi^{(n)}\right\|_{L^{\infty}\left(0, T ; H^{\frac{3}{2}+\epsilon}(\Omega)\right.}^{2}+\left\|\phi_{t}^{(n)}\right\|_{L^{\infty}\left(0, T ; H^{\frac{1}{2}+\epsilon}(\Omega)\right)}^{2}+\left\|\psi^{(n)}\right\|_{L^{\infty}\left(0, T ; H^{-\frac{1}{2}+\epsilon}(\Omega)\right.}^{2}\right) \\
\quad+\int_{0}^{T}\left\|\psi^{(n)}\right\|_{H^{\frac{1}{2}+\epsilon}(\Omega)}^{2} d t=1 \quad \forall n, \\
(2.74) \quad \lim _{n \rightarrow \infty} \int_{0}^{T}\left\|\nabla \phi_{t}^{(n)}\right\|_{L^{2}\left(\Gamma_{1}\right)}^{2} d t+\left\|\psi^{(n)}\right\|_{\left[H^{s}\left((0, T) \times \Gamma_{2}\right)\right]^{\prime}}^{2}=0 .
\end{gathered}
$$

As $T>2 \sqrt{\frac{2 \gamma}{1-\mu}} \max _{[x, y] \in \bar{\Omega}}|\bar{h}(x, y)|$, we have the existence of the inequality (2.72). This and (2.74)-(2.75) then imply the boundedness of the sequence

$$
\begin{aligned}
& \left\{\int_{0}^{T}\left[\left\|\left[\begin{array}{l}
\phi^{(n)}(t) \\
\phi_{t}^{(n)}(t)
\end{array}\right]\right\|_{D\left(\AA^{\frac{1}{2}}\right) \times H_{\Gamma_{0}, \gamma}^{1}(\Omega)}^{2}+\left\|A_{R}^{\frac{1}{2}} \psi^{(n)}(t)\right\|_{L^{2}(\Omega)}^{2}\right] d t\right. \\
& \left.+\left\|\left[\begin{array}{l}
\phi_{0}^{(n)} \\
\phi_{1}^{(n)}
\end{array}\right]\right\|_{D\left(\AA^{\frac{1}{2}}\right) \times H_{\Gamma_{0}, \gamma}^{1}(\Omega)}^{2}\right\}_{n=1}^{\infty} .
\end{aligned}
$$

There thus exists a subsequence, still denoted here as $\left\{\left[\phi_{0}^{(n)}, \phi_{1}^{(n)}\right]\right\}_{n=1}^{\infty}$, and $\left[\widetilde{\phi}_{0}, \widetilde{\phi}_{1}\right] \in$ $D\left(\AA^{\frac{1}{2}}\right) \times H_{\Gamma_{0}, \gamma}^{1}(\Omega)$, such that

$$
\begin{aligned}
& \phi_{0}^{(n)} \rightarrow \widetilde{\phi}_{0} \text { in } D\left(\AA^{\frac{1}{2}}\right) \text { weakly, } \\
& \phi_{1}^{(n)} \rightarrow \widetilde{\phi}_{1} \text { in } H_{\Gamma_{0}, \gamma}^{1}(\Omega) \text { weakly. }
\end{aligned}
$$

If we further denote $\left[\widetilde{\phi}, \widetilde{\phi}_{t}, \widetilde{\psi}\right]$ as the solution to (1.60), corresponding to initial data $\left[\widetilde{\phi}_{0}, \widetilde{\phi}_{1}, 0\right]$, then a fortiori,

$$
\left[\phi^{(n)}, \phi_{t}^{(n)}, \psi^{(n)}\right] \rightarrow\left[\widetilde{\phi}, \widetilde{\phi}_{t}, \widetilde{\psi}\right] \text { in } L^{\infty}\left(0, T ; \mathbf{H}_{\gamma}\right) \text { weak star. }
$$


From Proposition 4.3 of the appendix, we have that $\left\{\phi_{t t}^{(n)}\right\}_{n=1}^{\infty}$ is bounded in $L^{\infty}\left(0, T ;\left[D\left(\AA^{\frac{1}{2}} P_{\gamma}^{-1}\right)\right]^{\prime}\right)$, inasmuch as $\left\{\left\|\left[\phi_{0}^{(n)}, \phi_{1}^{(n)}\right]\right\|_{D\left(\AA^{\frac{1}{2}}\right) \times H_{\Gamma_{0}, \gamma}^{1}(\Omega)}\right\}_{n=1}^{\infty}$ is bounded in $D\left(\AA^{\frac{1}{2}}\right) \times H_{\Gamma_{0}, \gamma}^{1}(\Omega)$. Also, from Proposition 4.4 we have that $\psi_{t}^{(n)} \in L^{2}\left(0, T ;\left[H^{\frac{3}{2}-\epsilon}(\Omega)\right]^{\prime}\right)$ for all $n$, with the estimate

$$
\int_{0}^{T}\left\|\psi_{t}^{(n)}\right\|_{\left[H^{\frac{3}{2}-\epsilon}(\Omega)\right]^{\prime}}^{2} d t \leq C \int_{0}^{T}\left\|\nabla \phi_{t}^{(n)}\right\|_{L^{2}\left(\Gamma_{1}\right)}^{2} d t+\text { I.o.t. }\left(\phi, \phi_{t}, \psi\right)
$$

and this combined with $(2.74)-(2.75)$ yields that $\left\{\psi_{t}^{(n)}\right\}_{n=1}^{\infty}$ is bounded in $L^{2}\left(0, T ;\left[H^{\frac{3}{2}-\epsilon}(\Omega)\right]^{\prime}\right)$. This boundedness of $\left\{\left[\phi_{t t}^{(n)}, \psi_{t}^{(n)}\right]\right\}_{n=1}^{\infty}$, and that for the sequence posted in (2.76), allows us to deduce through a compactness result of Simon's in $[24]$ that

$$
\begin{aligned}
& \phi^{(n)} \rightarrow \widetilde{\phi} \text { strongly in } L^{\infty}\left(0, T ; H^{\frac{3}{2}+\epsilon}(\Omega)\right), \\
& \phi_{t}^{(n)} \rightarrow \widetilde{\phi}_{t} \text { strongly in } L^{\infty}\left(0, T ; H^{\frac{1}{2}+\epsilon}(\Omega)\right), \\
& \psi^{(n)} \rightarrow \widetilde{\psi} \text { strongly in } L^{2}\left(0, T ; H^{\frac{1}{2}+\epsilon}(\Omega)\right), \\
& \psi^{(n)} \rightarrow \widetilde{\psi} \text { strongly in } L^{\infty}\left(0, T ; H^{-\frac{1}{2}+\epsilon}(\Omega)\right) .
\end{aligned}
$$

These convergences and (2.74) thus give

$$
\begin{aligned}
& \|\widetilde{\phi}\|_{L^{\infty}\left(0, T ; H^{\frac{3}{2}+\epsilon}(\Omega)\right)}^{2}+\left\|\widetilde{\phi}_{t}\right\|_{L^{\infty}\left(0, T ; H^{\frac{1}{2}+\epsilon}(\Omega)\right)}^{2}+\|\widetilde{\psi}\|_{L^{\infty}\left(0, T ; H^{-\frac{1}{2}+\epsilon}(\Omega)\right)}^{2} \\
& \quad+\int_{0}^{T}\|\widetilde{\psi}\|_{H^{\frac{1}{2}+\epsilon}(\Omega)}^{2} d t=1 .
\end{aligned}
$$

Moreover, the explicit representation of $\mathcal{L}_{T}^{*} \Pi^{*}$ in (1.59) and the convergences posted in (2.75) and $(2.77)-(2.79)$ give that $\left[\widetilde{\phi}_{0}, \widetilde{\phi}_{1}\right] \in D\left(\mathcal{L}_{T}^{*} \Pi^{*}\right)$, with

$$
\int_{0}^{T}\left\|\nabla \widetilde{\phi}_{t}\right\|_{H^{1}\left(\Gamma_{1}\right)}^{2} d t+\|\widetilde{\psi}\|_{\left[H^{s}\left((0, T) \times \Gamma_{2}\right)\right]^{\prime}}^{2}=0 .
$$

Now if we make the change of variable

$$
z=\widetilde{\phi}_{t}, \quad v=\widetilde{\psi}_{t},
$$

then using (2.82), $[z, v]$ solve the system

$$
\left\{\begin{array}{l}
\left\{\begin{array}{l}
z_{t t}-\gamma \Delta z_{t t}+\Delta^{2} z+\alpha \Delta v=0 \\
\beta v_{t}+\eta \Delta v-\sigma v-\alpha \Delta z_{t}=0
\end{array} \text { on }(0, \infty) \times \Omega,\right. \\
z=\frac{\partial z}{\partial \nu}=0 \text { on }(0, \infty) \times \Gamma, \\
\left\{\begin{array}{l}
\Delta z+(1-\mu) B_{1} z+\alpha v=0 \\
\frac{\partial \Delta z}{\partial \nu}+(1-\mu) \frac{\partial B_{2} z}{\partial \tau}-\gamma \frac{\partial z_{t t}}{\partial \nu}+\alpha \frac{\partial v}{\partial \nu}=0
\end{array} \text { on }(0, \infty) \times \Gamma_{1},\right. \\
\frac{\partial v}{\partial \nu}+\lambda v=0 \text { on }(0, \infty) \times \Gamma, \\
v=0 \text { on }(0, \infty) \times \Gamma_{2} .
\end{array}\right.
$$


Now by Isakov's theorem in [10, p. 3, Corollary 1.2], we have for

$$
T>2 \sqrt{\gamma} \cdot \sup _{[x, y] \in \Omega} d\left([x, y], \Gamma_{2}\right)
$$

that the uniqueness property for the thermoelastic system is obtained, so that the solution $[z, v]$ of $(2.83)$ is necessarily zero. Consequently $\widetilde{\phi}$ and $\widetilde{\psi}$ are each constants. From the essential boundary condition on $\Gamma_{0}$ in $(1.60)$, we then have $\widetilde{\phi}=0$ on $(0, T) \times$ $\Omega$. In turn, the free boundary conditions on $\Gamma_{1}$ give that $\widetilde{\psi}=0$ on $(0, T) \times \Omega$. Thus $[\widetilde{\phi}, \widetilde{\psi}]=[0,0]$, which contradicts the equality given in $(2.81)$. This concludes the proof of the lemma.

Corollary 2.7 and Lemma 2.8 in combination give inequality (2.2), the establishment of which verifies the surjectivity of the control to partial state map $\Pi \mathcal{L}_{T}$ : $D\left(\mathcal{L}_{T}\right) \subset \mathcal{U}_{s} \rightarrow D\left(\AA^{\frac{1}{2}}\right) \times H_{\Gamma_{0}, \gamma}^{1}(\Omega)$. This completes the proof of Theorem 1.2.

3. The proof of Theorem 1.1. Given the space $C^{r}\left(\Sigma_{2, T}\right)$, we consider system (1.1) under the influence of boundary controls in $\mathcal{U}_{r+1}$, as defined in (1.37). The controlled PDE is then approximately controllable in $\mathcal{U}_{r+1}$ for $T>2 \sqrt{\gamma} \cdot \sup _{[x, y] \in \Omega}$ $d\left([x, y], \Gamma_{2}\right)$. Indeed, if we take arbitrary $\left[\phi_{0}, \phi_{1}, \psi_{0}\right]$ from the null space of $\mathcal{L}_{T}^{*}$, then using the form of this operator given in (1.55), we have necessarily that $\left.\phi_{t}\right|_{\Gamma_{1}}=$ $\left.\frac{\partial \phi_{t}}{\partial \nu}\right|_{\Gamma_{1}}=0$, and $\left.\psi\right|_{\Gamma_{2}}=0$, where $\left[\phi, \phi_{t}, \psi\right]$ is the solution to (1.51). We can then use the uniqueness theorem of Isakov, in a fashion similar to that employed in Lemma 2.8, to show that $\left[\phi, \phi_{t}, \psi\right]=[0,0,0]$ on $(0, T) \times \Omega$ and, in particular, $\left[\phi_{0}, \phi_{1}, \psi_{0}\right]=[0,0,0]$.

A preliminary step (a regularity property of $\mathcal{L}_{T}$ ). With the designated control space $\mathcal{U}_{r+1}$ we then take $T>T^{*}$ so as to ensure both the approximate controllability of the entire system (1.1) and the exact controllability with respect to the displacement (see Theorem 1.2). In this event, we have the observability inequality $(2.2)$, and therewith one can show in a manner identical to that done in [14, Appendix B] that the operator

$$
\Pi \mathcal{L}_{T} \mathcal{L}_{T}^{*} \Pi^{*} \text { is an isomorphism from } D\left(\mathcal{L}_{T}^{*} \Pi^{*}\right) \text { into }\left[D\left(\mathcal{L}_{T}^{*} \Pi^{*}\right)\right]^{\prime},
$$

where the projection $\Pi$ onto $D\left(\AA^{\frac{1}{2}}\right) \times H_{\Gamma_{0}, \gamma}^{1}(\Omega)$ is as defined in (1.46). Consequently, we have

$$
\Pi \mathcal{L}_{T} \mathcal{L}_{T}^{*} \Pi^{*}\left(\Pi \mathcal{L}_{T} \mathcal{L}_{T}^{*} \Pi^{*}\right)^{-1} \Pi \in \mathcal{L}\left(\mathbf{H}_{\gamma}, D\left(\AA^{\frac{1}{2}}\right) \times H_{\Gamma_{0}, \gamma}^{1}(\Omega)\right) .
$$

Moreover, if we denote the maps $\mathcal{L}^{(1)}, \mathcal{L}^{(2)}$ by

$$
\begin{aligned}
\mathcal{L}^{(1)}\left[\begin{array}{l}
u_{1} \\
u_{2}
\end{array}\right](t) & =\int_{0}^{t} e^{\mathcal{A}_{\gamma}(t-s)} \mathcal{B}\left[\begin{array}{c}
u_{1}(s) \\
u_{2}(s) \\
0
\end{array}\right], \\
\mathcal{L}^{(2)} u(t) & =\int_{0}^{t} e^{\mathcal{A}_{\gamma}(t-s)} \mathcal{B}\left[\begin{array}{c}
0 \\
0 \\
u(s)
\end{array}\right]
\end{aligned}
$$

(cf. (1.42)), then by a standard energy method one can show that

$$
\mathcal{L}^{(2)}: L^{2}\left(0, T ; H^{-\frac{1}{2}}\left(\Gamma_{2}\right)\right) \rightarrow C\left([0, T] ; \mathbf{H}_{\gamma}\right) \text { continuously. }
$$


To handle $\mathcal{L}^{(1)}$ on the other hand, one must appeal to a new regularity result in [17], which gives

$\mathcal{L}^{(1)}: L^{2}\left(0, T ; L^{2}\left(\Gamma_{1}\right) \times H^{-1}\left(\Gamma_{1}\right)\right) \rightarrow C\left([0, T] ; H^{\frac{3}{2}}(\Omega) \times H^{\frac{1}{2}}(\Omega) \times L^{2}(\Omega)\right)$ continuously.

Combining (3.3) and (3.4) (at terminal time $T$ ) with (3.2), we thus deduce that the mapping

$$
\left(\mathbb{I}-\Pi^{*} \Pi\right) \mathcal{L}_{T} \mathcal{L}_{T}^{*} \Pi^{*}\left(\Pi \mathcal{L}_{T} \mathcal{L}_{T}^{*} \Pi^{*}\right)^{-1} \Pi \in \mathcal{L}\left(\mathbf{H}_{\gamma}\right),
$$

where $\mathbb{I}: \mathbf{H}_{\gamma} \rightarrow \mathbf{H}_{\gamma}$ denotes the identity.

Combining (3.2) and (3.5) thus gives

$$
\mathcal{L}_{T} \mathcal{L}_{T}^{*} \Pi^{*}\left(\Pi \mathcal{L}_{T} \mathcal{L}_{T}^{*} \Pi^{*}\right)^{-1} \Pi \in \mathcal{L}\left(\mathbf{H}_{\gamma}\right) .
$$

Step 1 . For arbitrary $\epsilon>0$ we select a $\bar{u}_{1} \in D\left(\mathcal{L}_{T}\right) \subset \mathcal{U}_{r+1}$, so that for arbitrary terminal state $\left[\omega_{0}^{T}, \omega_{1}^{T}, \theta_{0}^{T}\right] \in \mathbf{H}_{\gamma}$, the corresponding solution $\left[\omega^{(1)}(t), \omega_{t}^{(1)}(t), \theta^{(1)}(t)\right]$ to (1.1), with $\left[u_{1}, u_{2}, u_{3}\right] \equiv \bar{u}_{1}$ and zero initial data, satisfies

$$
\begin{aligned}
& \left\|\left[\begin{array}{c}
\omega^{(1)}(T)-\omega_{0}^{T} \\
\omega_{t}^{(1)}(T)-\omega_{1}^{T} \\
\theta^{(1)}(T)-\theta_{0}^{T}
\end{array}\right]+e^{\mathcal{A}_{\gamma} T}\left[\begin{array}{c}
\omega_{0} \\
\omega_{1} \\
\theta_{0}
\end{array}\right]\right\|_{\mathbf{H}_{\gamma}} \\
& \quad<\frac{\epsilon}{1+\left\|(\mathbb{I}-\Pi * \Pi) \mathcal{L}_{T} \mathcal{L}_{T}^{*} \Pi^{*}\left(\Pi \mathcal{L}_{T} \mathcal{L}_{T}^{*} \Pi^{*}\right)^{-1} \Pi\right\|_{\mathcal{L}\left(\mathbf{H}_{\gamma}\right)}}
\end{aligned}
$$

(where the fact that $(\mathbb{I}-\Pi * \Pi) \mathcal{L}_{T} \mathcal{L}_{T}^{*} \Pi^{*}\left(\Pi \mathcal{L}_{T} \mathcal{L}_{T}^{*} \Pi^{*}\right)^{-1} \Pi$ is due to (3.6)).

Step 2. We now select $\bar{u}_{2} \in D\left(\mathcal{L}_{T}\right)$ to be the "minimal norm steering control" with respect to the (partial) terminal state $\left[\omega_{0}^{T}-\omega^{(1)}(T), \omega_{1}^{T}-\omega_{t}^{(1)}(T)\right]$. That is to say, $\bar{u}_{2}$ satisfies

$$
\Pi \mathcal{L}_{T} \bar{u}_{2}+\Pi e^{\mathcal{A}_{\gamma} T}\left[\begin{array}{c}
\omega_{0} \\
\omega_{1} \\
\theta_{0}
\end{array}\right]=\left[\begin{array}{c}
\omega_{0}^{T}-\omega^{(1)}(T) \\
\omega_{1}^{T}-\omega_{t}^{(1)}(T)
\end{array}\right]
$$

and minimizes the functional $\frac{1}{2}\|\bar{u}\|_{\mathcal{U}_{s}}^{2}$, over all $\bar{u} \in \mathcal{U}_{s}$, which satisfies

$$
\Pi \mathcal{L}_{T} \bar{u}=\left[\begin{array}{c}
\omega_{0}^{T}-\omega^{(1)}(T) \\
\omega_{1}^{T}-\omega_{t}^{(1)}(T)
\end{array}\right]-\Pi e^{\mathcal{A}_{\gamma} T}\left[\begin{array}{c}
\omega_{0} \\
\omega_{1} \\
\theta_{0}
\end{array}\right] .
$$

(By Theorem 1.2. we know there exists at least one such $\bar{u}$.) By convex optimization theory and Lax-Milgram, the minimizer $\bar{u}_{2}$ can be given explicitly by

$$
\bar{u}_{2}=\mathcal{L}_{T}^{*} \Pi^{*}\left(\Pi \mathcal{L}_{T} \mathcal{L}_{T}^{*} \Pi^{*}\right)^{-1} \Pi\left(\left[\begin{array}{c}
\omega_{0}^{T}-\omega^{(1)}(T) \\
\omega_{1}^{T}-\omega_{t}^{(1)}(T) \\
\theta_{0}^{T}-\theta^{(1)}(T)
\end{array}\right]-e^{\mathcal{A}_{\gamma} T}\left[\begin{array}{c}
\omega_{0} \\
\omega_{1} \\
\theta_{0}
\end{array}\right]\right)
$$

(see (B.20) of [14, p. 288]). With this representation, we then have from (3.7) the norm bound

$$
\left\|\left(\mathbb{I}-\Pi^{*} \Pi\right) \mathcal{L}_{T} \bar{u}_{2}\right\|_{\mathbf{H}_{\gamma}} \leq \frac{\left\|\left(\mathbb{I}-\Pi^{*} \Pi\right) \mathcal{L}_{T} \mathcal{L}_{T}^{*} \Pi^{*}\left(\Pi \mathcal{L}_{T} \mathcal{L}_{T}^{*} \Pi^{*}\right)^{-1} \Pi\right\|_{\mathcal{L}\left(\mathbf{H}_{\gamma}\right)} \cdot \epsilon}{1+\left\|\left(\mathbb{I}-\Pi^{*} \Pi\right) \mathcal{L}_{T} \mathcal{L}_{T}^{*} \Pi^{*}\left(\Pi \mathcal{L}_{T} \mathcal{L}_{T}^{*} \Pi^{*}\right)^{-1} \Pi\right\|_{\mathcal{L}\left(\mathbf{H}_{\gamma}\right)}}
$$


Step 3 . Set the control $\bar{u}^{*}=\bar{u}_{1}+\bar{u}_{2}$. Consequently, there is the equality

$$
\begin{aligned}
\mathcal{L}_{T} \bar{u}^{*}+e^{\mathcal{A}_{\gamma} T}\left[\begin{array}{c}
\omega_{0} \\
\omega_{1} \\
\theta_{0}
\end{array}\right]= & \mathcal{L}_{T} \bar{u}_{1}+\mathcal{L}_{T} \bar{u}_{2}+e^{\mathcal{A}_{\gamma} T}\left[\begin{array}{c}
\omega_{0} \\
\omega_{1} \\
\theta_{0}
\end{array}\right]=\left[\begin{array}{c}
\omega_{0}^{T} \\
\omega_{1}^{T} \\
\theta^{(1)}(T)
\end{array}\right] \\
& +\left(\mathbb{I}-\Pi^{*} \Pi\right)\left[\mathcal{L}_{T} \bar{u}_{2}+e^{\mathcal{A}_{\gamma} T}\left[\begin{array}{c}
\omega_{0} \\
\omega_{1} \\
\theta_{0}
\end{array}\right]\right]
\end{aligned}
$$

Letting $\left[\omega^{*}, \omega_{t}^{*}, \theta^{*}\right]$ denote the solution of (1.1) corresponding to the chosen control $\bar{u}^{*}$, we then have from (3.11) that $\left[\omega^{*}(T), \omega_{t}^{*}(T)\right]=\left[\omega_{0}^{T}, \omega_{1}^{T}\right]$. Moreover, from (3.11), (3.7), and (3.10) we obtain the estimate

$$
\begin{aligned}
& \left\|\theta^{*}(T)-\theta_{0}^{T}\right\|_{L_{\sigma+\lambda}^{2}(\Omega)} \leq\left\|\left[\begin{array}{c}
0 \\
0 \\
\theta^{(1)}(T)-\theta_{0}^{T}
\end{array}\right]+\left(\mathbb{I}-\Pi^{*} \Pi\right)\left[e^{\mathcal{A}_{\gamma} T}\left[\begin{array}{c}
\omega_{0} \\
\omega_{1} \\
\theta_{0}
\end{array}\right]\right]\right\|_{\mathbf{H}_{\gamma}} \\
& \quad+\left\|\left(\mathbb{I}-\Pi^{*} \Pi\right) \mathcal{L}_{T} \bar{u}_{2}\right\|_{\mathbf{H}_{\gamma}} \\
& \quad<\frac{\epsilon}{1+\left\|\left(\mathbb{I}-\Pi^{*} \Pi\right) \mathcal{L}_{T} \mathcal{L}_{T}^{*} \Pi^{*}\left(\Pi \mathcal{L}_{T} \mathcal{L}_{T}^{*} \Pi^{*}\right)^{-1} \Pi\right\|_{\mathcal{L}\left(\mathbf{H}_{\gamma}\right)}+\left\|\left(\mathbb{I}-\Pi^{*} \Pi\right) \mathcal{L}_{T} \bar{u}_{2}\right\|_{\mathbf{H}_{\gamma}}<\epsilon .}
\end{aligned}
$$

Thus, the constructed control $\bar{u}^{*}=\left[u_{1}^{*}, u_{2}^{*}, u_{3}^{*}\right] \in \mathcal{U}_{r+1}$ satisfies the desired exactapproximate controllability property. Moreover, the Sobolev embedding theorem gives that $u_{3}^{*} \in C^{r}\left(\Sigma_{2, T}\right)$. This concludes the proof of Theorem 1.1.

\section{Appendix.}

Proposition 4.1. The operator $A_{R}-\frac{\sigma}{\eta}+\lambda \AA \AA G_{2} \gamma_{0}-\AA G_{1} \gamma_{0}$ is an element of $\mathcal{L}\left(L^{2}(\Omega),\left[D\left(\AA^{\frac{1}{2}}\right)\right]^{\prime}\right)$ and $\left(A_{R}-\frac{\sigma}{\eta}+\lambda \AA G_{2} \gamma_{0}-\AA G_{1} \gamma_{0}\right)^{*}=A_{D}\left(I-D \gamma_{0}\right)$ as elements of $\mathcal{L}\left(D\left(\AA^{\frac{1}{2}}\right), L^{2}(\Omega)\right)$.

Proof. For every $\vartheta \in D\left(A_{R}\right)$ and $\varpi \in D\left(\AA^{\frac{1}{2}}\right)$, we have

$$
\begin{aligned}
& \left\langle\left(A_{R}-\frac{\sigma}{\eta}+\lambda \AA G_{2} \gamma_{0}-\AA G_{1} \gamma_{0}\right) \vartheta, \varpi\right\rangle\left[D\left(\AA^{\frac{1}{2}}\right)\right]^{\prime} \times D \mid\left(\AA^{\frac{1}{2}}\right) \\
= & \left.\left.(-\Delta \vartheta, \varpi)_{L^{2}(\Omega)}+\left\langle\lambda \AA G_{2} \gamma_{0} \vartheta, \varpi\right\rangle_{D}\left(\AA^{\frac{1}{2}}\right)\right]^{\prime} \times D\left(\AA^{\frac{1}{2}}\right)-\left\langle\AA G_{1} \gamma_{0} \vartheta, \varpi\right\rangle_{D}\left(\AA^{\frac{1}{2}}\right)\right]^{\prime} \times D\left(\AA^{\frac{1}{2}}\right) \\
= & (\nabla \vartheta, \nabla \varpi)_{L^{2}(\Omega)}-\left(\frac{\partial \vartheta}{\partial \nu}, \varpi\right)_{L^{2}\left(\Gamma_{1}\right)}+\lambda\left(\gamma_{0} \vartheta, G_{2}^{*} \AA \varpi\right)_{L^{2}\left(\Gamma_{1}\right)}-\left(\gamma_{0} \vartheta, G_{1}^{*} \AA \varpi\right)_{L^{2}\left(\Gamma_{1}\right)} \\
& (\text { after the use of Green's formula and the taking of adjoints }) \\
= & (\nabla \vartheta, \nabla \varpi)_{L^{2}(\Omega)}-\left(\gamma_{0} \vartheta, G_{1}^{*} \AA \varpi\right)_{L^{2}\left(\Gamma_{1}\right)}=(\vartheta,-\Delta \varpi)_{L^{2}(\Omega)}
\end{aligned}
$$

(after one more use of Green's theorem and the characterization (1.23)) $=\left(\vartheta, A_{D}\left(I-D \gamma_{0}\right) \varpi\right)_{L^{2}(\Omega)}$.

As $D\left(A_{R}\right)$ is dense in $L^{2}(\Omega)$, this equality proves the assertion. 
Lemma 4.2. The Hilbert space adjoint $\mathcal{A}_{\gamma}^{*}$ of $\mathcal{A}_{\gamma}$, as defined in (1.36), is given to be

$$
\begin{aligned}
& \mathcal{A}_{\gamma}^{*}=\left(\begin{array}{ccc}
\mathbf{I} & \mathbf{0} & \mathbf{0} \\
\mathbf{0} & P_{\gamma}^{-1} & \mathbf{0} \\
\mathbf{0} & \mathbf{0} & \mathbf{I}
\end{array}\right)\left(\begin{array}{ccc}
\mathbf{0} & -\mathbf{I} & \mathbf{0} \\
\AA & \mathbf{0} & -\alpha(\mathbf{\beta}) \\
\mathbf{0} & \frac{\alpha}{\beta} A_{D}\left(\mathbf{I}-D \gamma_{0}\right) & -\frac{\eta}{\beta} A_{R}
\end{array}\right), \\
& \text { with } D\left(\mathcal{A}_{\gamma}^{*}\right)=\left\{\left[\phi_{0}, \phi_{1}, \psi_{0}\right] \in D\left(\AA^{\frac{1}{2}}\right) \times D\left(\AA^{\frac{1}{2}}\right) \times D\left(A_{R}\right)\right. \\
& \text { such that } \left.\AA \phi_{0}+\alpha \AA G_{1} \gamma_{0} \psi_{0} \in H_{\Gamma_{0}, \gamma}^{-1}(\Omega)\right\}
\end{aligned}
$$

(above, (\$) is the same denotation made in (1.35)).

Proof. We define $\mathcal{S} \subseteq \mathbf{H}_{\gamma}$ to be

$$
\begin{aligned}
\mathcal{S} \equiv & \left\{\left[\phi_{0}, \phi_{1}, \psi_{0}\right] \in D\left(\AA^{\frac{1}{2}}\right) \times D\left(\AA^{\frac{1}{2}}\right) \times D\left(A_{R}\right)\right. \\
& \text { such that } \left.\AA \omega_{1}+\alpha \AA G_{1} \gamma_{0} \theta \in H_{\Gamma_{0}, \gamma}^{-1}(\Omega)\right\}
\end{aligned}
$$

and proceed to show that $D\left(\mathcal{A}_{\gamma}^{*}\right)=\mathcal{S}$. Indeed, if $\left[\omega_{1}, \omega_{2}, \theta\right] \in D\left(\mathcal{A}_{\gamma}\right)$ and $\left[\widetilde{\omega}_{1}, \widetilde{\omega}_{2}, \widetilde{\theta}\right] \in$ $\mathcal{S}$, we have by using (1.36)

$$
\begin{aligned}
& \left(\mathcal{A}_{\gamma}\left[\begin{array}{c}
\omega_{1} \\
\omega_{2} \\
\theta
\end{array}\right],\left[\begin{array}{c}
\widetilde{\omega}_{1} \\
\widetilde{\omega}_{2} \\
\widetilde{\theta}
\end{array}\right]\right)_{\mathbf{H}_{\gamma}} \\
& =\left(\AA^{\frac{1}{2}} \omega_{2}, \AA^{\frac{1}{2}} \widetilde{\omega}_{1}\right)_{L^{2}(\Omega)} \\
& +\left(P_{\gamma}^{-1}\left(-\AA \omega_{1}+\alpha A_{R} \theta-\frac{\alpha \sigma}{\eta} \theta-\alpha \AA G_{1} \gamma_{0} \theta+\alpha \lambda \AA G_{2} \gamma_{0} \theta\right), \widetilde{\omega}_{2}\right)_{H_{\Gamma_{0}, \gamma}^{1}(\Omega)} \\
& -\left(\alpha A_{D}\left(I-D \gamma_{0}\right) \omega_{2}, \widetilde{\theta}\right)_{L^{2}(\Omega)}-\frac{\eta \beta}{\beta}\left(A_{R} \theta, \widetilde{\theta}\right)_{L^{2}(\Omega)}
\end{aligned}
$$

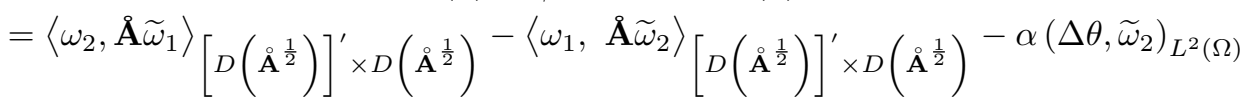

$$
\begin{aligned}
& -\alpha\left\langle\AA G_{1} \gamma_{0} \theta, \widetilde{\omega}_{2}\right\rangle\left[D\left(\AA^{\frac{1}{2}}\right)\right]^{\prime} \times D\left(\AA^{\frac{1}{2}}\right)+\alpha \lambda\left\langle\AA G_{2} \gamma_{0} \theta, \widetilde{\omega}_{2}\right\rangle\left[D\left(\AA^{\frac{1}{2}}\right)\right]^{\prime} \times D\left(\AA^{\frac{1}{2}}\right) \\
& +\alpha\left(\Delta \omega_{2}, \widetilde{\theta}\right)_{L^{2}(\Omega)}-\beta\left(\theta, \frac{\eta}{\beta} A_{R} \tilde{\theta}\right)_{L^{2}(\Omega)} \\
& \text { (after using the equality posted in (1.32)) }
\end{aligned}
$$

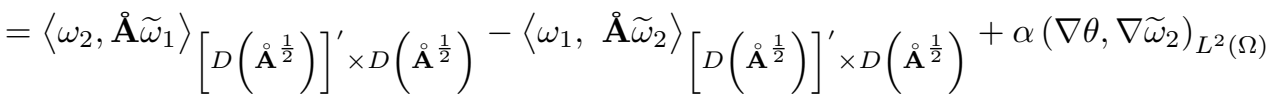

$$
\begin{aligned}
& -\alpha\left(\frac{\partial \theta}{\partial \nu}, \gamma_{0} \widetilde{\omega}_{2}\right)_{L^{2}\left(\Gamma_{1}\right)}-\alpha\left(\gamma_{0} \theta, \frac{\partial \widetilde{\omega}_{2}}{\partial \nu}\right)_{L^{2}\left(\Gamma_{1}\right)}-\alpha \lambda\left(\gamma_{0} \theta, \gamma_{0} \widetilde{\omega}_{2}\right)_{L^{2}\left(\Gamma_{1}\right)} \\
& -\alpha\left(\nabla \omega_{2}, \nabla \widetilde{\theta}\right)_{L^{2}(\Omega)}+\alpha\left(\frac{\partial \omega_{2}}{\partial \nu}, \gamma_{0} \widetilde{\theta}\right)_{L^{2}\left(\Gamma_{1}\right)}-\beta\left(\theta, \frac{\eta}{\beta} A_{R} \widetilde{\theta}\right)_{L^{2}\left(\Gamma_{1}\right)} \\
& \text { (after using Green's theorem and (1.23)) } \\
& =-\left(\AA^{\frac{1}{2}} \omega_{1}, \AA^{\frac{1}{2}} \widetilde{\omega}_{2}\right)_{L^{2}(\Omega)}+\left\langle\omega_{2}, \AA \widetilde{\omega}_{1}\right\rangle_{\left[D\left(\AA^{\frac{1}{2}}\right)\right]^{\prime} \times D\left(\AA^{\frac{1}{2}}\right)}+\beta\left(\theta,-\frac{\alpha}{\beta} \Delta \widetilde{\omega}_{2}\right)_{L^{2}(\Omega)}
\end{aligned}
$$




$$
\begin{aligned}
& +\alpha\left(\omega_{2}, \Delta \widetilde{\theta}\right)_{L^{2}(\Omega)}-\alpha\left(\gamma_{0} \omega_{2}, \frac{\partial \widetilde{\theta}}{\partial \nu}\right)_{L^{2}\left(\Gamma_{1}\right)}+\alpha\left(G_{1}^{*} \AA \omega_{2}, \gamma_{0} \widetilde{\theta}\right)_{L^{2}\left(\Gamma_{1}\right)} \\
& -\beta\left(\theta, \frac{\eta}{\beta} A_{R} \widetilde{\theta}\right)_{L^{2}\left(\Gamma_{1}\right)} \\
& =-\left(\AA^{\frac{1}{2}} \omega_{1}, \AA^{\frac{1}{2}} \widetilde{\omega}_{2}\right)_{L^{2}(\Omega)}+\left\langle\omega_{2}, \AA \widetilde{\mathbf{\omega}}_{1}\right\rangle\left[D\left(\AA^{\frac{1}{2}}\right)\right]^{\prime} \times D\left(\AA^{\frac{1}{2}}\right) \\
& +\alpha\left(\omega_{2},-A_{R} \widetilde{\theta}+\frac{\sigma}{\eta} \widetilde{\theta}\right)_{L^{2}(\Omega)}-\alpha \lambda\left(G_{2}^{*} \AA \omega_{2}, \gamma_{0} \widetilde{\theta}\right)_{L^{2}\left(\Gamma_{1}\right)} \\
& +\alpha\left\langle\omega_{2}, \AA G_{1} \gamma_{0} \widetilde{\theta}\right\rangle\left[{ }_{D}\left(\AA^{\frac{1}{2}}\right)\right]^{\prime} \times D\left(\AA^{\frac{1}{2}}\right){ }^{+\beta}\left(\theta, \frac{\alpha}{\beta} A_{D}\left(I-D \gamma_{0}\right) \widetilde{\omega}_{2}\right)_{L^{2}(\Omega)} \\
& -\beta\left(\theta, \frac{\eta}{\beta} A_{R} \tilde{\theta}\right)_{L^{2}\left(\Gamma_{1}\right)} \\
& =-\left(\AA^{\frac{1}{2}} \omega_{1}, \AA^{\frac{1}{2}} \widetilde{\omega}_{2}\right)_{L^{2}(\Omega)} \\
& +\left(P_{\gamma}^{\frac{1}{2}} \omega_{2}, P_{\gamma}^{\frac{1}{2}} P_{\gamma}^{-1}\left[\AA \widetilde{\omega}_{1}+\alpha\left(-A_{R} \widetilde{\theta}+\frac{\sigma}{\eta} \widetilde{\theta}+\AA G_{1} \gamma_{0} \widetilde{\theta}-\lambda \AA G_{2} \gamma_{0} \widetilde{\theta}\right)\right]\right)_{L^{2}(\Omega)} \\
& +\beta\left(\theta, \frac{\alpha}{\beta} A_{D}\left(I-D \gamma_{0}\right) \widetilde{\omega}_{2}\right)_{L^{2}(\Omega)}-\beta\left(\theta, \frac{\eta}{\beta} A_{R} \widetilde{\theta}\right)_{L^{2}\left(\Gamma_{1}\right)} \\
& \text { (after again using } \left.(1.32),(1.23) \text {, and the fact that }\left[\widetilde{\omega}_{1}, \widetilde{\omega}_{2}, \widetilde{\theta}\right] \in \mathcal{S}\right) \\
& =\left(\left[\begin{array}{c}
\omega_{1} \\
\omega_{2} \\
\theta
\end{array}\right], \mathcal{T}\left[\begin{array}{c}
\widetilde{\omega}_{1} \\
\widetilde{\omega}_{2} \\
\widetilde{\theta}
\end{array}\right]\right)_{\mathbf{H}_{\gamma}},
\end{aligned}
$$

where

$$
\mathcal{T} \equiv\left(\begin{array}{ccc}
\mathbf{I} & \mathbf{0} & \mathbf{0} \\
\mathbf{0} & P_{\gamma}^{-1} & \mathbf{0} \\
\mathbf{0} & \mathbf{0} & \mathbf{I}
\end{array}\right)\left(\begin{array}{ccc}
\mathbf{0} & -\mathbf{I} & \mathbf{0} \\
\AA & \mathbf{0} & -\alpha(\mathbf{p}) \\
\mathbf{0} & \frac{\alpha}{\beta} A_{D}\left(\mathbf{I}-D \gamma_{0}\right) & -\frac{\eta}{\beta} A_{R}
\end{array}\right)
$$

Thus,

$$
\mathcal{S} \subseteq D\left(\mathcal{A}_{\gamma}^{*}\right) \text { and }\left.\mathcal{A}_{\gamma}^{*}\right|_{\mathcal{S}}=\mathcal{T}
$$

To show the opposite containment, one can straightforwardly compute the inverse $\mathcal{A}_{\gamma}^{-1} \in \mathcal{L}\left(\mathbf{H}_{\gamma}, D\left(\mathcal{A}_{\gamma}\right)\right)$ as

$(4.2) \mathcal{A}_{\gamma}^{-1}=\left(\begin{array}{ccc}-\frac{\alpha^{2}}{\eta} \AA^{-1}(\boldsymbol{\beta}) A_{R}^{-1} A_{D}\left(I-D \gamma_{0}\right) & -\AA^{-1} P_{\gamma} & -\frac{\alpha \beta}{\eta} \AA^{-1}(\boldsymbol{\beta}) A_{R}^{-1} \\ \mathbf{I} & \mathbf{0} & \mathbf{0} \\ -\frac{\alpha}{\eta} A_{R}^{-1} A_{D}\left(I-D \gamma_{0}\right) & \mathbf{0} & -\frac{\beta}{\eta} A_{R}^{-1}\end{array}\right)$.

In turn, one can use this quantity and Proposition 4.1 to compute the Hilbert space 
adjoint $\left(\mathcal{A}_{\gamma}^{*}\right)^{-1}$ of $\mathcal{A}_{\gamma}^{-1}$ as

$$
\left(\mathcal{A}_{\gamma}^{*}\right)^{-1}=\left(\begin{array}{ccc}
-\frac{\alpha^{2}}{\eta} \AA^{-1}(\boldsymbol{\beta}) A_{R}^{-1} A_{D}\left(I-D \gamma_{0}\right) & \AA^{-1} P_{\gamma} & -\frac{\alpha \beta}{\eta} \AA^{-1}(\boldsymbol{\beta}) A_{R}^{-1} \\
-\mathbf{I} & \mathbf{0} & \mathbf{0} \\
-\frac{\alpha}{\eta} A_{R}^{-1} A_{D}\left(I-D \gamma_{0}\right) & \mathbf{0} & -\frac{\beta}{\eta} A_{R}^{-1}
\end{array}\right)
$$

With this quantity in hand, we then have that for arbitrary $\left[\phi_{0}, \phi_{1}, \psi_{0}\right] \in D\left(\mathcal{A}_{\gamma}^{*}\right)$ and corresponding

$$
\begin{gathered}
{\left[\begin{array}{l}
\omega_{0} \\
\omega_{1} \\
\theta_{0}
\end{array}\right]=\mathcal{A}_{\gamma}^{*}\left[\begin{array}{l}
\phi_{0} \\
\phi_{1} \\
\psi_{0}
\end{array}\right] \in \mathbf{H}_{\gamma},} \\
{\left[\begin{array}{l}
\phi_{0} \\
\phi_{1} \\
\psi_{0}
\end{array}\right]=\left(\mathcal{A}_{\gamma}^{*}\right)^{-1}\left[\begin{array}{l}
\omega_{0} \\
\omega_{1} \\
\theta_{0}
\end{array}\right]} \\
=\left[\begin{array}{c}
-\frac{\alpha^{2}}{\eta} \AA^{-1}(\boldsymbol{\beta}) A_{R}^{-1} A_{D}\left(I-D \gamma_{0}\right) \omega_{0}+\AA^{-1} P_{\gamma} \omega_{1}-\frac{\alpha \beta}{\eta} \AA^{-1}(\boldsymbol{\beta}) A_{R}^{-1} \theta_{0} \\
-\omega_{0} \\
-\frac{\alpha}{\eta} A_{R}^{-1} A_{D}\left(I-D \gamma_{0}\right) \omega_{0}-\frac{\beta}{\eta} A_{R}^{-1} \theta_{0}
\end{array}\right] .
\end{gathered}
$$

A fortiori then, $\left[\phi_{0}, \phi_{1}\right] \in\left[D\left(\AA^{\frac{1}{2}}\right)\right]^{2}$ and $\psi_{0} \in D\left(A_{R}\right)$. Moreover, (4.4) and the definition of the operator (\$) in (1.35) gives

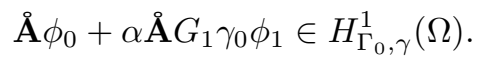

Thus, $D\left(\mathcal{A}_{\gamma}^{*}\right) \subseteq \mathcal{S}$, and this combined with (4.1) concludes Lemma 4.2.

Proposition 4.3. For arbitrary terminal data $\left[\phi_{0}, \phi_{1}, \psi_{0}\right] \in \mathbf{H}_{\gamma}$, the solution $\left[\phi, \phi_{t}, \psi\right]$ to (1.51) has the following additional regularity:

$$
\left\|\phi_{t t}\right\|_{L^{\infty}\left(0, T ;\left[D\left(\mathbf{A}^{\circ \frac{1}{2}} P_{\gamma}^{-1}\right)\right]^{\prime}\right)} \leq C\left\|\left[\phi_{0}, \phi_{1}, \psi_{0}\right]\right\|_{\mathbf{H}_{\gamma}},
$$

where $\AA^{\frac{1}{2}} P_{\gamma}^{-1}$ is taken as a closed and densely defined operator, $\AA^{\frac{1}{2}} P_{\gamma}^{-1}: D\left(\AA^{\frac{1}{2}} P_{\gamma}^{-1}\right) \subset$ $L^{2}(\Omega) \rightarrow L^{2}(\Omega)$, with $D\left(\AA^{\frac{1}{2}} P_{\gamma}^{-1}\right)=\left\{\varphi \in L^{2}(\Omega): P_{\gamma}^{-1} \varphi \in D\left(\AA^{\frac{1}{2}}\right)\right\}$.

Proof. For terminal data $\left[\phi_{0}, \phi_{1}, \psi_{0}\right] \in D\left(\mathcal{A}_{\gamma}^{*}\right)$, we have for all $\varpi \in L^{1}\left(0, T ; D\left(\AA^{\frac{1}{2}} P_{\gamma}^{-1}\right)\right)$, upon using the abstract equation (1.52), the characterizations in (1.23), the fact that $P_{\gamma}^{-1} \varpi \in L^{1}\left(0, T ; D\left(\AA^{\frac{1}{2}}\right) \cap D\left(A_{N}\right)\right)$ (recall the definition of $A_{N}$ in (1.16) and $P_{\gamma}$ in (1.24)), and $\left.\frac{\partial \psi}{\partial \nu}\right|_{\Gamma}=-\left.\lambda \psi\right|_{\Gamma}$, that

$$
\begin{aligned}
& \int_{0}^{T}\left(\varpi, \phi_{t t}\right)_{L^{2}(\Omega)} d t=\int_{0}^{T}\left(\varpi, P_{\gamma}^{-1}\left[-\AA \AA \AA \phi-\alpha \AA G_{1} \gamma_{0} \psi+\alpha \lambda \AA G_{2} \gamma_{0} \psi-\alpha \Delta \psi\right]\right)_{L^{2}(\Omega)} d t \\
= & \int_{0}^{T}\left[-\left(\AA^{\frac{1}{2}} P_{\gamma}^{-1} \varpi, \AA^{\frac{1}{2}} \phi\right)_{L^{2}(\Omega)}-\alpha\left(G_{1}^{*} \AA P_{\gamma}^{-1} \varpi, \gamma_{0} \psi\right)_{L^{2}\left(\Gamma_{1}\right)}\right.
\end{aligned}
$$




$$
\begin{aligned}
& \left.+\alpha \lambda\left(G_{2}^{*} \AA P_{\gamma}^{-1} \varpi, \gamma_{0} \psi\right)_{L^{2}\left(\Gamma_{1}\right)}-\alpha\left(P_{\gamma}^{-1} \varpi, \Delta \psi\right)_{L^{2}(\Omega)}\right] d t \\
= & \int_{0}^{T}\left[\left(-\AA^{\frac{1}{2}} P_{\gamma}^{-1} \varpi, \AA^{\frac{1}{2}} \phi\right)_{L^{2}(\Omega)}+\alpha\left(P_{\gamma}^{-1} \varpi, \frac{\partial \psi}{\partial \nu}\right)_{L^{2}\left(\Gamma_{1}\right)}-\alpha\left(P_{\gamma}^{-1} \varpi, \Delta \psi\right)_{L^{2}(\Omega)}\right] d t \\
= & \int_{0}^{T}\left[\left(-\AA^{\frac{1}{2}} P_{\gamma}^{-1} \varpi, \AA^{\frac{1}{2}} \phi\right)_{L^{2}(\Omega)}+\alpha\left(\nabla P_{\gamma}^{-1} \varpi, \nabla \psi\right)_{L^{2}(\Omega)}\right] d t \\
= & \int_{0}^{T}\left[\left(-\AA^{\frac{1}{2}} P_{\gamma}^{-1} \varpi, \AA^{\frac{1}{2}} \phi\right)_{L^{2}(\Omega)}-\alpha\left(\Delta P_{\gamma}^{-1} \varpi, \psi\right)_{L^{2}(\Omega)}\right] d t .
\end{aligned}
$$

Estimating the far side of this expression by using the fact that $P_{\gamma}^{-1} \in \mathcal{L}\left(L^{2}(\Omega), D\left(A_{N}\right)\right)$, followed by the contraction of the semigroup $\left\{e^{\mathcal{A}_{\gamma}^{*} t}\right\}_{t \geq 0}$, one has the estimate

$$
\int_{0}^{T}\left(\varpi, \phi_{t t}\right)_{L^{2}(\Omega)} d t \leq C\left\|\left[\begin{array}{c}
\phi_{0} \\
\phi_{1} \\
\psi_{0}
\end{array}\right]\right\|_{\mathbf{H}_{\gamma}}\|\varpi\|_{L^{1}\left(0, T ; D\left(\AA^{\frac{1}{2}} P_{\gamma}^{-1}\right)\right)} d t .
$$

A density argument concludes the proof.

Proposition 4.4. If $\left[\phi, \phi_{t}, \psi\right]$ denotes the solution to (1.51), corresponding to terminal data $\left[\phi_{0}, \phi_{1}, \psi_{0}\right]$, we have the following estimates.

1. The map $\left[\phi_{0}, \phi_{1}, \psi_{0}\right] \rightarrow \Delta \phi$ is an element of $\mathcal{L}\left(\mathbf{H}_{\gamma}, L^{2}\left(0, T ;\left[H^{1}(\Omega)\right]^{\prime}\right)\right)$, with the norm bound

$$
\|\Delta \phi\|_{L^{2}\left(0, T ;\left[H^{1}(\Omega)\right]^{\prime}\right)} \leq \text { I.o.t. }\left(\phi, \phi_{t}, \psi\right) .
$$

2. The map $\left[\phi_{0}, \phi_{1}, \psi_{0}\right] \rightarrow\left[\Delta \phi_{t}, \psi_{t}\right]$ is an element of $\mathcal{L}\left(D\left(\mathcal{L}_{T}^{*}\right),\left[L^{2}\left(0, T ;\left[H^{\frac{3}{2}-\epsilon}(\Omega)\right]^{\prime}\right)\right]^{2}\right)$, with the norm bound

$$
\left\|\left[\Delta \phi_{t}, \psi_{t}\right]\right\|\left[L_{L^{2}}\left(0, T ;\left[H^{\frac{3}{2}-\epsilon}(\Omega)\right]^{\prime}\right)\right]^{2} \leq C\left\|\nabla \phi_{t}\right\|_{L^{2}\left(0, T ; L^{2}\left(\Gamma_{1}\right)\right)}+\text { I.o.t. }\left(\phi, \phi_{t}, \psi\right) .
$$

Proof of (i). For all $\varpi \in L^{2}\left(0, T ; H^{1}(\Omega)\right)$, we easily have

$$
\begin{aligned}
& \int_{0}^{T}(\Delta \phi, \varpi)_{L^{2}(\Omega)} d t=-\int_{0}^{T}(\nabla \phi, \nabla \varpi)_{L^{2}(\Omega)} d t+\int_{0}^{T}\left(\frac{\partial \phi}{\partial \nu}, \varpi\right)_{L^{2}\left(\Gamma_{1}\right)} d t \\
\leq & C \int_{0}^{T}\left[\|\nabla \phi\|_{L^{2}(\Omega)}\|\nabla \varpi\|_{L^{2}(\Omega)}+\|\phi\|_{H^{\frac{3}{2}+\epsilon}(\Omega)}\|\varpi\|_{H^{1}(\Omega)}\right] d t \\
\leq & C\|\phi\|_{L^{2}\left(0, T ; H^{\frac{3}{2}+\epsilon}(\Omega)\right.}\|\varpi\|_{L^{2}\left(0, T ; H^{1}(\Omega)\right)},
\end{aligned}
$$

and this estimate gives the asserted result.

Proof of (ii). If $\left[\phi_{0}, \phi_{1}, \psi_{0}\right] \in D\left(\mathcal{A}_{\gamma}^{*}\right)$, then $\left[\phi, \phi_{t}, \psi\right] \in C\left([0, T] ; D\left(\mathcal{A}_{\gamma}^{*}\right)\right) \cap C^{1}([0, T]$; $\left.\mathbf{H}_{\gamma}\right)$, and so in particular $\Delta \phi_{t} \in L^{2}\left(0, T ; L^{2}(\Omega)\right)$. Taking the $L^{2}$-inner product with respect to arbitrary $\varpi \in L^{2}\left(0, T ; H^{\frac{3}{2}-\epsilon}(\Omega)\right)$, we have upon the use of Green's theorem and the definition of $A_{R}$ in (1.14) that 


$$
\begin{aligned}
& -\int_{0}^{T}\left(\Delta \phi_{t}, \varpi\right)_{L^{2}(\Omega)} d t \\
= & \int_{0}^{T}\left(A_{R}^{\frac{1}{2}} \phi_{t}, A_{R}^{\frac{1}{2}} \varpi\right)_{L^{2}(\Omega)} d t \\
& -\int_{0}^{T}\left[\lambda\left(\phi_{t}, \varpi\right)_{L^{2}(\Gamma)}+\frac{\sigma}{\eta}\left(\phi_{t}, \varpi\right)_{L^{2}(\Omega)}+\left(\frac{\partial \phi_{t}}{\partial \nu}, \varpi\right)_{L^{2}\left(\Gamma_{1}\right)}\right] d t \\
= & \int_{0}^{T}\left(A_{R}^{\frac{1}{4}+\frac{\epsilon}{2}} \phi_{t}, A_{R}^{\frac{3}{4}-\frac{\epsilon}{2}} \varpi\right)_{L^{2}(\Omega)} d t \\
& -\int_{0}^{T}\left[\lambda\left(\phi_{t}, \varpi\right)_{L^{2}(\Gamma)}+\frac{\sigma}{\eta}\left(\phi_{t}, \varpi\right)_{L^{2}(\Omega)}+\left(\frac{\partial \phi_{t}}{\partial \nu}, \varpi\right)_{L^{2}\left(\Gamma_{1}\right)}\right] d t \\
\leq & \int_{0}^{T}\left(\left\|A_{R}^{\frac{1}{4}+\frac{\epsilon}{2}} \phi_{t}\right\|_{L^{2}(\Omega)}\left\|A_{R}^{\frac{3}{4}-\frac{\epsilon}{2}} \varpi\right\|_{L^{2}(\Omega)}\right. \\
& \left.\quad+\left(C\left\|\nabla \phi_{t}\right\|_{L^{2}\left(0, T ; L^{2}\left(\Gamma_{1}\right)\right)}+\text { I.o.t. }\left(\phi, \phi_{t}, \psi\right)\right)\|\varpi\|_{H^{\frac{1}{2}+\epsilon}(\Omega)}\right) d t \\
\leq & \left.\left(C\left\|\nabla \phi_{t}\right\|_{L^{2}\left(0, T ; L^{2}\left(\Gamma_{1}\right)\right)}+\text { I.o.t. }\left(\phi, \phi_{t}, \psi\right)\right)\|\varpi\|_{L^{2}\left(0, T ; H^{\frac{3}{2}-\epsilon}(\Omega)\right.}\right)
\end{aligned}
$$

Moreover, as $\left[\phi_{0}, \phi_{1}, \psi_{0}\right] \in D\left(\mathcal{A}_{\gamma}^{*}\right)$, we can take the $L^{2}$-inner product of $\psi_{t}$ with arbitrary $\varpi \in L^{2}\left(0, T ; H^{\frac{3}{2}-\epsilon}(\Omega)\right)$ and use (1.53) and (4.11) to obtain

$$
\begin{aligned}
& \int_{0}^{T}\left(\psi_{t}, \varpi\right)_{L^{2}(\Omega)} d t=\beta^{-1} \int_{0}^{T}\left(\eta A_{R} \psi+\alpha \Delta \phi_{t}, \varpi\right)_{L^{2}(\Omega)} d t \\
= & \beta^{-1} \int_{0}^{T}\left[\left(\eta A_{R}^{\frac{1}{4}+\frac{\epsilon}{2}} \psi, A_{R}^{\frac{3}{4}-\frac{\epsilon}{2}} \varpi\right)_{L^{2}(\Omega)}+\left(\alpha \Delta \phi_{t}, \varpi\right)_{L^{2}(\Omega)}\right] d t \\
\leq & \left.\left(C\left\|\nabla \phi_{t}\right\|_{L^{2}\left(0, T ; L^{2}\left(\Gamma_{1}\right)\right)}+\text { I.o.t. }\left(\phi, \phi_{t}, \psi\right)\right)\|\varpi\|_{L^{2}\left(0, T ; H^{\frac{3}{2}-\epsilon}(\Omega)\right.}\right)
\end{aligned}
$$

Having obtained estimates (4.11) and (4.12) with smooth data $\left[\phi_{0}, \phi_{1}, \psi_{0}\right]$, a density argument (see Remark 1.8) and a recollection of the form of the adjoint $\mathcal{L}_{T}^{*}$ in (1.55) will allow us to obtain the norm bound (4.9) for all terminal data in $D\left(\mathcal{L}_{T}^{*}\right)$.

LEMma 4.5. Concerning the component $\widehat{\phi}$ of the solution $\left[\widehat{\phi}, \widehat{\phi}_{t}, \widehat{\psi}\right]$ of $(2.9)$, one has that $\left.\Delta \widehat{\phi}\right|_{\Gamma_{0}} \in L^{2}\left(0, T ; L^{2}\left(\Gamma_{0}\right)\right)$ with the following estimate valid for all $s$ and $\tau \in[0, T]:$

$$
\int_{s}^{\tau}\|\Delta \widehat{\phi}\|_{L^{2}\left(\Gamma_{0}\right)}^{2} d t \leq C_{0}\left(\int_{0}^{T} E_{\widehat{\phi}}(t) d t+E_{\widehat{\phi}}(s)+E_{\widehat{\phi}}(\tau)\right)+\text { I.o.t. }(\widehat{\phi}, \widehat{\phi} t, \widehat{\psi}) .
$$

Proof. So as to obtain the inequality (4.13), we multiply the first equation of (2.15) by the quantity $\bar{m} \cdot \nabla \widehat{\phi}$, where $\bar{m}(x, y) \equiv\left[m_{1}(x, y), m_{2}(x, y)\right]$ is a $\left[C^{2}(\bar{\Omega})\right]^{2}$ vector field, ${ }^{1}$ which satisfies

$$
\left.\bar{m}\right|_{\Gamma}= \begin{cases}{\left[\nu_{1}, \nu_{2}\right]} & \text { on } \Gamma_{0} \\ 0 & \text { on } \Gamma_{1}\end{cases}
$$

\footnotetext{
${ }^{1}$ Here we make use of the fact that $\Gamma_{0}$ and $\Gamma_{1}$ are separated.
} 
and follow this by an integration from $s$ to $\tau$; i.e., we will work with the equation

$$
\begin{aligned}
\int_{s}^{\tau} & \left(\widehat{\phi}_{t t}-\gamma \Delta \widehat{\phi}_{t t}+\Delta^{2} \widehat{\phi}, \bar{m} \cdot \nabla \widehat{\phi}\right)_{L^{2}(\Omega)} d t \\
& =\int_{s}^{\tau}\left(c_{0} \widehat{\psi}+c_{1} \widehat{\psi}_{t}+c_{2} \widehat{\phi}+c_{3} \widehat{\phi}_{t}+c_{4} \Delta \widehat{\phi}, \bar{m} \cdot \nabla \widehat{\phi}\right)_{L^{2}(\Omega)} d t .
\end{aligned}
$$

To handle the left-hand side of (4.15), perform the following steps.

(i) First,

$$
\begin{aligned}
& \int_{s}^{\tau}\left(\widehat{\phi}_{t t}, \bar{m} \cdot \nabla \widehat{\phi}\right)_{L^{2}(\Omega)} d t=\left.\left(\widehat{\phi}_{t}, \bar{m} \cdot \nabla \widehat{\phi}\right)_{L^{2}(\Omega)}\right|_{s} ^{\tau}-\int_{s}^{\tau}\left(\widehat{\phi}_{t}, \bar{m} \cdot \nabla \widehat{\phi}_{t}\right)_{L^{2}(\Omega)} d t \\
= & \left.\left(\widehat{\phi}_{t}, \bar{m} \cdot \nabla \widehat{\phi}\right)_{L^{2}(\Omega)}\right|_{s} ^{\tau}-\frac{1}{2} \int_{s}^{\tau} \int_{\Omega} \operatorname{div}\left(\widehat{\phi}_{t}^{2} \bar{m}\right) d t d \Omega \\
& +\frac{1}{2} \int_{s}^{\tau} \int_{\Omega} \widehat{\phi}_{t}^{2}\left[m_{1 x}+m_{2 y}\right] d t d \Omega \\
= & \left.\left(\widehat{\phi}_{t}, \bar{m} \cdot \nabla \widehat{\phi}\right)_{L^{2}(\Omega)}\right|_{s} ^{\tau}+\frac{1}{2} \int_{s}^{\tau} \int_{\Omega} \widehat{\phi}_{t}^{2}\left[m_{1 x}+m_{2 y}\right] d t d \Omega,
\end{aligned}
$$

after making use of the divergence theorem and the fact that $\widehat{\phi}_{t}=0$ on $\Gamma_{0}$.

(ii) Next,

$$
\begin{aligned}
& \int_{s}^{\tau}\left(-\Delta \widehat{\phi}_{t t}, \bar{m} \cdot \nabla \widehat{\phi}\right)_{L^{2}(\Omega)} d t=\left.\left(\nabla \widehat{\phi}_{t}, \nabla(\bar{m} \cdot \nabla \widehat{\phi})\right)_{L^{2}(\Omega)}\right|_{s} ^{\tau} \\
& -\int_{s}^{\tau}\left(\nabla \widehat{\phi}_{t}, \nabla\left(\bar{m} \cdot \nabla \widehat{\phi}_{t}\right)\right)_{L^{2}(\Omega)} d t \\
= & \left.\left(\nabla \widehat{\phi}_{t}, \nabla(\bar{m} \cdot \nabla \widehat{\phi})\right)_{L^{2}(\Omega)}\right|_{s} ^{\tau}-\frac{1}{2} \int_{s}^{\tau} \int_{\Omega} \operatorname{div}\left(\left|\nabla \widehat{\phi}_{t}\right|^{2} \bar{m}\right) d t d \Omega \\
& -\int_{s}^{\tau} \int_{\Omega}\left[\frac{\widehat{\phi}_{t x}^{2} m_{1 x}}{2}+\frac{\widehat{\phi}_{t y}^{2} m_{2 y}}{2}\right] d t d \Omega-\int_{s}^{\tau} \int_{\Omega}\left[\widehat{\phi}_{t x} \widehat{\phi}_{t y} m_{2 x}+\widehat{\phi}_{t x} \widehat{\phi}_{t y} m_{1 y}\right] d t d \Omega \\
& +\int_{s}^{\tau} \int_{\Omega}\left[\frac{\widehat{\phi}_{t x}^{2} m_{2 y}}{2}+\frac{\widehat{\phi}_{t y}^{2} m_{1 x}}{2}\right] d t d \Omega \\
= & \left.\left(\nabla \widehat{\phi}_{t}, \nabla(\bar{m} \cdot \nabla \widehat{\phi})\right)_{L^{2}(\Omega)}\right|_{s} ^{\tau} \\
& +\int_{s}^{\tau} \int_{\Omega}\left[\frac{\widehat{\phi}_{t x}^{2} m_{2 y}}{2}+\frac{\widehat{\phi}_{t y}^{2} m_{1 x}}{2}-\frac{\widehat{\phi}_{t x}^{2} m_{1 x}}{2}-\frac{\widehat{\phi}_{t y}^{2} m_{2 y}}{2}\right] d t d \Omega \\
& -\int_{s}^{\tau} \int_{\Omega}\left[\widehat{\phi}_{t x} \widehat{\phi}_{t y} m_{2 x}+\widehat{\phi}_{t x} \widehat{\phi}_{t y} m_{1 y}\right] d t d \Omega,
\end{aligned}
$$

after again using the divergence theorem and the fact that $\int_{\Omega} \operatorname{div}\left(\left|\nabla \widehat{\phi}_{t}\right|^{2} \bar{m}\right) d \Omega=$ $\int_{\Gamma_{0}}\left|\nabla \widehat{\phi}_{t}\right|^{2} d \Gamma_{0}=0\left(\right.$ as $\left.\widehat{\phi}_{t}(t) \in H_{\Gamma_{0}}^{2}(\Omega)\right)$. 
(iii) To handle the biharmonic term, we use Green's theorem (1.9), the given boundary conditions of $(2.15),(4.14)$, and the fact that $\widehat{\phi} \in H_{\Gamma_{0}}^{2}(\Omega)$ to obtain

$$
\begin{aligned}
& \int_{s}^{\tau}\left(\Delta^{2} \widehat{\phi}, \bar{m} \cdot \nabla \widehat{\phi}\right)_{L^{2}(\Omega)} d t=\int_{s}^{\tau} a(\widehat{\phi}, \bar{m} \cdot \nabla \widehat{\phi}) d t \\
& +\alpha \int_{s}^{\tau} \int_{\Gamma_{1}} \widehat{\psi} \cdot \frac{\partial \bar{m} \cdot \nabla \widehat{\phi}}{\partial \nu} d \Gamma_{1} d t-\int_{s}^{\tau} \int_{\Gamma_{0}}\left(\Delta \widehat{\phi}+(1-\mu) B_{1} \widehat{\phi}\right) \frac{\partial^{2} \widehat{\phi}}{\partial \nu^{2}} d \Gamma_{0} d t .
\end{aligned}
$$

We note at this point that we can rewrite the first term on the right-hand side of (4.18) as

$$
\begin{aligned}
& \int_{s}^{\tau} a(\widehat{\phi}, \bar{m} \cdot \nabla \widehat{\phi}) d t \\
= & \frac{1}{2} \int_{s}^{\tau} \int_{\Omega} \bar{m} \cdot \nabla\left[\widehat{\phi}_{x x}^{2}+\widehat{\phi}_{y y}^{2}+2 \mu \widehat{\phi}_{x x} \widehat{\phi}_{y y}+2(1-\mu) \widehat{\phi}_{x y}^{2}\right] d t d \Omega \\
& +\mathcal{O}\left(\int_{0}^{T}\left\|\AA^{\frac{1}{2}} \widehat{\phi}\right\|_{L^{2}(\Omega)}^{2} d t\right),
\end{aligned}
$$

where $\mathcal{O}\left(\int_{0}^{T}\left\|\AA^{\frac{1}{2}} \widehat{\phi}\right\|_{L^{2}(\Omega)}^{2} d t\right)$ denotes a series of terms that can be majorized by the $L^{2}\left(0, T ; D\left(\AA^{\frac{1}{2}}\right)\right)$-norm of $\widehat{\phi}$. We consequently have by the divergence theorem that

$$
\begin{aligned}
& \int_{s}^{\tau} a(\widehat{\phi}, \bar{m} \cdot \nabla \widehat{\phi}) d t \\
= & \frac{1}{2} \int_{s}^{\tau} \int_{\Omega} \bar{m} \cdot \nabla\left[\widehat{\phi}_{x x}^{2}+\widehat{\phi}_{y y}^{2}+2 \mu \widehat{\phi}_{x x} \widehat{\phi}_{y y}+2(1-\mu) \widehat{\phi}_{x y}^{2}\right] d t d \Omega \\
& +\mathcal{O}\left(\int_{0}^{T}\left\|\AA^{\frac{1}{2}} \widehat{\phi}\right\|_{L^{2}(\Omega)}^{2} d t\right) \\
= & \frac{1}{2} \int_{s}^{\tau} \int_{\Omega}^{\tau} \operatorname{div}\left\{\bar{m}\left[\widehat{\phi}_{x x}^{2}+\widehat{\phi}_{y y}^{2}+2 \mu \widehat{\phi}_{x x} \widehat{\phi}_{y y}+2(1-\mu) \widehat{\phi}_{x y}^{2}\right]\right\} d t d \Omega \\
& +\mathcal{O}\left(\int_{0}^{T}\left\|\AA^{\frac{1}{2}} \widehat{\phi}\right\|_{L^{2}(\Omega)}^{2} d t\right) \\
= & \frac{1}{2} \int_{s}^{\tau} \int_{\Gamma_{0}}^{\tau}\left[\widehat{\phi}_{x x}^{2}+\widehat{\phi}_{y y}^{2}+2 \mu \widehat{\phi}_{x x} \widehat{\phi}_{y y}+2(1-\mu) \widehat{\phi}_{x y}^{2}\right] d t d \Gamma_{0} \\
& +\mathcal{O}\left(\int_{0}^{T}\left\|\AA^{\frac{1}{2}} \widehat{\phi}\right\|_{L^{2}(\Omega)}^{2} d t\right) \\
= & \frac{1}{2} \int_{s}^{\tau} \int_{\Gamma_{0}}(\Delta \widehat{\phi})^{2} d t+\mathcal{O}\left(\int_{0}^{T}\left\|\AA^{\frac{1}{2}} \widehat{\phi}\right\|_{L^{2}(\Omega)}^{2} d t\right),
\end{aligned}
$$

where in the last step above, we have used the fact (as reasoned in [11, Chapter 4]) that $\left.\widehat{\phi}\right|_{\Gamma_{0}}=\left.\frac{\partial \widehat{\phi}}{\partial \nu}\right|_{\Gamma_{0}}=0$ implies that $\widehat{\phi}_{x x}^{2}+\widehat{\phi}_{y y}^{2}+2 \mu \widehat{\phi}_{x x} \widehat{\phi}_{y y}+2(1-\mu) \widehat{\phi}_{x y}^{2}=(\Delta \widehat{\phi})^{2}$ on $\Gamma_{0}$. 
To handle the last term on the right-hand side of (4.18), we note that $B_{1} \widehat{\phi}=0$ on $\Gamma_{0}$, which implies that

$$
\Delta \widehat{\phi}=\Delta \widehat{\phi}+(1-\mu) B_{1} \widehat{\phi}=\frac{\partial^{2} \widehat{\phi}}{\partial \nu^{2}} \text { on } \Gamma_{0} .
$$

We consequently have upon the insertion of (4.20) into (4.18), followed by the consideration of (4.21) that

$$
\begin{aligned}
\int_{s}^{\tau} & \left(\Delta^{2} \widehat{\phi}, \bar{m} \cdot \nabla \widehat{\phi}\right)_{L^{2}(\Omega)} d t=-\frac{1}{2} \int_{s}^{\tau}\|\Delta \widehat{\phi}\|_{L^{2}\left(\Gamma_{0}\right)}^{2} d t \\
& +\alpha \int_{s}^{\tau} \int_{\Gamma_{1}} \widehat{\psi} \cdot \frac{\partial \bar{m} \cdot \nabla \widehat{\phi}}{\partial \nu} d \Gamma_{1} d t+\mathcal{O}\left(\int_{0}^{T}\left\|\AA^{\frac{1}{2}} \widehat{\phi}\right\|_{L^{2}(\Omega)}^{2} d t\right)
\end{aligned}
$$

(iv) To handle the right-hand side of (4.15), an integration by parts yields

$$
\begin{aligned}
& \int_{s}^{\tau}\left(c_{0} \widehat{\psi}+c_{1} \widehat{\psi}_{t}+c_{2} \widehat{\phi}+c_{3} \widehat{\phi}_{t}+c_{4} \Delta \widehat{\phi}, \bar{m} \cdot \nabla \widehat{\phi}\right)_{L^{2}(\Omega)} d t \\
= & c_{1}\left[(\widehat{\psi}, \bar{m} \cdot \nabla \widehat{\phi})_{L^{2}(\Omega)}\right]_{s}^{\tau}-c_{1} \int_{s}^{\tau}\left(\widehat{\psi}, \bar{m} \cdot \nabla \widehat{\phi}_{t}\right)_{L^{2}(\Omega)} d t \\
& +\int_{s}^{\tau}\left(c_{0} \widehat{\psi}+c_{2} \widehat{\phi}+c_{3} \widehat{\phi}_{t}+c_{4} \Delta \widehat{\phi}, \bar{m} \cdot \nabla \widehat{\phi}\right)_{L^{2}(\Omega)} d t .
\end{aligned}
$$

As $\bar{m} \cdot \nabla \widehat{\phi} \in C\left([0, T] ; H_{\Gamma_{0}, \gamma}^{1}(\Omega)\right)$, we have for all $t \in[0, T]$,

$$
(\widehat{\psi}(t), \bar{m} \cdot \nabla \widehat{\phi}(t))_{L^{2}(\Omega)}=\langle\widehat{\psi}(t), \bar{m} \cdot \nabla \widehat{\phi}(t)\rangle_{H_{\Gamma_{0}, \gamma}^{-1}(\Omega) \times H_{\Gamma_{0}, \gamma}^{1}(\Omega)} .
$$

Accordingly, we have

$$
\begin{aligned}
& \int_{s}^{\tau}\left(c_{0} \widehat{\psi}+c_{1} \widehat{\psi}_{t}+c_{2} \widehat{\phi}+c_{3} \widehat{\phi}_{t}+c_{4} \Delta \widehat{\phi}, \bar{m} \cdot \nabla \widehat{\phi}\right)_{L^{2}(\Omega)} d t \\
& \quad=c_{1}\left[\langle\widehat{\psi}, \bar{m} \cdot \nabla \widehat{\phi}\rangle_{H_{\Gamma_{0}, \gamma}^{-1}(\Omega) \times H_{\Gamma_{0}, \gamma}^{1}(\Omega)}^{\tau}\right]_{s} \\
& -c_{1} \int_{s}^{\tau}\left(\widehat{\psi}, \bar{m} \cdot \nabla \widehat{\phi}_{t}\right)_{L^{2}(\Omega)} d t+\int_{s}^{\tau}\left(c_{0} \widehat{\psi}+c_{2} \widehat{\phi}+c_{3} \widehat{\phi}_{t}+c_{4} \Delta \widehat{\phi}, \bar{m} \cdot \nabla \widehat{\phi}\right)_{L^{2}(\Omega)} d t .
\end{aligned}
$$

To finish the proof, we rewrite (4.15) by collecting the relations given above in (4.16), (4.17), (4.22), and (4.23) to attain

$$
\begin{aligned}
& \frac{1}{2} \int_{s}^{\tau}\|\Delta \widehat{\phi}\|_{L^{2}\left(\Gamma_{0}\right)}^{2} d t=\alpha \int_{s}^{\tau} \int_{\Gamma_{1}} \widehat{\psi} \cdot \frac{\partial \bar{m} \cdot \nabla \widehat{\phi}}{\partial \nu} d \Gamma_{1} d t \\
& +\mathcal{O}\left(\int_{0}^{T}\left\|\AA^{\frac{1}{2}} \widehat{\phi}\right\|_{L^{2}(\Omega)}^{2} d t\right)+\frac{1}{2} \int_{s}^{\tau} \int_{\Omega} \widehat{\phi}_{t}^{2}\left[m_{1 x}+m_{2 y}\right] d t d \Omega
\end{aligned}
$$




$$
\begin{aligned}
& -\int_{s}^{\tau}\left(c_{0} \widehat{\psi}+c_{2} \widehat{\phi}+c_{3} \widehat{\phi}_{t}+c_{4} \Delta \widehat{\phi}, \bar{m} \cdot \nabla \widehat{\phi}\right)_{L^{2}(\Omega)} d t \\
& +\gamma \int_{s}^{\tau} \int_{\Omega}\left[\frac{\widehat{\phi}_{t x}^{2} m_{2 y}}{2}+\frac{\widehat{\phi}_{t y}^{2} m_{1 x}}{2}-\frac{\widehat{\phi}_{t x}^{2} m_{1 x}}{2}-\frac{\widehat{\phi}_{t y}^{2} m_{2 y}}{2}\right] d t d \Omega \\
& -\gamma \int_{s}^{\tau} \int_{\Omega}\left[\widehat{\phi}_{t x} \widehat{\phi}_{t y} m_{2 x}+\widehat{\phi}_{t x} \widehat{\phi}_{t y} m_{1 y}\right] d t d \Omega+c_{1} \int_{s}^{\tau}\left(\widehat{\psi}, \bar{m} \cdot \nabla \widehat{\phi}_{t}\right)_{L^{2}(\Omega)} d t \\
& +\left[\left(\widehat{\phi}_{t}, \bar{m} \cdot \nabla \widehat{\phi}\right)_{L^{2}(\Omega)}+\gamma\left(\nabla \widehat{\phi}_{t}, \nabla(\bar{m} \cdot \nabla \widehat{\phi})\right)_{L^{2}(\Omega)}-c_{1}\langle\widehat{\psi}, \bar{m} \cdot \nabla \widehat{\phi}\rangle_{H_{\Gamma_{0}, \gamma}^{-1}(\Omega) \times H_{\Gamma_{0}, \gamma}^{1}(\Omega)}\right]_{s}^{\tau} .
\end{aligned}
$$

The desired inequality (4.13) now comes about by majorizing the right-hand side of this expression (note that in this majorization we are using implicitly the fact that $\left.\frac{\partial \bar{m} \cdot \nabla \widehat{\phi}}{\partial \nu}\right|_{\Gamma_{1}}$ is a "lower order term," as $\left.\left.\bar{m}\right|_{\Gamma_{1}}=0\right)$.

Proposition 4.6. With the vector field $\bar{h}$ as defined in (1.3), the solution $\left[\widehat{\phi}, \widehat{\phi}_{t}, \widehat{\psi}\right]$ to $(2.15)$, corresponding to terminal data $\left[\phi_{0}, \phi_{1}, \psi_{0}\right] \in D\left(\left[\mathcal{A}_{\gamma}^{*}\right]^{2}\right)$, satisfies equality (2.36) for arbitrary $\epsilon_{0} \in[0, T)$.

Proof. We multiply (2.15) by $\bar{h} \cdot \nabla \widehat{\phi}-\frac{1}{2} \widehat{\phi}$ and subsequently integrate in time and space; i.e., we will consider the equation

$$
\int_{\epsilon_{0}}^{T-\epsilon_{0}}\left(\widehat{\phi}_{t t}-\gamma \Delta \widehat{\phi}_{t t}+\Delta^{2} \widehat{\phi}-\left[c_{0} \widehat{\psi}+c_{1} \widehat{\psi}_{t}+c_{2} \widehat{\phi}+c_{3} \widehat{\phi}_{t}\right], \bar{h} \cdot \nabla \widehat{\phi}-\frac{1}{2} \widehat{\phi}\right)_{L^{2}(\Omega)} d t=0 .
$$

First, using directly the computations performed in [12] for the quantity $\int_{\epsilon_{0}}^{T-\epsilon_{0}}\left(\widehat{\phi}_{t t}-\right.$ $\left.\gamma \Delta \widehat{\phi}_{t t}+\Delta^{2} \widehat{\phi}, \bar{h} \cdot \nabla \widehat{\phi}-\frac{1}{2} \widehat{\phi}\right)_{L^{2}(\Omega)} d t$, in the case that $\bar{h}$ is a radial vector field (see the relations (3.12) and (3.16) of [12]), we have

$$
\begin{aligned}
& \int_{\epsilon_{0}}^{T-\epsilon_{0}}\left(\widehat{\phi}_{t t}-\gamma \Delta \widehat{\phi}_{t t}+\Delta^{2} \phi, \bar{h} \cdot \nabla \widehat{\phi}-\frac{1}{2} \widehat{\phi}\right)_{L^{2}(\Omega)} d t \\
= & {\left[(\widehat{\phi}, \bar{h} \cdot \nabla \widehat{\phi})_{L^{2}(\Omega)}+\gamma\left(\nabla \widehat{\phi}_{t}, \nabla(\bar{h} \cdot \nabla \widehat{\phi})\right)_{L^{2}(\Omega)}-\frac{1}{2}\left(\widehat{\phi}_{t}, \widehat{\phi}\right)_{L^{2}(\Omega)}\right]_{t=\epsilon_{0}}^{t=T-\epsilon_{0}} } \\
- & {\left[\frac{\gamma}{2}\left(\nabla \widehat{\phi}_{t}, \nabla \widehat{\phi}\right)_{L^{2}(\Omega)}\right]_{t=\epsilon_{0}}^{t=T-\epsilon_{0}}-\frac{1}{2} \int_{\epsilon_{0}}^{T-\epsilon_{0}} \int_{\Gamma_{1}} \bar{h} \cdot \nu\left(\widehat{\phi}_{t}^{2}+\gamma\left|\nabla \widehat{\phi}_{t}\right|^{2}\right) d \Gamma d t } \\
+ & \int_{\epsilon_{0}}^{T-\epsilon_{0}}\left[\frac{1}{2}\left\|P_{\gamma}^{\frac{1}{2}} \widehat{\phi}_{t}\right\|_{L^{2}(\Omega)}^{2}+\left\|\widehat{\phi}_{t}\right\|_{L^{2}(\Omega)}^{2}-\gamma\left(\bar{h} \cdot \nabla \widehat{\phi}-\frac{1}{2} \widehat{\phi}, \frac{\partial \widehat{\phi}_{t t}}{\partial \nu}\right)_{L^{2}\left(\Gamma_{1}\right)}\right] d t \\
+ & \int_{\epsilon_{0}}^{T-\epsilon_{0}}\left[\frac{1}{2}\left\|\AA^{\frac{1}{2}} \widehat{\phi}\right\|^{2}+\frac{1}{2} \int_{\Gamma_{0}} \bar{h} \cdot \nu(\Delta \widehat{\phi})^{2} d \Gamma-\left(\Delta \widehat{\phi}, \frac{\partial}{\partial \nu}(\bar{h} \cdot \nabla \widehat{\phi})\right)_{L^{2}\left(\Gamma_{0}\right)}\right] d t \\
+ & \int_{\epsilon_{0}}^{T-\epsilon_{0}}\left[\left(\frac{\partial \Delta \widehat{\phi}}{\partial \nu}+(1-\mu) \frac{\partial B_{2} \widehat{\phi}}{\partial \tau}, \bar{h} \cdot \nabla \widehat{\phi}-\frac{1}{2} \widehat{\phi}\right)_{L^{2}\left(\Gamma_{1}\right)}\right] d t \\
& \left.-\left(\Delta \widehat{\phi}+(1-\mu) B_{1} \widehat{\phi}, \frac{\partial}{\partial \nu}\left(\bar{h} \cdot \nabla \widehat{\phi}-\frac{1}{2} \widehat{\phi}\right)\right)_{L^{2}\left(\Gamma_{1}\right)}\right] d t
\end{aligned}
$$


382

$$
\begin{gathered}
+\int_{\epsilon_{0}}^{T-\epsilon_{0}} \int_{\Gamma_{1}} \frac{\bar{h} \cdot \nu}{2}\left[\left(\frac{\partial^{2} \widehat{\phi}}{\partial x^{2}}\right)^{2}+\left(\frac{\partial^{2} \widehat{\phi}}{\partial y^{2}}\right)^{2}+2 \mu\left(\frac{\partial^{2} \widehat{\phi}}{\partial x^{2}}\right)\left(\frac{\partial^{2} \widehat{\phi}}{\partial y^{2}}\right)\right. \\
\left.+2(1-\mu)\left(\frac{\partial^{2} \widehat{\phi}}{\partial x \partial y}\right)^{2}\right] d t d \Gamma .
\end{gathered}
$$

Using the boundary conditions in (2.15) and the fact that $\left.\frac{\partial(\bar{h} \cdot \nabla \phi)}{\partial \nu}\right|_{\Gamma_{0}}=$ $\left.(\bar{h} \cdot \nu) \Delta \phi\right|_{\Gamma_{0}}$, this equation becomes

$$
\begin{gathered}
\int_{\epsilon_{0}}^{T-\epsilon_{0}}\left(\widehat{\phi}_{t t}-\gamma \Delta \widehat{\phi}_{t t}+\Delta^{2} \phi, \bar{h} \cdot \nabla \widehat{\phi}-\frac{1}{2} \widehat{\phi}\right)_{L^{2}(\Omega)} d t=\frac{1}{2} \int_{\epsilon_{0}}^{T-\epsilon_{0}} E_{\widehat{\phi}}(t) d t \\
+\left[\left(\widehat{\phi}_{t}, \bar{h} \cdot \nabla \widehat{\phi}\right)_{L^{2}(\Omega)}+\gamma\left(\nabla \widehat{\phi}_{t}, \nabla(\bar{h} \cdot \nabla \widehat{\phi})\right)_{L^{2}(\Omega)}-\frac{1}{2}\left(\widehat{\phi}_{t}, \widehat{\phi}\right)_{L^{2}(\Omega)}\right]_{t=\epsilon_{0}}^{t=T-\epsilon_{0}} \\
-\left[\frac{\gamma}{2}\left(\nabla \widehat{\phi}_{t}, \nabla \widehat{\phi}\right)_{L^{2}(\Omega)}\right]_{t=\epsilon_{0}}^{t=T-\epsilon_{0}}-\frac{1}{2} \int_{\epsilon_{0}}^{T-\epsilon_{0}} \int_{\Gamma_{1}} \bar{h} \cdot \nu\left(\widehat{\phi}_{t}^{2}+\gamma\left|\nabla \widehat{\phi}_{t}\right|^{2}\right) d \Gamma d t \\
+\int_{\epsilon_{0}}^{T-\epsilon_{0}}\left[\left\|\widehat{\phi}_{t}\right\|_{L^{2}(\Omega)}^{2}-\frac{1}{2} \int_{\Gamma_{0}} \bar{h} \cdot \nu(\Delta \widehat{\phi})^{2} d \Gamma\right] d t \\
+\int_{\epsilon_{0}}^{T-\epsilon_{0}}\left[\alpha\left(\widehat{\psi}, \frac{\partial}{\partial \nu}\left(\bar{h} \cdot \nabla \widehat{\phi}-\frac{1}{2} \widehat{\phi}\right)\right)_{L^{2}\left(\Gamma_{1}\right)}\right. \\
\left.+\left(\gamma \frac{\partial}{\partial \nu}\left(\xi^{2} \widehat{\phi}+2 \xi \widehat{\phi}_{t}\right)-\alpha \frac{\partial \hat{\psi}}{\partial \nu}, \bar{h} \cdot \nabla \widehat{\phi}-\frac{1}{2} \widehat{\phi}\right){ }_{L^{2}\left(\Gamma_{1}\right)}\right] d t \\
+\int_{\epsilon_{0}}^{T-\epsilon_{0}} \int_{\Gamma_{1}} \frac{\bar{h} \cdot \nu}{2}\left[\left(\frac{\partial^{2} \widehat{\phi}}{\partial x^{2}}\right)^{2}+\left(\frac{\partial^{2} \widehat{\phi}}{\partial y^{2}}\right)^{2}+2 \mu\left(\frac{\partial^{2} \widehat{\phi}}{\partial x^{2}}\right)\left(\frac{\partial^{2} \widehat{\phi}}{\partial y^{2}}\right)\right. \\
\left.+2(1-\mu)\left(\frac{\partial^{2} \widehat{\phi}}{\partial x \partial y}\right)^{2}\right] d t d \Gamma .
\end{gathered}
$$

Second, we multiply $\left[c_{0} \widehat{\psi}+c_{1} \widehat{\psi}_{t}+c_{2} \widehat{\phi}+c_{3} \widehat{\phi}_{t}+c_{4} \Delta \widehat{\phi}\right]$ by $\bar{h} \cdot \nabla \widehat{\phi}-\frac{1}{2} \widehat{\phi}$ and integrate by parts to obtain

$$
\begin{aligned}
& \int_{\epsilon_{0}}^{T-\epsilon_{0}}\left(c_{0} \widehat{\psi}+c_{1} \widehat{\psi}_{t}+c_{2} \widehat{\phi}+c_{3} \widehat{\phi}_{t}+c_{4} \Delta \widehat{\phi}, \bar{h} \cdot \nabla \widehat{\phi}-\frac{1}{2} \widehat{\phi}\right)_{L^{2}(\Omega)} d t \\
= & c_{1}\left[\left(\widehat{\psi}, \bar{h} \cdot \nabla \widehat{\phi}-\frac{1}{2} \widehat{\phi}\right)_{L^{2}(\Omega)}\right]_{t=\epsilon_{0}}^{t=T-\epsilon_{0}} \\
& -c_{1} \int_{\epsilon_{0}}^{T-\epsilon_{0}}\left(\widehat{\psi}, \bar{h} \cdot \nabla \widehat{\phi}_{t}-\frac{1}{2} \widehat{\phi}_{t}\right)_{L^{2}(\Omega)} d t
\end{aligned}
$$




$$
+\int_{\epsilon_{0}}^{T-\epsilon_{0}}\left(c_{0} \widehat{\psi}+c_{2} \widehat{\phi}+c_{3} \widehat{\phi}_{t}+c_{4} \Delta \widehat{\phi}, \bar{h} \cdot \nabla \widehat{\phi}-\frac{1}{2} \widehat{\phi}\right)_{L^{2}(\Omega)} d t
$$

To now obtain (2.36), we combine the expressions (4.25) and (4.27)-(4.28) and follow this by a rearrangement of terms.

\section{REFERENCES}

[1] J. P. Aubin, Analyse fonctionelle appliquée, Tome 2, Presses Universitaire de France, Paris, 1979.

[2] G. Avalos, The exponential stability of a coupled hyperbolic/parabolic system arising in structural acoustics, Abstr. Appl. Anal., 1 (1996), pp. 203-217.

[3] G. Avalos And I. Lasiecka, Exponential stability of a thermoelastic system with free boundary conditions without mechanical dissipation, SIAM J. Math. Anal., 29 (1998), pp. 155-182.

[4] G. Avalos AND I. LasieCKa, Exponential stability of a thermoelastic system without mechanical dissipation, Rend. Instit. Mat. Univ. Trieste, 28 (1997), pp. 1-28.

[5] G. Avalos, Exact controllability of a thermoelastic system with control in the thermal component only, Differential Integral Equations, to appear.

[6] L. De Teresa and E. Zuazua, Controllability for the linear system of thermoelastic plates, Adv. Differential Equations, 1 (1996), pp. 369-402.

[7] P. Grisvard, Caracterization de quelques espaces d'interpolation, Arch. Rational Mech. Anal., 25 (1967), pp. 40-63.

[8] S. Hansen and B. Zhang, Boundary control of a linear thermoelastic beam, J. Math. Anal. Appl., 210 (1997), pp. 182-205.

[9] V. Hutson And J. S. Pym, Applications of Functional Analysis and Operator Theory, Academic Press, New York, 1980.

[10] V. Isakov, On the uniqueness of the continuation for a thermoelasticity system, Differential Equations, to appear.

[11] J. E. LAGnese, Boundary Stabilization of Thin Plates, SIAM Stud. Appl. Math. 10, SIAM, Philadelphia, 1989.

[12] J. LAGnese, The reachability problem for thermoelastic plates, Arch. Rational Mech. Anal., 112 (1990), pp. 223-267.

[13] I. Lasiecka AND R. TRIGgiani, Uniform stabilization of the wave equation with Dirichlet or Neumann feedback control without geometrical conditions, Appl. Math. Optim., 25 (1992), pp. 189-224.

[14] I. Lasiecka And R. Triggiani, Exact controllability of the wave equation with Neumann boundary control, Appl. Math. Optim., 19 (1989), pp. 243-290.

[15] I. Lasiecka And R. TRiggiani, Sharp trace estimates of solutions to Kirchoff and EulerBernoulli equations, Appl. Math. Optim., 28 (1993), pp. 277-306.

[16] I. Lasiecka And R. Triggiani, Structural decomposition of thermoelastic semigroups with rotational forces, Semigroup Forum, 5 (1999), pp. 585-599.

[17] I. Lasiecka and R. Triggiani, Sharp regularity theory for elastic and thermo-elastic Kirchoff equations with free boundary conditions, Rocky Mountain J. Math., to appear.

[18] I. Lasiecka And R. TRigGiani, Analyticity of thermo-elastic semigroups with free boundary conditions, Ann. Scuola Norm. Sup. Pisa Cl. Sci. (3-4), 27 (1998), pp. 457-482.

[19] G. Lebeau and E. Zuazua, Null-controllability of a system of linear thermoelasticity, Arch. Rational Mech. Anal., 141 (1998), pp. 297-329.

[20] G. Leugering A decomposition method for integro-partial differential equations and applications, J. Math. Pures Appl., 71 (1992), pp. 561-587.

[21] J. L. Lions And E. Magenes, Non-Homogeneous Boundary Value Problems and Applications, vol. 1., Springer-Verlag, New York, 1972.

[22] W. Liu, Partial exact controllability and exponential stability in higher-dimensional linear thermoelasticity, ESAIM Control Optim. Calc. Var., 3 (1998), pp. 23-48.

[23] W. Liu, Erratum on partial exact controllability and exponential stability in higher-dimensional linear thermoelasticity, ESAIM Control Optim. Calc. Var., 3 (1998), pp. 323-328.

[24] J. Simon, Compact sets in the space $L^{p}(0, T ; B)$, Ann. Mat. Pura Appl. (4), 146 (1987), pp. 6596. 